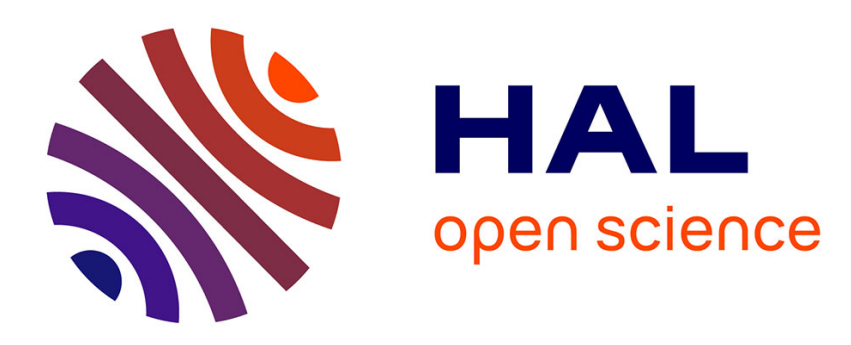

\title{
A new brittle-elastoviscoplastic fluid based on the Drucker-Prager plasticity
}

Pierre Saramito

\section{To cite this version:}

Pierre Saramito. A new brittle-elastoviscoplastic fluid based on the Drucker-Prager plasticity. Journal of Non-Newtonian Fluid Mechanics, 2021, 294, pp.104584. 10.1016/j.jnnfm.2021.104584 . hal03257512

\section{HAL Id: hal-03257512 \\ https://hal.science/hal-03257512}

Submitted on 11 Jun 2021

HAL is a multi-disciplinary open access archive for the deposit and dissemination of scientific research documents, whether they are published or not. The documents may come from teaching and research institutions in France or abroad, or from public or private research centers.
L'archive ouverte pluridisciplinaire HAL, est destinée au dépôt et à la diffusion de documents scientifiques de niveau recherche, publiés ou non, émanant des établissements d'enseignement et de recherche français ou étrangers, des laboratoires publics ou privés. 


\title{
A new brittle-elastoviscoplastic fluid based on the Drucker-Prager plasticity
}

\author{
Pierre Saramito ${ }^{a, *}$ \\ ${ }^{a}$ Lab. Jean Kuntzmann-CNRS and Université Grenoble-Alpes, F-38041 Grenoble, France
}

\begin{abstract}
A new brittle-elastoviscoplastic (BEVP) fluid model is presented in this paper. This model is relatively simple to use, as it contains few material parameters and a simple fixed-point algorithm is effective for solving the coupled system of equations. The model combines some existing fundamental features such as elasticity, plasticity and brittle damage. The combination of them is based on thermodynamics that ensures the positivity of the dissipation and the Onsager symmetry. Moreover, thermodynamics allows to point out the link between thixotropy and damage in the context of elastoviscoplastic (EVP) fluids. Theoretical results on the Drucker-Prager plasticity criterion are completed in order to use it here. Preliminary results with the proposed BEVP model are very encouraging: it is able to represent the pre-failure, failure and post-failure behavior of quasi-brittle materials.
\end{abstract}

Keywords: elastoviscoplastic fluid, damage, thixotropy, finite element method

\section{Introduction}

Materials that present microstructure of particles with frictional contacts and a large scale rearrangements are very common in nature. Typical examples are dry or wet granular flows and concentrated suspensions. Also, rocks and cements undergoing large and unbounded deformations share this microstructural aspect. At a larger scale, the dynamic of earthquakes and the sea ice, for predicting the climate evolution, are potential applications of the present work. The development of efficient rheological models for the flow of dense granular maters and suspensions is also a challenge for continuous models, where discrete simulations are still more relevant for applications, but limited in terms of the particle number. The most popular continuous description of granular flows is certainly the viscoplastic $\mu(I)$ rheology [31], despite its mathematical issues [3, 54]. The $\mu(I)$ pressure-dependent yield stress feature was recently revisited by Daviet and Bertails [11, 10], based on the elegant de Saxcé mathematical formalization [27] of the Drucker-Prager plasticity criterion [14]. The present paper is a contribution in this direction, in order to develop continuous models that take into account microstructural frictional contacts and large scale rearrangements. For that purpose, theoretical results on the Drucker-Prager plasticity criterion are completed in order to use it for general elastoviscoplastic (EVP) fluids.

During the flow, the properties of these materials are likely to develop. Indeed, large stresses tend to break grains. For instance, during the sea ice flow, the floes are broken, as shown on Fig. 1.left, but a healing process due to freezing tends to collapse neighbors floes with a time scale of few days [9]. A similar effect is observed with earthquakes, where faults could slowly heal. Developed in the context of elastoplastic solids, the damage theory $[36,44]$ is widely used from years, with

\footnotetext{
* Corresponding author

Email address: Pierre.Saramito@imag.fr (Pierre Saramito)
} 

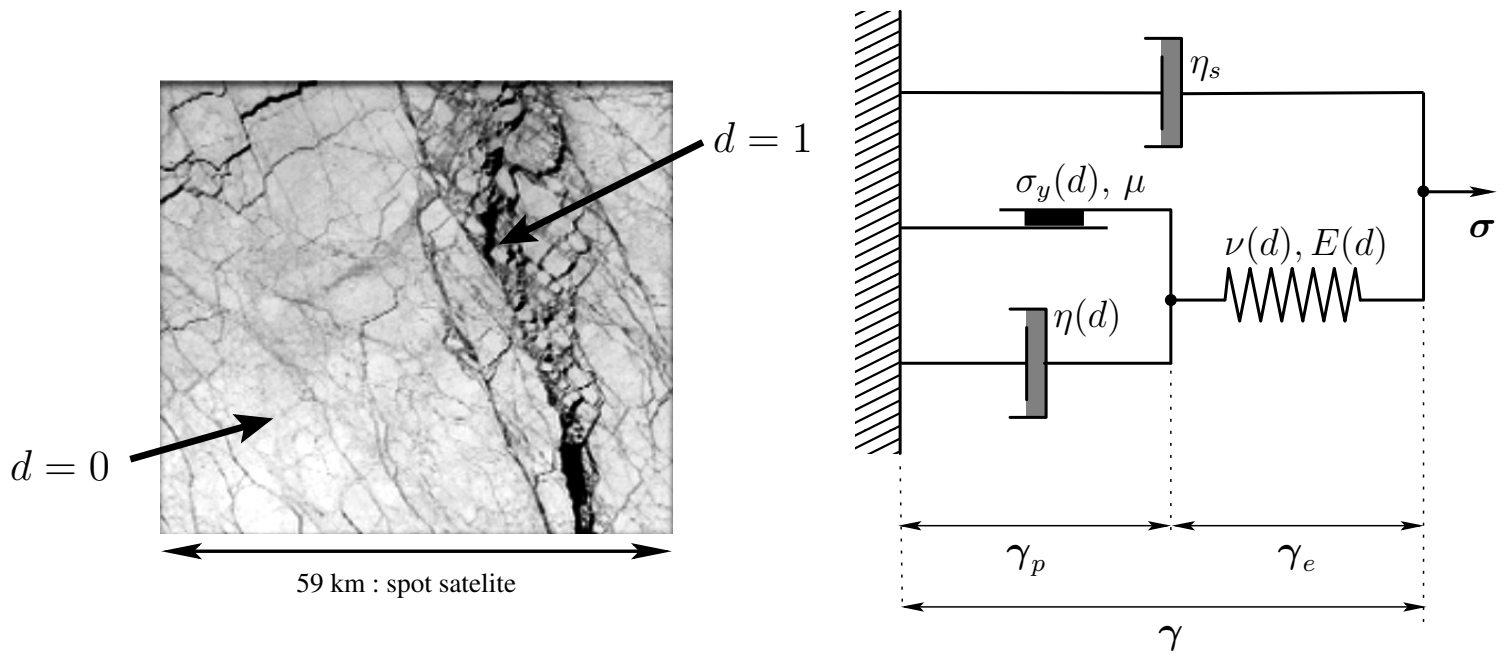

Figure 1: (left) The damage variable $d \in[0,1]$ is defined by Kachanov [33] as the density of micro-cracks and comminution of the fractured material at the macroscopic level. The background image is a SPOT satellite aerial picture of a $59 \times 59 \mathrm{~km}^{2}$ portion of the Arctic sea ice cover centered around $80.18^{\circ} \mathrm{N}, 108.55^{\circ} \mathrm{W}$. (right) The rheological model combines two viscous elements $\eta(d)$ and $\eta_{s}$ with a dry-friction element including both yield stress and Coulomb friction $\mu$, together with a compressible elastic element with a Poisson ratio $\nu(d)$.

applications to rocks, concretes and steels. Damage mechanics for modeling strongly elastic, but not brittle, solid materials such as bread dough was also used by Phan-Thien et al. [46]. Conversely, thixotropy $[4,42,35]$ is a different kind of approach, used mostly in the context of viscoelastic fluids, such as suspensions of colloidal and non-colloidal particles that form flocculated systems, as well as gels that form cross-linked systems. Such materials are handled in the food, petroleum and cosmetic industries. One of the main characteristics of both damage and thixotropic effects is the time-dependent change in the material parameters associated to elasticity, viscosity and plasticity.

Thus, the proposed brittle-elastoviscoplastic (BEVP) model, represented on Fig. 1.right, shares some structural similarities with some existing thixo-elastoviscoplastic models (TEVP, see e.g. $[35,13,12])$. By introducing an abstract mathematical model suitable for such soft-solids and complex fluids, the present paper points out these similarities. Using this abstract model, three main features, namely elasticity, plasticity and damage could be combined together, as a Lego game, with the fundamental viscous effects. This abstraction is first illustrated with the BMP [5] thixotropic model before to develop the present BEVP model. Our abstract framework bases on the thermodynamics with internal variables and a potential of dissipation, known as the generalized standard materials (GSM). GSM was introduced by Halphen and Nguyen [23] in the context of elastoplasticity of solids and previously used by the present author for the development of elastoviscoplastic (EVP) fluids [49, 50]. While thermodynamics is popular in the elastoplastic solid's community, observe that its usage is much less advanced in the complex fluid's community. Remarkable contributions are those of Leonov [38], which was based on thermodynamics to propose a viscoelastic fluid and Beris and Edwards [6], who proposed the Poisson bracket tool for the development of new fluid models. Thus, to the author's best knowledge, the present theoretical approach is new in the context of these TEVP and BEVP complex fluids.

The outline of the paper is as follows: section 1 presents the thermodynamic framework that leads to the mathematical expression of the rheological model. At this stage, the obtained model still contains three black-boxes, namely the elasticity, the plasticity and the damage, that will be chosen in section 2 for obtaining the new BEVP model. Section 3 presents preliminary results on the uniaxial compression benchmark. The paper contains two appendices. The first one is the complete and self-contained proof of a theoretical result on the Drucker-Prager plasticity criterion 
in order to use it for general EVP fluids. The second one is the details of the numerical method used in section 3 .

\section{Abstract problem statement}

In this section, the thermodynamic framework is defined. First, the free energy and the potential of dissipation are introduced. Next, constitutive equations are obtained by differentiation of these two previous fundamental functions. A thorough discussion about the dissipation is developed before to expand the abstract problem statement. This section closes with a practical example: the thixotropic BMP model and its dissipative effects are discussed. Recall that the abstract mathematical model still contains three black-boxes, namely the elasticity, the plasticity and the damage, that will be chosen in a forthcoming section for obtaining the new BEVP model.

The impatient reader - and the reader who is unfamiliar with the thermodynamic framework could jump directly to section 1.4 where the complete set of equations governing such a flow is presented, before reading section 1.5 where the BMP model is discussed.

\subsection{Thermodynamic framework}

\begin{tabular}{|r|l|}
\hline \multicolumn{1}{|c|}{ Notation } & Description \\
\hline \hline$N$ & dimension of the physical space \\
\hline $\mathbb{R}_{s}^{N \times N}$ & set of real symmetric $N \times N$ matrix \\
\hline $\boldsymbol{I}$ & identity tensor \\
\hline \hline $\operatorname{tr} \boldsymbol{\tau}, \mathbf{d e v} \boldsymbol{\tau}$ & trace and deviator of a tensor $\boldsymbol{\tau}$ \\
\hline$d$ & damage (scalar) \\
\hline$Y$ & strain energy release rate (scalar) \\
\hline $\boldsymbol{u}$ & velocity \\
\hline $\boldsymbol{\gamma}$ & total deformation \\
\hline$\gamma_{e}$ & elastic deformation \\
\hline$\gamma_{p}$ & plastic deformation \\
\hline $\boldsymbol{\sigma}$ & Cauchy total stress \\
\hline $\boldsymbol{\sigma}_{e}$ & elastic stress \\
\hline$W e$ & Weissenberg number, viscoelasticity \\
\hline$\gamma_{y}$ & elastoplastic yield deformation \\
\hline$W e_{d}$ & for damage relaxation \\
\hline$\gamma_{c}$ & elastodamage yield deformation \\
\hline
\end{tabular}

\begin{tabular}{|r|l|}
\hline \multicolumn{1}{|l|}{ Notation } & Description \\
\hline \hline$\rho$ & density \\
\hline$\eta_{0}, \eta_{s}$ & viscosities \\
\hline$\eta_{t o t}$ & $=\eta_{0}+\eta_{s}$, total viscosity \\
\hline$\eta_{d}$ & damage-related viscosity \\
\hline$\sigma_{y}$ & viscoplastic cohesion (yield stress) \\
\hline$\sigma_{c}$ & damage cohesion \\
\hline$\mu$ & friction coefficient (dimensionless) \\
\hline$\nu, E$ & Poisson ratio and elastic modulus \\
\hline$G, \lambda$ & Lamé coefficients \\
\hline $\mathbb{A}$ & elasticity fourth order operator \\
\hline$\psi \psi$ & Helmholtz free energy \\
\hline$\phi$ & dissipation potential \\
\hline$\phi_{p}$ & viscoplastic dissipation potential \\
\hline$\phi_{d}$ & damage dissipation potential \\
\hline$w$ & total dissipation \\
\hline$w_{p}$ & viscoplastic dissipation \\
\hline$w_{d}$ & damage dissipation \\
\hline$K_{\mu}$ & Drucker-Prager cone \\
\hline$T_{\mu, \sigma_{y}}$ & translated Drucker-Prager cone \\
\hline $\mathscr{I}_{C}$ & indicator function of the set $C$ \\
\hline & \\
\hline & \\
\hline & \\
\hline & \\
\hline &
\end{tabular}

Table 1: Table of notations.

The total deformation tensor $\gamma$ is assumed to split as the sum of $\gamma_{e}$, the elastic deformation, and $\gamma_{p}$, its complement:

$$
\gamma=\gamma_{p}+\gamma_{e}
$$

Following Kachanov [33], let us introduce the progressive damage variable $d$ : it quantifies the density of micro-cracks and comminution of the fractured material at the macroscopic level (see Fig. 1.left). It evolves between $d=0$ for an undamaged and $d=1$ for a completely damaged material. As in most previous progressive damage models $[33,55,57,2]$, and based on the notion of effective stress, we choose to let the elasticity operator $\mathbb{A}(d)$ of the material vary with the level of damage. 
The thermodynamic framework of standard generalized materials [23] (see also [51, p. 222]) is considered here. Let $\left(\gamma, \gamma_{p}, d\right)$ be the three independent thermodynamic state variables of our material. At any time, we assume that we are able to impose some arbitrarily value to the rate variables $\left(\dot{\gamma}, \dot{\gamma}_{p}, \dot{d}\right)$ without changing the values of the state variables $\left(\boldsymbol{\gamma}, \boldsymbol{\gamma}_{p}, d\right)$, so state variables and rate variables are considered as independent thermodynamic variables. The specific Helmholtz free energy $\psi$ and the dissipation potential $\phi$ are defined by

$$
\begin{aligned}
\psi\left(\boldsymbol{\gamma}, \boldsymbol{\gamma}_{p}, d\right) & =\frac{1}{2 \rho}\left|\boldsymbol{\gamma}-\boldsymbol{\gamma}_{p}\right|_{\mathbb{A}(d)}^{2} \\
\phi\left(\left[\boldsymbol{\gamma}, \boldsymbol{\gamma}_{p}, d\right] ; \dot{\gamma}, \dot{\gamma}_{p}, \dot{d}\right) & =\mathscr{I}_{\operatorname{ker}(\operatorname{tr})}(\dot{\gamma})+\eta_{s}|\dot{\boldsymbol{\gamma}}|^{2}+\phi_{p}\left([d] ; \dot{\gamma}_{p}\right)+\phi_{d}\left(\left[\gamma-\gamma_{p}, d\right] ; \dot{d}\right)
\end{aligned}
$$

In $(2 \mathrm{a})$, the density of the material is denoted by $\rho$ and is assumed to be constant. The notations used all along the paper are summarized in Table 1. Let us denote by $|\boldsymbol{\delta}|$ the tensor norm: $|\boldsymbol{\delta}|^{2}=\boldsymbol{\delta}: \boldsymbol{\delta}$, for any $\boldsymbol{\delta} \in \mathbb{R}_{s}^{N \times N}$, where $N \geqslant 1$ is the physical space dimension, and $\mathbb{R}_{s}^{N \times N}$ the space of symmetric $N \times N$ real matrix. For any matrix $\boldsymbol{\delta}, \boldsymbol{\tau} \in \mathbb{R}_{s}^{N \times N}$, the associated dot product is $\boldsymbol{\delta}: \boldsymbol{\tau}=\sum_{i, j=1}^{N} \delta_{i, j} \tau_{i, j}$. For convenience, the notation $|\boldsymbol{\delta}|_{\mathbb{A}(d)}$ represents the tensor norm in the $\mathbb{A}$ metric: $|\boldsymbol{\delta}|_{\mathbb{A}(d)}^{2}=(\mathbb{A}(d) \boldsymbol{\delta}): \boldsymbol{\delta}$. The elasticity operator $\mathbb{A}(d)$ is assumed to be symmetric definite positive and thus it is invertible: its inverse is called the compliance operator. The term $\mathscr{I}_{\operatorname{ker}(\operatorname{tr})}(\dot{\gamma})$ in $(2 \mathrm{~b})$ imposes the incompressibility of the material: $\operatorname{ker}(\operatorname{tr})$ denotes the set of traceless tensors. For any convex set $C$, the indicator function $\mathscr{I}_{C}$ is defined by:

$$
\mathscr{I}_{C}(\xi)= \begin{cases}0 & \text { when } \xi \in C \\ +\infty & \text { otherwise }\end{cases}
$$

The indicator function of a convex set is also convex but not differentiable.

In (2b), the notation with square brackets [.], as in $\phi\left(\left[\gamma, \gamma_{p}, d\right] ; \dot{\gamma}, \dot{\gamma}_{p}, \dot{d}\right)$ indicates a dependence of the dissipation potential upon the state variables $\gamma, \gamma_{p}$ and $d$ as parameters: it is distinct from $\dot{\gamma}, \dot{\gamma}_{p}$ and $\dot{d}$ which are the thermodynamic rate variables of the dissipation potential.

Finally, the potentials $\phi_{p}$ and $\phi_{d}$ describe respectively the viscoplasticity and the damage and $\eta_{s}$ is the bulk viscosity. We assume that both $\phi_{p}$ and $\phi_{d}$ are positive, convex and vanish in zero. Some practical choices for them will be discussed in details in the next paragraph. We are now able to prove the following major result.

Theorem 1 (second principle of thermodynamics and Onsager symmetries). Assume that the dissipation potential $\phi$ is convex, positive and vanishes when the rate variables are zero. Then, the rheological model defined by (2a)-(2b) satisfies both the second principle of thermodynamics and a generalized Onsager symmetry principle.

Proof: The second principle of thermodynamics is directly obtained from [51, p. 223]. From [23, p. 40], the generalized Onsager symmetry principle is obtained as a direct consequence of the present formalism based on the dissipation potential.

\subsection{Constitutive equations}

The constitutive equations are obtained by derivation of the specific free energy and the potential of dissipation by (see [51, p. 223]):

$$
\begin{aligned}
\sigma=\rho \frac{\partial \psi}{\partial \boldsymbol{\gamma}}\left(\boldsymbol{\gamma}, \boldsymbol{\gamma}_{p}, d\right)+\frac{\partial \phi}{\partial \dot{\boldsymbol{\gamma}}}\left(\dot{\boldsymbol{\gamma}}, \dot{\boldsymbol{\gamma}}_{p}, \dot{d}\right) & =\mathbb{A}(d) \boldsymbol{\gamma}_{e}+\partial \mathscr{I}_{\operatorname{ker}(\operatorname{tr})}(\dot{\boldsymbol{\gamma}})+2 \eta_{s} \dot{\boldsymbol{\gamma}} \\
0 \in \rho \frac{\partial \psi}{\partial \gamma_{p}}\left(\boldsymbol{\gamma}, \gamma_{p}, d\right)+\frac{\partial \phi}{\partial \dot{\gamma}_{p}}\left(\dot{\boldsymbol{\gamma}}, \dot{\gamma}_{p}, \dot{d}\right) & =-\mathbb{A}(d) \boldsymbol{\gamma}_{e}+\partial \phi_{p}\left([d], \dot{\boldsymbol{\gamma}}-\dot{\gamma}_{e}\right) \\
0 \in \rho \frac{\partial \psi}{\partial d}\left(\boldsymbol{\gamma}, \boldsymbol{\gamma}_{p}, d\right)+\frac{\partial \phi}{\partial \dot{d}}\left(\dot{\boldsymbol{\gamma}}, \dot{\gamma}_{p}, \dot{d}\right) & =\left(\mathbb{A}^{\prime}(d) \boldsymbol{\gamma}_{e}\right): \boldsymbol{\gamma}_{e}+\partial \phi_{d}\left(\left[\boldsymbol{\gamma}_{e}, d\right] ; \dot{d}\right)
\end{aligned}
$$


where $\boldsymbol{\sigma}$ denotes the total Cauchy stress tensor and we have used $\gamma_{e}=\gamma-\gamma_{p}$ from (1). Also, $\mathbb{A}^{\prime}(d)$ denotes the derivative of the elasticity operator versus $d$. Here $\partial \phi_{d}\left(\left[\gamma_{e}, d\right] ; \dot{d}\right)$ is the subdifferential of $\phi_{d}$ with respect to the variable $\dot{d}$. From proposition 17 in appendix:

$$
\partial \mathscr{I}_{\operatorname{ker}(\mathrm{tr})}(\dot{\gamma})=\{-p \boldsymbol{I} ; p \in \mathbb{R}\}
$$

i.e. the set of spherical tensors. After rearrangements, the three constitutive equations become:

$$
\begin{aligned}
& \boldsymbol{\sigma}=-p \boldsymbol{I}+2 \eta_{s} \dot{\boldsymbol{\gamma}}+\mathbb{A}(d) \boldsymbol{\gamma}_{e} \\
& \partial \phi_{p}\left([d], \dot{\gamma}-\dot{\gamma}_{e}\right) \ni \mathbb{A}(d) \boldsymbol{\gamma}_{e} \\
& \partial \phi_{d}\left(\left[\boldsymbol{\gamma}_{e}, d\right] ; \dot{d}\right) \ni\left(-\mathbb{A}^{\prime}(d) \boldsymbol{\gamma}_{e}\right): \boldsymbol{\gamma}_{e}
\end{aligned}
$$

The first equation (3a) expresses the total Cauchy stress tensor $\boldsymbol{\sigma}$ as the sum of a pressure term, a viscous one and an elastic extra stress contribution. This third contribution involves both the elastic deformation $\gamma_{e}$ and the damage $d$, which are provided by the two last constitutive relations Let us review them.

The second constitutive relation (3b) appears as an implicit differential relation for $\gamma_{e}$, for any given $\dot{\gamma}$. It can be rearranged in a more convenient way, suitable for numerical computations. Let us introduce the elastic stress $\boldsymbol{\sigma}_{e}$ as the dual variable associated to the state variable $\dot{\gamma}_{p}$, i.e. $\boldsymbol{\sigma}_{e}=-\rho \partial \phi_{p} / \partial \dot{\gamma}_{p}=\mathbb{A}(d) \gamma_{e}$. Then (3b) writes equivalently $\boldsymbol{\sigma}_{e} \in \partial \phi_{p}\left([d], \dot{\gamma}_{p}\right)$ or also equivalently $\dot{\gamma}_{p} \in \partial \phi_{p}^{*}\left([d], \boldsymbol{\sigma}_{e}\right)$ thanks to the Fenchel-Young theorem 4 and where $\phi_{p}^{*}$ denotes the convex conjugate of $\phi_{p}$, defined for any $\tau \in \mathbb{R}_{s}^{N \times N}$ by the Legendre transformation (see definition 18). We assume that $\phi_{p}^{*}$ is continuously differentiable, which is the case for our practical choices of this potential. Thus (3b) becomes $\dot{\gamma}_{p}=\nabla \phi_{p}^{*}\left([d] ; \boldsymbol{\sigma}_{e}\right)$, or equivalently, using (1):

$$
\dot{\gamma}_{e}+\nabla \phi_{p}^{*}\left([d] ; \mathbb{A}(d) \gamma_{e}\right)=\dot{\gamma}
$$

Note that, for some given $d$ and $\dot{\gamma}$, relation (3d) appears to be an explicit nonlinear time-differential equation in terms of $\gamma_{e}$, which is suitable to numerical computations. Thanks to the continuity of $\nabla \phi_{p}^{*}$, the existence of a solution for (3d), for some given $d$ and $\dot{\gamma}$, is guaranteed by the CauchyPeano-Arzelà theorem.

The third relation (3c) also appears as an implicit evolution equation for $d$. The strain energy release rate $Y$ is defined as the dual variable associated to the state variable $d$, i.e. $Y=-\rho \partial \psi / \partial d=\left(-\mathbb{A}^{\prime}(d) \gamma_{e}\right): \gamma_{e}$. This concept, set forth by Erdogan and Sih [16], is a natural and obvious generalization of Griffith's [21] original energy release rate that explains the extension of cracks in a material (see e.g. [37, p. 41]). Then, (3c) writes $Y \in \partial \phi_{d}\left(\left[\gamma_{e}, d\right] ; \dot{d}\right)$ or equivalently $\dot{d} \in \partial \phi_{d}^{*}\left(\left[\gamma_{e}, d\right] ; Y\right)$ with the convex conjugate $\phi_{d}^{*}$. Assuming that $\phi_{p}^{*}$ is continuously differentiable, which is the case for our practical choices of this potential, then (3c) expresses as an explicit timedifferential equation in terms of the damage $d$ :

$$
\dot{d}=\nabla \phi_{d}^{*}\left(\left[\gamma_{e}, d\right] ;\left\{-\mathbb{A}^{\prime}(d) \gamma_{e}\right\}: \gamma_{e}\right)
$$

Remark 1 (undamaged Maxwell model).

Let us choose the damage potential $\phi_{d}=0$. Then, from (3e), assuming $d=0$ at $t=0$, the material remains undamaged at any time. Moreover, choosing the elasticity operator $\mathbb{A} \gamma_{e}=G \gamma_{e}$ and the viscoplastic potential $\phi_{p}(\boldsymbol{\delta})=\eta|\boldsymbol{\delta}|^{2}$ for any $\gamma_{\boldsymbol{e}}$ and $\boldsymbol{\delta} \in \mathbb{R}_{s}^{N \times N}$, with $G>0$ and $\eta>0$, then the rheological model (2a)-(2b) coincides with the Maxwell viscoelastic model [41], as extended by Oldroyd [45] in a tensor framework, and with a characteristic relaxation time equal to $\eta / G$.

\subsection{Clausius-Duhem inequality}

An equivalent expression of the second principle of thermodynamics, stated in theorem 1 , is the Clausius-Duhem inequality (see e.g. [51, p. 221]), that writes here, since the process is isothermal, 
as:

$$
w=-\rho \dot{\psi}+\sigma: \dot{\gamma} \geqslant 0
$$

where $w$ denotes the total dissipation. From (1) and (2a), the first term expands as:

$$
-\rho \dot{\psi}=-\rho \frac{\partial \psi}{\partial \boldsymbol{\gamma}}: \dot{\gamma}-\rho \frac{\partial \psi}{\partial \gamma_{p}}: \dot{\gamma}_{p}-\rho \frac{\partial \psi}{\partial d} \dot{d}=-\boldsymbol{\sigma}_{e}: \dot{\gamma}_{e}+Y \dot{d}
$$

where $\boldsymbol{\sigma}_{e}$ is the elastic stress and $Y$ the strain energy release rate. Then, using (3a), the dissipation becomes $w=2 \eta_{s}|\dot{\gamma}|^{2}+\sigma_{e}: \dot{\gamma}_{p}+Y \dot{d}$. Observe that

$$
\begin{aligned}
& w_{p}=2 \eta_{s}|\dot{\gamma}|^{2}+\sigma_{e}: \dot{\gamma}_{p}=2 \eta_{s}\left|\boldsymbol{\sigma}_{e}\right|^{2}|\dot{\gamma}|^{2}+\sigma_{e}: \nabla \phi_{p}^{*}\left([d], \sigma_{e}\right) \geqslant 0 \\
& w_{d}=Y \dot{d}=Y \nabla \phi_{d}^{*}\left(\left[\gamma_{e}, d\right] ; Y\right) \geqslant 0
\end{aligned}
$$

where we have used (3b) and (3c). Note that the positivity of both $w_{p}$ and $w_{d}$ is a direct consequence of the convexity of the two positive potentials $\phi_{p}$ and $\phi_{d}$ vanishing in zero. Then $w=w_{p}+w_{d} \geqslant 0$. The first term $w_{p}$ represents the dissipation due to viscoplastic effects and the second one $w_{d}$, the dissipation due to damage. For the present mathematical model, we finally obtain a stronger proposition than the second principle (theorem 1): each of the two contributions to the dissipation are separately positive.

\subsection{General problem statement}

The three constitutive equations (3a), (3d) and (3e) are coupled here with the conservation of mass and momentum. The deformation rate $\dot{\gamma}$ is identified as the symmetric part of the velocity gradient tensor $D(\boldsymbol{u})=\left(\nabla \boldsymbol{u}+\nabla \boldsymbol{u}^{T}\right) / 2$, where $\boldsymbol{u}$ denotes the velocity of the material and $\nabla \boldsymbol{u}=\left(\partial u_{i} / \partial x_{j}\right)_{1 \leqslant i, j \leqslant N}$ is the gradient of velocity tensor. Conversely, the time derivative $\dot{\gamma}_{e}$ is replaced by the upper-convected tensor derivative $\stackrel{\nabla}{\gamma}_{e}$. Assuming a constant density $\rho$, the mass conservation coincides with the incompressibility constraint. The problem expresses as a system of four equations for four unknowns:

$(P)$ : find the elastic deformation $\boldsymbol{\gamma}_{e}$, the damage $d$, the velocity $\boldsymbol{u}$ and the pressure $p$ satisfying

$$
\left\{\begin{array}{r}
\stackrel{\nabla}{\boldsymbol{\gamma}}_{e}+\nabla \phi_{p}^{*}\left([d] ; \mathbb{A}(d) \boldsymbol{\gamma}_{e}\right)-D(\boldsymbol{u})=0 \\
\frac{\partial d}{\partial t}+(\boldsymbol{u} \cdot \nabla) d=\nabla \phi_{d}^{*}\left(\left[\boldsymbol{\gamma}_{e}, d\right] ;\left(-\mathbb{A}^{\prime}(d) \boldsymbol{\gamma}_{e}\right): \boldsymbol{\gamma}_{e}\right) \\
\rho\left(\frac{\partial \boldsymbol{u}}{\partial t}+(\boldsymbol{u} \cdot \nabla) \boldsymbol{u}\right)-\operatorname{div}\left(-p \boldsymbol{I}+2 \eta_{s} D(\boldsymbol{u})+\mathbb{A}(d) \boldsymbol{\gamma}_{e}\right)=\boldsymbol{f} \\
\operatorname{div} \boldsymbol{u}=0
\end{array}\right.
$$

where $f$ is some given external force applied. This set of equations is closed by suitable initial and boundary conditions. The material functions $\mathbb{A}, \phi_{p}$ and $\phi_{d}$ are still quite general. In the rest of the paper, possible choices for these material functions are discussed.

Remark 2 (objective tensor derivatives). Note that, in (5a), a Gordon-Schowalter [20] tensor derivative could be used instead of the upperconvected one (see also [51, p. 150])). The Gordon-Schowalter derivative introduces an additional material parameter that interprets as a control of the slip of the micro-structure with respect to the macro-scale frame. Recall that both the upper-convected and the Jaumann tensor derivatives are obtained as a special case of it. See e.g. [22] for some discussion about material derivatives in the context of plasticity of solids in large deformations and $[48,7]$ for some numerical experiences of the Gordon-Schowalter derivative in the context of viscoelastic fluids. 
Remark 3 (plastic strain rate).

Observe that (1) leads to $\dot{\gamma}_{e}+\dot{\gamma}_{p}=\dot{\gamma}$, i.e. a decomposition of the deformation rate. Since the deformation rate $\dot{\gamma}$ is identified as the symmetric part of the velocity gradient tensor $D(\boldsymbol{u})$ while the elastic deformation rate $\dot{\gamma}_{e}$ is replaced by $\stackrel{\nabla}{\gamma}_{e}$, then, from the constitutive equation (5a), the plastic deformation rate $\dot{\gamma}_{p}$ coincides with $\nabla \phi_{p}^{*}\left([d] ; \mathbb{A}(d) \boldsymbol{\gamma}_{e}\right)$. Remark that this plastic deformation rate mainly depends upon the elastic stress $\sigma_{e}=\mathbb{A}(d) \gamma_{e}$. See e.g. [28] for a discussion on the plastic deformation rate for elastoviscoplastic materials and its possible dependence upon other fields.

\subsection{Example: the BMP thixotropic model}

This model, first introduced in [5], combines the Maxwell viscoelatic model with the kinetic equation proposed by [17], for destruction and construction of structure. It presents three independent thermodynamic variables: the total deformation $\gamma$, the plastic deformation $\gamma_{p}$, and the fluidity $\varphi$, that has the dimension of the inverse of a viscosity. The fluidity acts here similarly to the previous damage variable $d$ : indeed, it describes the microscopic state of the material. The free energy and the dissipation potential are given by

$$
\begin{aligned}
\psi\left([\dot{\gamma}] ; \gamma, \gamma_{p}, \varphi\right) & =\frac{G}{\rho}\left|\gamma-\gamma_{p}\right|^{2} \\
& +\frac{\alpha}{\rho}\left(\frac{\varphi^{2}}{2}-\varphi_{0} \varphi\right)+\frac{\beta}{\rho}\left(\frac{\varphi^{2}}{2}-\varphi_{\infty} \varphi\right)\left(\gamma-\gamma_{p}\right): \dot{\gamma} \\
\phi\left([\varphi] ; \dot{\gamma}, \dot{\gamma}_{p}, \dot{\varphi}\right) & =\mathscr{I}_{\operatorname{ker}(\operatorname{tr})}(\dot{\gamma})+\varphi^{-1}\left|\dot{\gamma}_{p}\right|^{2}+\frac{c_{f}}{2} \dot{\varphi}^{2}
\end{aligned}
$$

where $G, \varphi_{0}, \varphi_{\infty}, \alpha, \beta$ and $c_{f}$ are given positive constants with $0 \leqslant \varphi_{0}<\varphi_{\infty}$. Recall the notation with square brackets: it indicates the dependence of the free energy and the dissipation potential upon parameters. The $\mathscr{I}_{\operatorname{ker}(\operatorname{tr})}$ term imposes a traceless rate of deformation $\dot{\gamma}$, i.e. an incompressible fluid (see proposition 17 in appendix).

Observe that theorem 1 applies and then both the second principle of thermodynamics and the Onsager symmetry are satisfied. Moreover, results of paragraph 1.3 apply and, from (4a)-(4b), the dissipation writes $w=4 G^{2} \varphi\left|\gamma_{e}\right|^{2}+c_{f} \dot{\varphi}^{2} \geqslant 0$. Note that $Y=\partial \phi / \partial \dot{\varphi}=c_{f} \dot{\varphi}$ is the dual variable associated to the fluidity $\dot{\varphi}$. The first term in the expression of $w$ represents the dissipation due to viscoelastic effects and the second one, those due to changes in the microstructure. Note that both are positive. To our best knowledge, these results was not yet stated for the BMP model.

Next, let us expand the constitutive equations. Using $\gamma_{e}=\gamma-\gamma_{p}$, the constitutive equations write (see [51, p. 223]):

$$
\begin{aligned}
\boldsymbol{\sigma} & =-p \boldsymbol{I}+2 G \boldsymbol{\gamma}_{e} \\
0 & =-2 G \boldsymbol{\gamma}_{e}+2 \varphi^{-1}\left(\dot{\gamma}-\dot{\gamma}_{e}\right) \\
0 & =\alpha\left(\varphi-\varphi_{0}\right)+\beta\left(\varphi-\varphi_{\infty}\right)\left(\gamma-\gamma_{p}\right): \dot{\gamma}+c_{f} \dot{\varphi}
\end{aligned}
$$

where $p$ is the pressure that acts as a Lagrange multiplier for imposing the fluid incompressibility (see proposition 17 in appendix). These equations write equivalently

$$
\begin{aligned}
& \boldsymbol{\sigma}=-p \boldsymbol{I}+\boldsymbol{\tau} \\
& \quad \dot{\boldsymbol{\tau}}+\varphi \boldsymbol{\tau}=2 \dot{\boldsymbol{\gamma}} \\
& \dot{G}+\frac{\varphi-\varphi_{0}}{\lambda}+k\left(\varphi-\varphi_{\infty}\right) \boldsymbol{\tau}: \dot{\gamma}=0
\end{aligned}
$$

where we have introduced the notations $\boldsymbol{\tau}=2 G \gamma_{e}, \lambda=c_{f} / \alpha$ and $k=\beta /\left(c_{f} G\right)$. Finally, replacing $2 \dot{\boldsymbol{\gamma}}$ by $D(\boldsymbol{u})$ and $\dot{\boldsymbol{\tau}}$ by the upper-convected tensor derivative, we exactly obtain relations (1)-(2) of the BMP model, as formulated in [5]. See also [39] for a different thermodynamic approach of the BMP model and [18] for an investigation of the yield stress limit $\varphi_{0}=0$. 


\section{Practical choices for the material functions}

In this section, the previous theoretical framework is instantiated for building a practical rheological model that combines viscous effects with elasticity, plasticity and damage. The elasticity is presented first, with a discussion on the Poisson ratio. Then, plasticity effects are introduced, with a in-depth exploration of the Drucker-Prager criterion which describes at the macroscopic level the friction between grains at the microscopic one. Damage evolution is then introduced, with a yield criterion similar to the plasticity one. Merging all together, the obtained rheological model is compared with previous existing models and could be considered an extension of several of them.

\subsection{Choosing the elasticity operator $\mathbb{A}$ versus the damage}

Here, we assume the material to be isotropic. Then, from [30], the linear elasticity operator $\mathbb{A}(d)$ expresses, for any $\boldsymbol{\delta} \in \mathbb{R}_{s}^{N \times N}$, as:

$$
\mathbb{A}(d) \boldsymbol{\delta}=2 G(d) \boldsymbol{\delta}+\lambda(d)(\operatorname{tr} \boldsymbol{\delta}) \boldsymbol{I}
$$

where $\lambda(d)$ and $G(d)$ are the Lamé coefficients of the damaged material and $\operatorname{tr}($.$) denotes the trace$ of a matrix. The Lamé coefficients express equivalently in terms of the elastic modulus $E(d)$ and the Poisson ratio $\nu(d)$ (see e.g. [34, p. 107]):

$$
\lambda(d)=\frac{E(d) \nu(d)}{(1+\nu(d))(1-2 \nu(d))} \text { and } G(d)=\frac{E(d)}{2(1+\nu(d))}
$$

Kachanov [33] defined the damage variable $d$ from the elastic modulus $E(d)$, assumed to vary linearly versus $1-d$, i.e.

$$
E(d)=(1-d) E_{0}
$$

where $E_{0}>0$ is the elastic modulus associated to the undamaged material. The simplest choice for the Lamé coefficients would be to choose them proportional to $(1-d)$, with the proportionality constants i.e. $\lambda(d)=(1-d) \lambda_{0}$ and $G(d)=(1-d) G_{0}$ where $\lambda_{0}$ and $G_{0}$ are the Lamé coefficients associated to the the undamaged material. With this choice, observe that $\mathbb{A}(d)=(1-d) \mathbb{A}(0)$ and then $\left(-\mathbb{A}^{\prime}(d) \gamma_{e}\right): \gamma_{e}=2\left|\gamma_{e}\right|_{\mathbb{A}(0)}^{2} \geqslant 0$. This quantity, involved in the right-hand-side of the damage equation (3e), represents the elastic energy of the undamaged material.

This simple choice leads to a constant Poisson ratio $\nu(d)$, which is in disagreement with many experimental observations, as pointed out by $\mathrm{Ju}$ [32]. Indeed, the Poisson ratios is expected to increase under increasing damage [25, 26], as a result of micro-cracking. For this reason, this author suggested extending the damage variable $d$ from a scalar to a tensor quantity (see also [24]). See [53] for a discussion about the variation of the Poisson ratio versus damage. We consider here the Poisson ratio to depends linearly upon $d$ i.e

$$
\nu(d)=\nu_{0}+\left(\nu_{1}-\nu_{0}\right) d
$$

with $-1<\nu_{0} \leqslant \nu_{1}<1 / 2$. Observe also that when $\nu_{1}=\nu_{0}$, this choice coincides with the previous one, when are simply $\lambda(d)$ and $G(d)$ proportional to $1-d$. Otherwise, when $\nu_{1}>\nu_{0}$, then the Poisson ratio is an increasing function of the damage, which is the expected behavior.

\subsection{Choosing the viscoplastic potential $\phi_{p}$}

The Drucker-Prager [14] plasticity criterion considers the translated cone defined by

$$
T_{\mu, \sigma_{y}}=\left\{\boldsymbol{\tau} \in \mathbb{R}_{s}^{N \times N} ;|\operatorname{dev} \boldsymbol{\tau}|-\sigma_{y} \leqslant \frac{\mu}{\sqrt{N}} \operatorname{tr} \boldsymbol{\tau}\right\}
$$



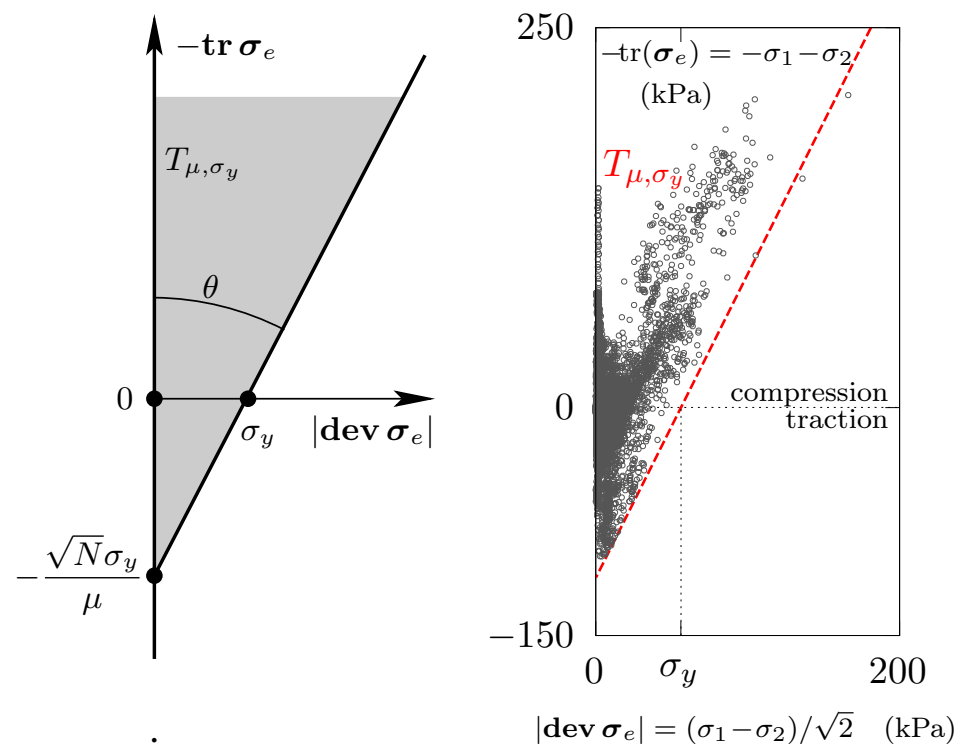

Figure 2: (left) The viscoplastic Drucker-Prager translated cone $T_{\mu, \sigma_{y}}$. (right) The Drucker-Prager translated cone $T_{\mu, \sigma_{y}}$ together with experimental data represented by circles (from [56], Fig. 13, Baltimore in-situ sea ice station). Adjusted parameters are $\mu=1 / \sqrt{2}$ and $\sigma_{y}=56 \mathrm{kPa}$ for $N=2$.

where $\sigma_{y}$ is the cohesion and $\mu \in[0, \infty[$ is the friction coefficient. The notation $\operatorname{dev} \boldsymbol{\delta}=\boldsymbol{\delta}-(1 / N)(\operatorname{tr} \boldsymbol{\delta}) \boldsymbol{I}$ represents the deviatoric part of any matrix $\boldsymbol{\delta}$. This cone is represented in the stress plane on Fig. 2.left. Its boundary defines a straight line that intercepts the vertical axis at $-\sqrt{N} \sigma_{y} / \mu$ and has slope $\sqrt{N} / \mu$. The angle $\theta$ that this line makes with the vertical axis is given by $\theta=\tan ^{-1}(\mu / \sqrt{N})$ and is known as the angle of internal friction [29, p. 90]. Fig. 2.right represents in-situs sea ice observation [56] together with the Drucker-Prager cone. When the elastic stress goes outside of this cone, the material develops irreversible deformations, and then the elastic stress relaxes and goes back inside of the cone. Observe for $N=2$ that $\mu \approx 1 / \sqrt{2}$ and $\theta=\tan ^{-1}(1 / 2) \approx 27^{\circ}$.

The viscoplastic part $\phi_{p}$ of the total dissipation potential $\phi$ is then defined for all $\delta \in \mathbb{R}_{s}^{N \times N}$ by

$$
\phi_{p}([d] ; \boldsymbol{\delta})=\eta(d)|\boldsymbol{\delta}|^{2}+\left(\mathscr{I}_{-T_{\mu, \sigma_{y}}}\right)^{*}(\boldsymbol{\delta})
$$

The first term represents viscous effects. The second term is the expression in terms of convex analysis of the classical Drucker-Prager plasticity criterion [14] with cohesion [1]. This formalism was first introduced by Saxcé and coworkers [27, p. 1116] for applications to solid elastoplastic materials. Here, $\mathscr{I}_{-T_{\mu, \sigma}}$ denotes the indicator to the translated Drucker-Prager cone (see proposition 41). Note that the evolution equation (3d) for the elastic deformation $\gamma_{e}$ involves $\nabla \phi_{p}^{*}$ which is provided by the following result.

Theorem 2 (viscoplastic Drucker-Prager).

Let $\phi_{p}$ be the viscoplastic Drucker-Prager potential defined by (6e). Its convex conjugate $\phi_{p}^{*}$ is continuous and differentiable, and its gradient $\nabla \phi_{p}^{*}$ is also continuous and expresses, for all $\boldsymbol{\tau} \in \mathbb{R}_{s}^{N \times N}$, as

$$
\nabla \phi_{p}^{*}(\boldsymbol{\tau})=\frac{\kappa_{\mu, \sigma_{y}}(\boldsymbol{\tau})}{2 \eta\left(1+\mu^{2}\right)}\left(\boldsymbol{\tau}-\frac{\xi_{\mu, \sigma_{y}}(\boldsymbol{\tau})}{\sqrt{N} \mu} \boldsymbol{I}\right)
$$




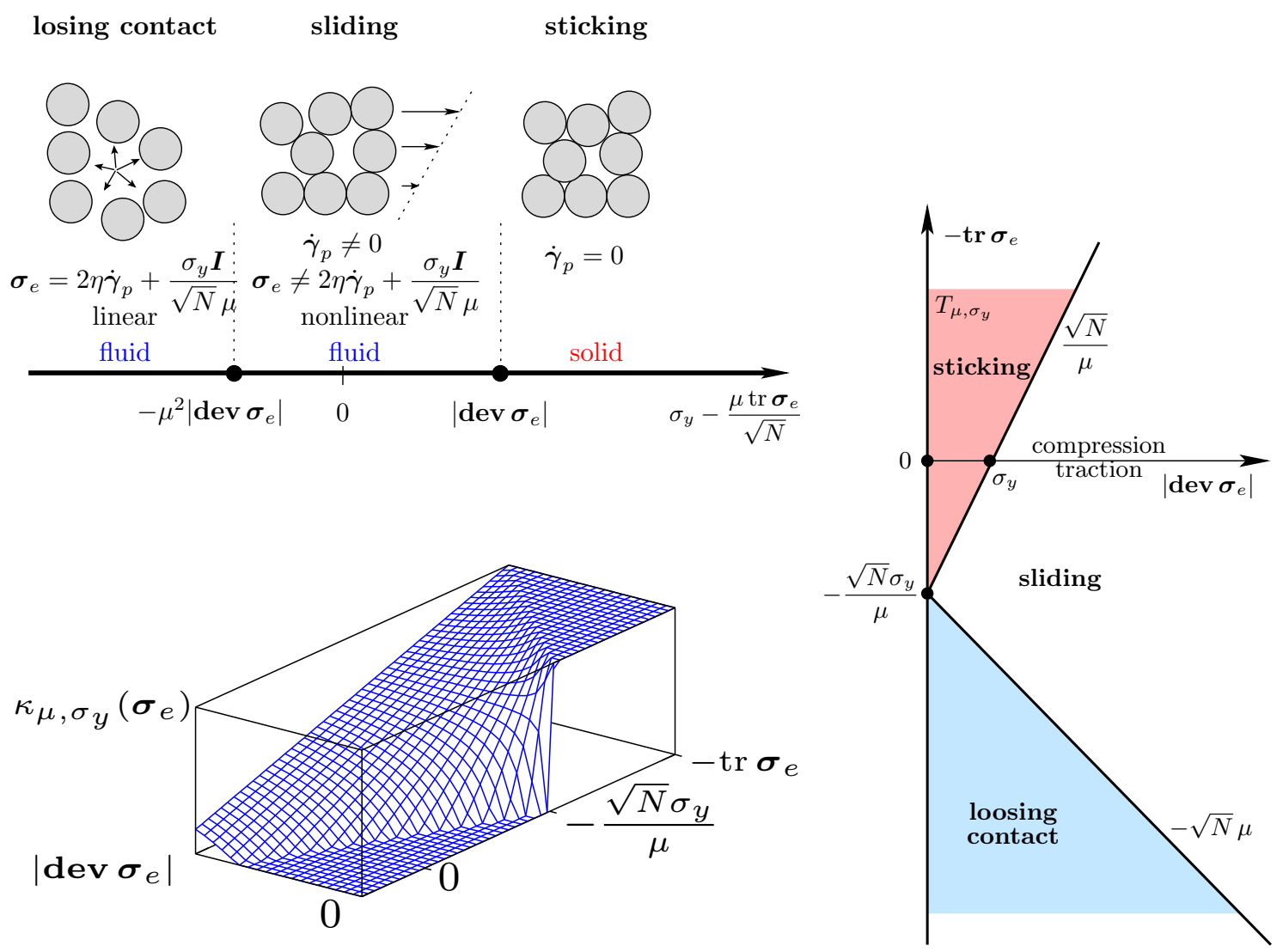

Figure 3: Representation of the viscoplastic constitutive equation $\dot{\gamma}_{p}=\nabla \phi_{p}^{*}\left(\boldsymbol{\sigma}_{e}\right)$. (top-left) The three flow regimes, depending upon the pressure $-\operatorname{tr} \boldsymbol{\sigma}_{e} / N$. (right) Representation in the stress plane. (bottom-left) Elevation of the $\kappa_{\mu, \sigma_{y}}$ function in the stress plane.

where

$$
\begin{aligned}
& \kappa_{\mu, \sigma_{y}}(\boldsymbol{\tau})= \begin{cases}1+\mu^{2} & \text { when }-\mu^{2}|\operatorname{dev} \boldsymbol{\tau}| \geqslant \sigma_{y}-\frac{\mu}{\sqrt{N}} \operatorname{tr} \boldsymbol{\tau} \\
1-\frac{\sigma_{y}-\frac{\mu}{\sqrt{N}} \operatorname{tr} \boldsymbol{\tau}}{|\operatorname{dev} \boldsymbol{\tau}|} & \text { when }-\mu^{2}|\operatorname{dev} \boldsymbol{\tau}|<\sigma_{y}-\frac{\mu}{\sqrt{N}} \operatorname{tr} \boldsymbol{\tau}<|\operatorname{dev} \boldsymbol{\tau}|(6 \mathrm{~g}) \\
0 & \text { otherwise }\end{cases} \\
& \xi_{\mu, \sigma_{y}}(\boldsymbol{\tau})=\min \left(\sigma_{y}, \frac{\mu \operatorname{tr} \boldsymbol{\tau}}{\left.\sqrt{N}-\mu^{2}|\operatorname{dev} \boldsymbol{\tau}|\right)}\right.
\end{aligned}
$$

While the expression (6f) of $\nabla \phi_{p}^{*}$ is quite concise, the proof of theorem 2 requires some technical developments and is postponed in Appendix A. The elevation view on Fig. 3.bottom-left shows that $\kappa_{\mu, \sigma_{y}}$ is continuous except at the junction between the three cones and is differentiable except along the cone boundaries. At this junction, the second factor in (6f) vanishes and finally, $\nabla \phi_{p}^{*}$ is continuous everywhere. Recall that the potential $\phi_{p}$ involves three parameters: $\eta, \sigma_{y}$ and $\mu$. Note that, for simplicity, the dependence of the coefficients $\eta$ and $\sigma_{y}$ upon the damage $d$ has been omitted in the statement of this result. When $\mu=0$, the present potential describes the 
viscoplastic Bingham constitutive equation (see e.g. [49], eqn (2)):

$$
\nabla \phi_{p}^{*}(\boldsymbol{\tau})=\left\{\begin{array}{cl}
\left(1-\frac{\sigma_{y}}{|\operatorname{dev} \boldsymbol{\tau}|}\right) \frac{\boldsymbol{\tau}}{2 \eta} & \text { when }|\operatorname{dev} \boldsymbol{\tau}|>\sigma_{y} \\
0 & \text { otherwise }
\end{array}\right.
$$

and then, the cohesion $\sigma_{y}$ coincides with the usual yield stress. Finally, in the case $\mu=0$, the evolution equation (3d) for $\dot{\gamma}_{e}$ coincides with the elastoviscoplastic model, as introduced in [49].

When $\mu>0$, from theorem 2, the constitutive equation $\dot{\gamma}_{p}=\nabla \phi_{p}^{*}\left(\boldsymbol{\sigma}_{e}\right)$ develops three flow regimes, as represented on Fig. 3.top-left. As expected, in compression and when the elastic stress $\boldsymbol{\sigma}_{e}$ belongs to the Drucker-Prager translated cone (in red on Fig. 3.right), the material behaves as a solid i.e. the irreversible deformation rate $\dot{\gamma}_{p}=0$ and this is the sticking regime. In that case, the material behaves as an incompressible Kelvin-Voigt viscoelastic solid and, from (1) and (3a) we get $\boldsymbol{\sigma}=-p \boldsymbol{I}+2 \eta_{s} \dot{\boldsymbol{\gamma}}+\mathbb{A} \boldsymbol{\gamma}$. When the elastic stress $\boldsymbol{\sigma}_{e}$ lives outside of the Drucker-Prager translated cone, material behaves as a fluid, i.e. the irreversible deformation rate $\dot{\gamma}_{p} \neq 0$. In that case, the situation is here more subtle than for Bingham viscoplasticity as there are now still two distinct flow regimes. When the elastic stress $\boldsymbol{\sigma}_{e}$ belongs to the blue cone on Fig. 3.right, i.e. when the traction is sufficient, this is the losing contact regime. We then have $\boldsymbol{\sigma}_{e}=2 \eta \dot{\boldsymbol{\gamma}}_{p}+\left(\sigma_{y} /(\sqrt{N} \mu)\right) \boldsymbol{I}=\mathbb{A} \boldsymbol{\gamma}_{e}$ i.e. the material is a viscoelastic fluid of Oldroyd kind. Indeed, from (1) and (3a) we obtain $\boldsymbol{\sigma}=-p \boldsymbol{I}+2 \eta_{s} \dot{\boldsymbol{\gamma}}+\mathbb{A} \boldsymbol{\gamma}_{e}$ and, from (5a):

$$
\stackrel{\nabla}{\gamma}_{e}+\frac{1}{2 \eta}\left(\mathbb{A} \boldsymbol{\gamma}_{e}-\frac{\sigma_{y}}{\sqrt{N} \mu} \boldsymbol{I}\right)=\dot{\gamma}
$$

Observe that, in that case, the elastic stress $\boldsymbol{\sigma}_{e}=\mathbb{A} \boldsymbol{\gamma}_{e}$ relaxes to $\sigma_{y} /(\sqrt{N} \mu) \boldsymbol{I}$ which locates on the vertical axis, exactly at the junction between the three cones on Fig. 3.right. Finally, when the elastic stress $\boldsymbol{\sigma}_{e}$ lives in the white cone between the sticking and the losing contact regions, the material behaves as a complex nonlinear viscoelastic fluid.

This material could be interpreted in terms of a granular microstructure suspended in a bulk fluid (see Fig. 3.top-left). In the sticking regime, the grains are sticking together and the collective behavior is a solid one. For the sliding regime, the grains are moving while maintaining frictional contacts. For the losing contact regime, the microstructure corresponds to a granular gas suspended in a bulk fluid.

Note that the viscoplastic potential $\phi_{p}$ introduced here shares many similarities with the viscoplastic model developed by Daviet and Bertails [11] (see also [10]). These authors introduced a truncated cone (see [11, p. 18], Fig. 2) while the cone $T_{\mu, \sigma_{y}}$ is here not truncated, similar to those of Saxcé and coworkers [27, p. 1116]. For instance, when $\mu=0$, these authors obtained a variant of the Bingham model with a dilatancy constraint $\operatorname{div} \boldsymbol{u} \geqslant 0$.

Finally, let us turn to damage effects. While $\mu$ is considered here as constant, the viscosity $\eta$ and the cohesion $\sigma_{y}$ are assumed to vary upon the damage as

$$
\begin{aligned}
\eta(d) & =(1-d) \eta_{0} \\
\sigma_{y}(d) & =(1-d) \sigma_{y 0}
\end{aligned}
$$

where $\eta_{0}>0$ is the viscosity of the undamaged material and $\sigma_{y 0} \geqslant 0$ its cohesion. The next paragraph develops the evolution equation for the damage.

\subsection{Choosing the brittle-damage potential $\phi_{d}$}

A simple damage function - The simplest choice for the damage potential would be $\phi_{d}([d] ; \dot{d})=\eta_{d} \dot{d}^{2} /(1-d)$ where $\eta_{d} \geqslant 0$ has the dimension of a viscosity. Recall that $d$ and $\dot{d}$ 
are considered as independent variable: here, the rate $\dot{d}$ acts as a variable while $d$ is a parameter of the potential. Then $\phi_{d}^{*}([d] ; Y)=(1-d) Y^{2} /\left(4 \eta_{d}\right)$ is such that the Fenchel-Young relation $Y=\nabla \phi_{d}(\dot{d}) \Longleftrightarrow \dot{d}=\nabla \phi_{d}^{*}(Y)$ is satisfied. Replacing in (3c), we would obtain:

$$
\dot{d}=\frac{(1-d)}{2 \eta_{d}}\left(-\mathbb{A}^{\prime}(d) \gamma_{e}\right): \gamma_{e}
$$

Note that the ratio $\eta_{d} / E_{0}$ represents a characteristic time associated to the damage while the $1-d$ prefactor assures that $\nabla \phi_{d}^{*}$ smoothly vanishes at the limit $d=1$. Others expressions of $d$ that vanishes at $d=1$ could be considered similarly. See e.g. [40, p. 211] for alternative power-law index or [37, chap. 3].

Brittle damage based on a yield criterion - Experimental observations showed that the previous damage criterion is unrealistic: damage develops only when the stress goes outside of a given cone. See again Fig. 2.right that represents in-situs sea ice observation [56]. The proposed criterion bases again on the translated Drucker-Prager cone. We now consider that the brittledamage potential $\phi_{d}$ is expressed by defining its convex conjugate:

$$
\phi_{d}^{*}\left(\left[\gamma_{e}, d\right] ; Y\right)=\frac{(1-d) \kappa_{\mu, \sigma_{c}}\left(\mathbb{A}(d) \gamma_{e}\right)}{4 \eta_{d}\left(1+\mu^{2}\right)} Y^{2}
$$

where $\kappa_{\mu, \sigma_{c}}$ is expressed by $(6 \mathrm{~g})$. Here, $\sigma_{c}$ is the yield stress in pure shear, or material cohesion, $\mu$ is the friction coefficient, as for the Drucker-Prager cone. and $\eta_{d}>0$ is a constant that has the dimension of a viscosity. Observe that $\phi_{d}^{*}$ is differentiable and

$$
\nabla \phi_{d}^{*}\left(\left[\gamma_{e}, d\right] ; Y\right)=\frac{(1-d) \kappa_{\mu, \sigma_{c}}\left(\mathbb{A}(d) \boldsymbol{\gamma}_{e}\right)}{2 \eta_{d}\left(1+\mu^{2}\right)} Y
$$

The evolution equation (3e) becomes:

$$
\dot{d}=\frac{(1-d) \kappa_{\mu, \sigma_{c}}\left(\mathbb{A}(d) \boldsymbol{\gamma}_{e}\right)}{2 \eta_{d}\left(1+\mu^{2}\right)}\left(-\mathbb{A}^{\prime}(d) \boldsymbol{\gamma}_{e}\right): \boldsymbol{\gamma}_{e}
$$

where $Y$ has been replaced by its expression. Note that the ratio $\eta_{d} / E_{0}$ still represents a characteristic time associated to the damage. This damage criterion is represented on Fig. 2.right together with in-situs sea ice observation [56]. When the elastic tensor $\boldsymbol{\sigma}_{e}=\mathbb{A}(d) \boldsymbol{\gamma}_{e}$ belongs to the translated Drucker-Prager cone $-T_{\mu, \sigma_{c}}$, then $\kappa_{\mu, \sigma_{c}}\left(\boldsymbol{\sigma}_{e}\right)=0$ and, from (61), the damage do not develop. Otherwise, $\kappa_{\mu, \sigma_{c}}\left(\boldsymbol{\sigma}_{e}\right)>0$ and some damage will occur. For the damage to nicely interact with plasticity, we also assume $\sigma_{c} \geqslant \sigma_{y 0}$. Note that, for bidimensional problems, the Drucker-Prager criterion coincides with the Mohr-Coulomb one, defined in terms of the two real eigenvalues $\sigma_{1}, \sigma_{2}$ of the elastic stress (see e.g. [1]). Indeed $\left|\operatorname{dev} \boldsymbol{\sigma}_{e}\right|=\sqrt{2}\left(\sigma_{1}-\sigma_{2}\right)$ and $\operatorname{tr} \boldsymbol{\sigma}_{e}=\sigma_{1}+\sigma_{2}$. This criterion is widely used in models representing the progressive failure of brittle materials (see e.g. [56]). It recently has been used in the sea-ice coupled viscoelastic-damage model $[8,9]$.

\subsection{The new brittle-elastoviscoplastic model}

Let us group the previous choices (6b) for the elasticity operator and (6e) and (6l) for the viscoplastic and damage the dissipation potentials, respectively. The rheological model is represented on Fig. 1.right. It shares many similarities with a previous elastoviscoplastic (EVP) model [49]: it consists in a dash-pot and a dry-friction elements connected in parallel, together with a spring connected in series. The main differences are (i) the dependence of the rheological parameters upon the damage variable $d$, (ii) the introduction of a Coulomb friction coefficient $\mu$ and (iii) the Poisson ratio $\nu(d)$ for an elastic compressibility. This model shares some similarities with several existing ones, as shown on Fig. 4. When the damage effects are not considered, the present model reduces to an elastoviscoplastic model that extends with a Coulomb friction the authors' previous one [49], and represented on the center axis of Fig. 4. When cohesion and friction are not considered $\left(\sigma_{y}=\mu=0\right)$, the present model reduces to a brittle-viscoelastic one, similar to the MEB 


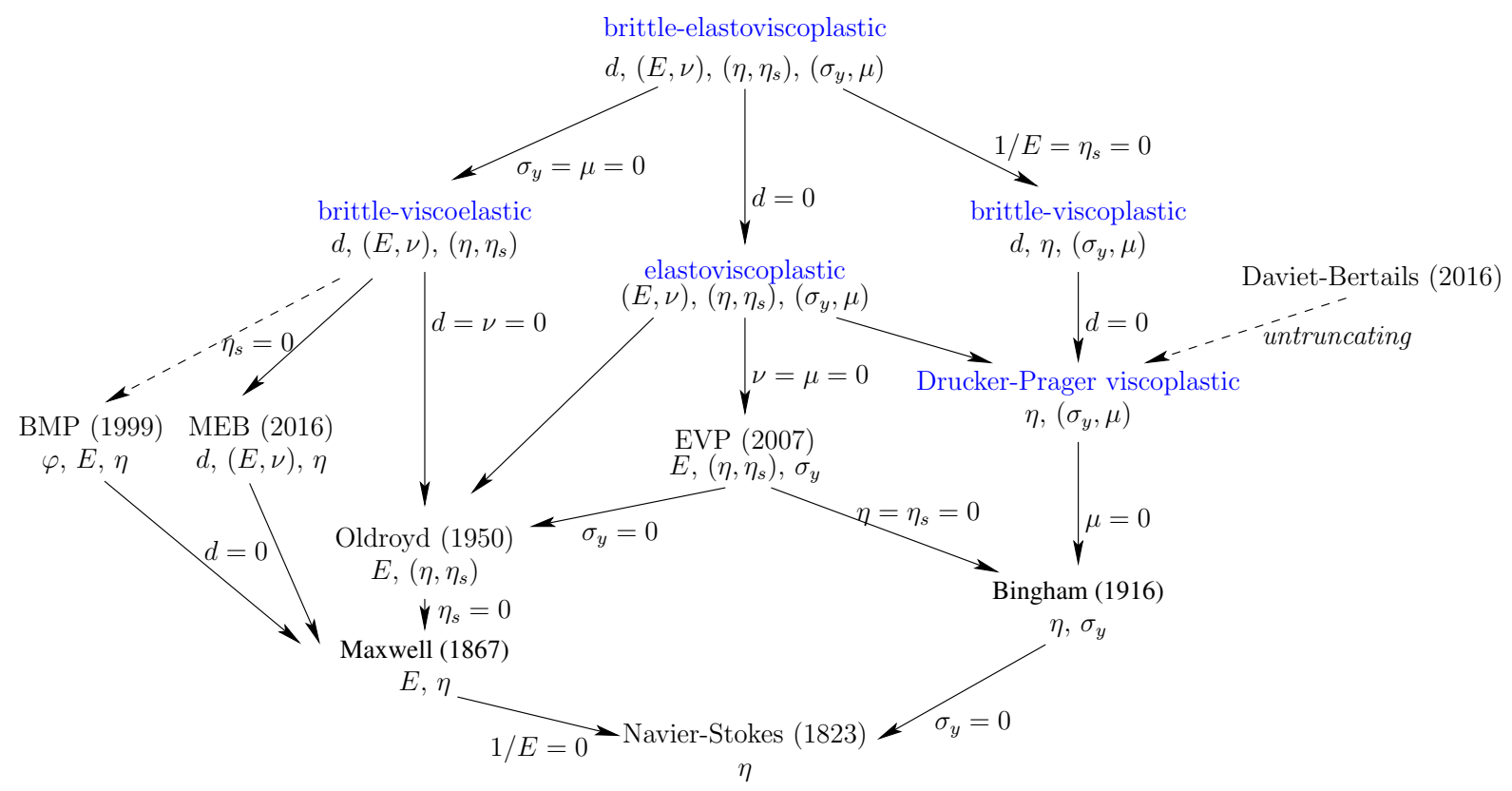

Figure 4: A hierarchy of rheological models, where new models are in blue.

one [8], and represented on the left side of Fig. 4. Note that the mathematical structure then shares some similarities with the BMP model, the damage variable being replaced by the fluidity for the description of thixotropic effects. Both MEB and BMP reduce to the Maxwell viscoelastic model by neglecting damage or thixotropy, respectively. Conversely, on the right side of Fig. 4, when elastic effects are not considered $(1 / E=0)$, the present model reduces to a brittle-viscoplastic material (BVP). This BVP model bases on a new Drucker-Prager viscoplastic (DP-VP) model by including damage effects. This new DP-VP model itself extends the usual Bingham viscoplastic model and shares many similarities with the Daviet and Bertails [11] viscoplastic model based on a truncated translated Drucker-Prager cone (see [11, p. 18], Fig. 2). When neglecting Coulomb friction $(\mu=0)$, the present model then reduces to the usual incompressible Bingham model while the Daviet and Bertails one reduces to a dilatant Bingham (div $\boldsymbol{u} \geqslant 0$ while here $\operatorname{div} \boldsymbol{u}=0$ ).

\section{Results and discussion}

This last section is dedicated to a preliminary exploration of the previously obtained model: the uniaxial compression benchmark is considered. The boundary conditions are described and the material parameters are chosen in order to be representative of applications in geosciences. Next, numerical approximation parameters, such as meshes and time step, are introduced. This section closes with an exploration of the time-dependent solution.

\subsection{Material parameters and flow conditions}

Our aim is to study the interplay between the main nonlinearities introduced by the two dissipation potentials $\phi_{p}$ and $\phi_{d}$, expressed by $(6 \mathrm{e})$ and $(6 \mathrm{k})$, respectively, while the elasticity operator $\mathbb{A}$ is given by (6a). In that purpose, let us consider the transition between an elastic solid to a damaged elastoviscoplastic material that undergoes permanent deformations. A simple test-case is considered: the uniaxial compression of a rectangular sample of an initially undamaged elastic solid. This benchmark permits to point out both the pre- and post-failure behavior of materials. Since the flow is slow, inertia terms could be neglected. Since deformations are small, convective 
and tensor upper derivative terms are also neglected. See Appendix B.1 for the complete problem statement after these simplifications.

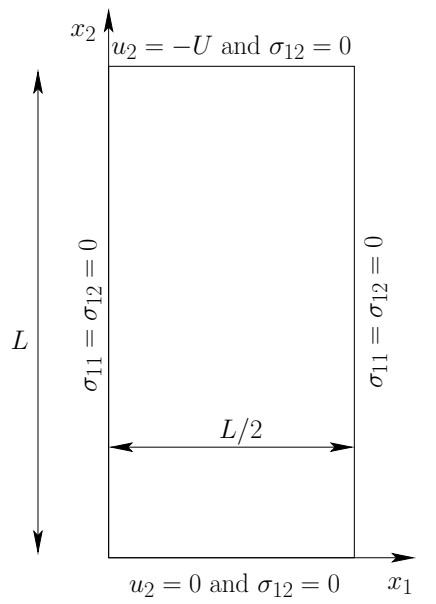

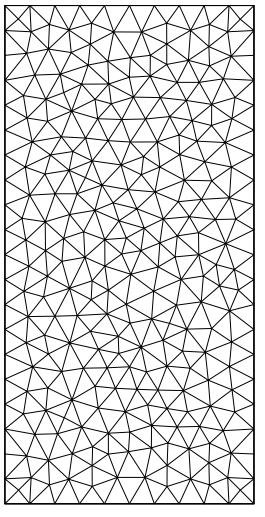

$$
h=L / 20
$$

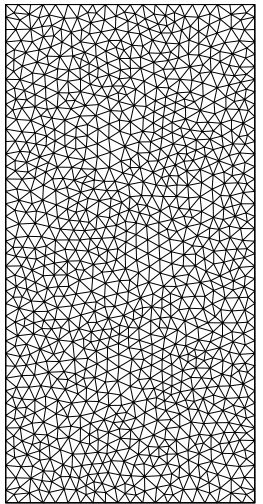

$h=L / 40$

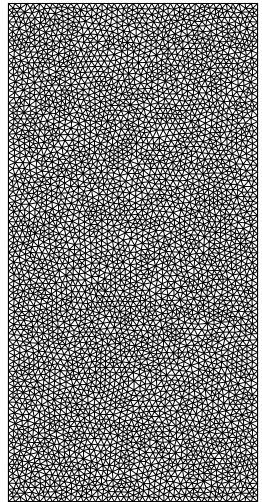

$h=L / 80$

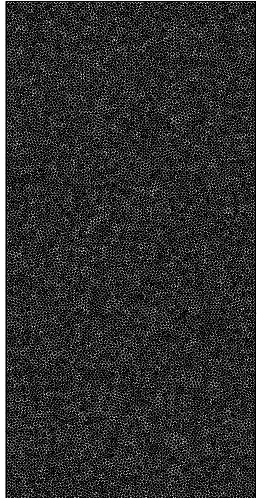

$h=L / 160$

Figure 5: (left) The uniaxial compression experiment. (right) Family of quasi-uniform unstructured meshes.

The horizontal dimensions of the sample are taken much larger than its thickness, hence the problem could be considered as two-dimensional. Let $\Omega=] 0, L / 2[\times] 0, L[$ be the computational domain where $L>0$ is the characteristic length (see Fig. 5.left) and $\left(0, x_{1}, x_{2}\right)$ denotes the Cartesian coordinate system. Compression is applied by prescribing at any time $t>0$ a constant vertical velocity, $-U$, where $U>0$, on the top edge of the plate, while, on the bottom edge, the plate is maintained by imposing a vertical velocity to zero and no confinement is applied on the lateral edges:

$$
\begin{aligned}
u_{2}\left(t, x_{1}, L\right) & =-U \text { and } \sigma_{12}\left(t, x_{1}, L\right)=0, \quad \forall x_{1} \in[0, L / 2] \\
u_{2}\left(t, x_{1}, 0\right) & =0 \text { and } \sigma_{12}\left(t, x_{1}, 0\right)=0, \quad \forall x_{1} \in[0, L / 2] \\
\sigma_{11}\left(t, x_{1}, x_{2}\right) & =\sigma_{12}\left(t, x_{1}, x_{2}\right)=0, \quad \forall x_{1} \in\{0, L / 2\}, x_{2} \in[0, L]
\end{aligned}
$$

The initial conditions for both the elastic deformation $\gamma_{e}$ and the damage $d$ are zero.

\begin{tabular}{|c|c|c|}
\hline parameter & value & dimension \\
\hline \hline$L$ & $200 \times 10^{3}$ & $\mathrm{~m}$ \\
\hline$U$ & $2 \times 10^{-3}$ & $\mathrm{~m} . \mathrm{s}^{-1}$ \\
\hline$E_{0}$ & $28 \times 10^{6}$ & $\mathrm{~Pa}$ \\
\hline$\sigma_{y 0}$ & $50 \times 10^{3}$ & $\mathrm{~Pa}$ \\
\hline$\sigma_{c}$ & $56 \times 10^{3}$ & $\mathrm{~Pa}$ \\
\hline$\eta_{0}$ & $1.4 \times 10^{12}$ & Pa.s \\
\hline$\eta_{s}$ & $1.4 \times 10^{8}$ & Pa.s \\
\hline$\eta_{d}$ & $2.8 \times 10^{8}$ & Pa.s \\
\hline
\end{tabular}

\begin{tabular}{|c|c|c|}
\hline number & \multicolumn{1}{|c|}{ value } & expression \\
\hline \hline$W e$ & $5 \times 10^{-4}$ & $U\left(\eta_{s}+\eta_{0}\right) /\left(L E_{0}\right)$ \\
\hline$W e_{d}$ & $10^{-7}$ & $U \eta_{d} /\left(L E_{0}\right)$ \\
\hline$\gamma_{y}$ & $1.8 \times 10^{-3}$ & $\sigma_{y 0} / E_{0}$ \\
\hline$\gamma_{c}$ & $2 \times 10^{-3}$ & $\sigma_{c} / E_{0}$ \\
\hline$\nu_{0}$ & 0.30 & \\
\hline$\nu_{1}$ & 0.49 & \\
\hline$\mu$ & 0.7 & \\
\hline $1-\alpha$ & $10^{-4}$ & $\eta_{s} /\left(\eta_{s}+\eta_{0}\right)$ \\
\hline
\end{tabular}

Table 2: Table of physical dimensional (left) and dimensionless (right) parameters.

Recall that the practical choices for $\mathbb{A}, \phi_{p}$ and $\phi_{d}$ are given by (6k), (6e) and (6k), respectively. A dimensionless analysis (see Appendix B.2) shows that there are eight dimensionless numbers involved in the problem: $W e, W e_{d}, \gamma_{y}, \gamma_{c}, \nu_{0}, \nu_{1}, \mu$ and $\alpha$, given in Table 2 together with practical values for the dimensional parameters. Dimensional values are chosen in order to be representative of a natural quasi-brittle material (rock or ice). Indeed, such large values for $L$ 
and $U$ correspond to geophysical applications. Values for both the friction coefficient $\mu$ and the damage yield stress parameter $\sigma_{c}$ base on experimental measurements on the Baltimore in-situ sea ice station presented on Fig. 2. The viscoplastic cohesion $\sigma_{y 0}$ is about $10 \%$ smaller than $\sigma_{c}$ in order for the viscoplastic cone $T_{\mu, \sigma_{y}}$ to be included in the damage one $T_{\mu, \sigma_{c}}$. The elastic modulus $E_{0}$ and the Poisson ratio $\nu_{0}$ are also representative of some undamaged rocks and ice, while $\nu_{1}$, associated to a fully damaged one, is close to $1 / 2$. See [53] for a thorough discussion about the variation of the Poisson ratio versus damage. The characteristic time for damage propagation $\eta_{d} / E_{0}$ is much smaller than both the time scale $L / U$ of loading and the time scale $\eta_{0} / E_{0}$ of viscoelasticity and the viscosities $\eta_{0}$ and $\eta_{d}$ are chosen accordingly. This large separation of scales ensures quasi-static conditions for damage. Finally, note that the fully damaged material $(d=1)$ is represented here by a Newtonian fluid with a very weak viscosity $\eta_{s}$, i.e. $\eta_{s}$ is small compared to $\eta_{0}$.

Observe that the geometry of the material is symmetric: this uniaxial compression problem admits several solutions, e.g. left- or right- shaped solutions. Conversely, asymmetric geometries or the presence or material heterogeneities suppress this indetermination. A typical example of such non-unicity of the solution is the buckling of a beam. Here, both the viscoplastic $\sigma_{y 0}$ cohesion and its damage counterpart $\sigma_{c}$ are assumed to contain an uniform random spatial heterogeneity of $30 \%$ around their respective mean values. The final computational time $t_{f}$ is chosen sufficiently large for the post-failure to be reached: $(U / L) t_{f}=3 \times 10^{-2}$.

\subsection{Space and time discretizations}

\begin{tabular}{|l|r|r|r|}
\hline$h / L$ & \# elements & $(U / L) \Delta t$ & \# time steps \\
\hline \hline $1 / 20$ & 508 & $1.2 \times 10^{-5}$ & 2500 \\
\hline $1 / 40$ & 2064 & $0.6 \times 10^{-5}$ & 5000 \\
\hline $1 / 80$ & 8518 & $0.3 \times 10^{-5}$ & 10000 \\
\hline $1 / 160$ & 33858 & $1.5 \times 10^{-6}$ & 20000 \\
\hline
\end{tabular}

Table 3: Table of numerical parameters.

The flow domain is discretized by a family of quasi-uniform unstructured meshes composed of triangular elements and generated by the gmsh mesh generator [19]. The characteristic mesh size is denoted as $h: L / h$ is the number of elements along the vertical edge of the dimensionless computational domain. Computations are performed with both $h=L / 20, L / 40, L / 80$ and $L / 160$ (see Fig. 5.right). The time step $\Delta t$ is chosen in order to solve the smallest dimensionless time scale associated to damage. For the first mesh, associated with $h=L / 20$, we choose $(U / L) \Delta t=1.2 \times 10^{-5}$. Then, the time step is divided by two for each mesh refinement, as shown on Table 3 . The system of equation is then solved by a numerical algorithm postponed in Appendix B and implemented by using the Rheolef finite elements [52]. Note that the mesh size grows by a factor four at each mesh refinement while the number of time steps grows by a factor two. Thus, the computing time is expected to grow at least by a factor eight at each refinement. Indeed, the resolution on a workstation running ten CPUs (Intel-9 at $3.70 \mathrm{GHz}$ ) requires about $7 \mathrm{mn}$ on the first mesh and about 70 hours for the fourth one: the effective growth factor of computing time for each refinement is of about 8.4 .

\subsection{Interpretation of the solutions}

Recall that the dissipation splits as $w=w_{p}+w_{d}$, where $w_{p}$ and $w_{d}$ are its two positive contributions, associated respectively to viscoplasticity and damage and defined by (4a)-(4b). Fig. 6 plots the averaged value of these two terms during all the process. The averaged quantities, denoted as $\bar{w}_{p}$ and $\bar{w}_{d}$, are simply defined by

$$
\bar{w}_{\beta}(t)=\frac{1}{\operatorname{meas}(\Omega)} \int_{\Omega} w_{\beta}(t, \boldsymbol{x}) \mathrm{d} x, \quad \beta \in\{p, d\}
$$


For convenience, a dimensionless time $\gamma=(U / L) t$ is introduced. Note that, in the context of the present uniaxial compression benchmark, $\gamma$ interprets as the amplitude of the deformation on the top boundary, which justifies the notation. Also $\eta_{t o t}=\eta_{s}+\eta_{0}$ denotes the total viscosity. Observe first on Fig. 6 that $w_{p}$ and $w_{d}$ present similar global variations, while the maximums differ by about one order of magnitude. Let us study with details the different flow regimes.

i) For small times, the elastic stress $\sigma_{e}$ is still small, it belongs to the translated DruckerPrager cone $T_{\mu, \sigma_{y}}$. Thus, the viscoplastic deformation rate $\dot{\gamma}_{p}$ is zero (Fig. 6.bottom-left) and, from (1), we get $\dot{\gamma}=\dot{\gamma}_{e}$. Then, from its definition (4a), the viscous dissipation $w_{p}$ reduces to bulk viscous effects i.e. $w_{p}=2 \eta_{s}|\dot{\gamma}|^{2}$, which is small, as shown on Fig. 6.top-left, since both $\eta_{s}$ and $\dot{\gamma}$ are small. Next, observe on Fig. 6.top-right that $w_{d}=0$. Indeed, since $\sigma_{y}<\sigma_{c}$, we have $\boldsymbol{\sigma}_{e} \in T_{\mu, \sigma_{y}} \subset T_{\mu, \sigma_{c}}$ and then damage is not yet able to develop. It means that, during this first regime, the material behaves as an undamaged solid KelvinVoigt viscoelastic one. Moreover, since the bulk viscosity $\eta_{s}$ is small, the behavior is close to those of a pure solid elastic and undamaged material: this is the quasi-elastic flow regime.

ii) This first regime stops at the dimensionless time $\gamma_{1} \approx 5.16 \times 10^{-3}$ when a first plastic even appears and a viscoplastic deformation $\dot{\gamma}_{p}$ starts to develop. Indeed, the elastic stress $\sigma_{e}$ goes outside of the translated Drucker-Prager cone $T_{\mu, \sigma_{y}}$. Note that, during this second flow regime, $\boldsymbol{\sigma}_{e}$ still lives inside second translated Drucker-Prager cone $T_{\mu, \sigma_{c}}$ associated to damage. Recall that $\sigma_{c}>\sigma_{y}$ and then $w_{p}=0$ i.e. the material is still undamaged. This regime stops at $\gamma_{2} \approx 5.73 \times 10^{-3}$ when a first damage even appears (Fig. 6.top-right). Here, $\sigma_{c}$ is only $10 \%$ larger than $\sigma_{y}$ and then, this second flow regime is short. Since $\dot{\gamma}_{p} \neq 0$ the material behaves as an undamaged elastoviscoplastic one. Both the two quasi-elastic and elastoviscoplastic flow regimes correspond to a global pre-failure regime.

iii) The third flow regime starts at $\gamma_{2}$, when a first damage even appears. Now, the elastic stress $\sigma_{e}$ lives outside of the second translated Drucker-Prager cone $T_{\mu, \sigma_{c}}$. Observe on Fig. 6 the fast grown of both the damage dissipation $w_{d}$ and the damage rate $\dot{d}$. Both viscoplastic $w_{p}$ and damage $w_{d}$ dissipation grow in this third flow regime: this is the failure regime.

iv) The fourth and last flow regime starts at $\gamma_{3} \approx 1.83 \times 10^{-2}$ when both $w_{d}$ and $w_{p}$ reach a maximum and start a fast decrease: this is the post-failure regime.

After this interpretation of the process in terms of global flow regimes, let us turn to a local representation of the solution. Fig. 7 shows the isocontours of the damage $d$ at $\gamma_{3}$, when the damage rate is maximum, and of the deformation rate $|\dot{\gamma}|$ at the end of the process $(\gamma=0.03)$. Observe the correspondence of the localization for these two quantities: damage develops where stress and deformation are important and, correspondingly, the deformation rate localizes in damaged regions. For all the four meshes, the corresponding numerical solution are represented.

First, observe that the damage $d$ develops a dense network of fracture at all scales: the finer the mesh is, the denser the fracture network appears, with new small scales features. As a result, the network of fracture presents a fractal-like pattern. See [8, p. 1354] for a thorough analysis of scale invariance for such damage processes. Simultaneously, observe on the $|\dot{\gamma}|$ maps that the deformation rate localizes and tends to collapse on few main fractures that enforce the existing damage in a coupled mechanism. Second, observe that the main localization of the deformation rate $\dot{\gamma}$ develops from top-right to bottom-left for the second mesh $h=L / 40$, while solutions based on other meshes present an alternative direction. The mesh-based space discretization introduces an additional heterogeneity that induces a different direction for the main failure. Recall that the behavior of approximate solutions depends upon all local heterogeneities, including the mesh discretization. Third, observe on Fig. 7.right the direction of the localization for both the damage and the deformation: the present model predicts an angle of about $30^{\circ}$ with the vertical axis, which is in good agreement with experimental observations (see e.g. [29], pages 76 and 88). This 
could be considered as an improvement of a previous work on the MEB model, where an angle of about $40^{\circ}$ was predicted (see [8], Fig. 5 p. 1352).

The behavior of the material on the top boundary, where the normal velocity is imposed, is of major importance: the normal stress component $\sigma_{y y}$ on the top boundary of the domain is averaged as

$$
\bar{\sigma}_{n}(t)=\frac{2}{L} \int_{x=0}^{L / 2} \sigma_{22}\left(t, x_{1}, L\right) \mathrm{d} x_{1}
$$

Fig. 8.left shows that $\sigma_{n}$ grows linearly during the first regime, as expected. In the second flow regime, the stress continues to increase, and reaches a maximum in the third flow regime. The last regime is related to a fast decrease of the normal stress: cracks collapse and the deformation localizes on global directions that cross all the domain.

Finally, Fig. 8.right shows the deformed geometry at the end of the process $(\gamma=0.03)$ together with the isoline separating the fluid and solid regions. Recall that, in the solid regions, the material is not rigid: it behaves as a Kelvin-Voigt viscoelastic solid. Observe that the geometry is now spitted in several bricks separated by cracks. The grayscale represents the norm $|\dot{\gamma}|$ of the deformation rate.

The results presented on Figs. 6, 7 and 8.left show a convergence versus mesh refinement that looks random to a certain extent, e.g. for the location of fluid regions during the last flow regime. Recall that the geometry of the material is symmetric: this uniaxial compression problem admits several solutions, e.g. left- or right- shaped solutions and a typical similar problem is the buckling of a beam. The introduction of material heterogeneities suppresses this indetermination: here, both the viscoplastic $\sigma_{y 0}$ cohesion and its damage counterpart $\sigma_{c}$ are assumed to contain an uniform random spatial heterogeneity of $30 \%$ around their respective mean values. Finally, note that others kind of data heterogeneities could be introduced similarly: instead of $\sigma_{y 0}$ and $\sigma_{c}$ cohesions, we could consider varying e.g. boundary conditions. The unstructured mesh-based discretization also introduces an additional kind of heterogeneity. Observe first that global quantities such as those shown on Fig. 6 clearly converge with mesh refinement until time $\gamma_{3}$ : during the last flow regime, Figs. 6 and 8.left show that the behavior is only qualitatively similar when changing the mesh. Indeed, during the last flow regime, the process is characterized by the formation of local bricks separated by cracks, as shown on Fig. 8.right, and these features are sensible to the local heterogeneities of both $\sigma_{y 0}$ and $\sigma_{c}$. Nevertheless, some mesoscale features, such as the $30^{\circ}$ angle for the direction of the localization for both the damage and the deformation, appear to be robust with mesh refinement, as shown on Fig. 7.

\section{Conclusion and perspectives}

A new brittle-elastoviscoplastic (BEVP) fluid model is presented in this paper. This model is relatively simple to use, as it contains few material parameters and a simple fixed-point algorithm is effective for solving the coupled system of equations. The model combines some existing fundamental features such as elasticity, plasticity and brittle damage. The combination of them bases on thermodynamics that ensures the positivity of the dissipation and the Onsager symmetry. Moreover, thermodynamics points out the link between thixotropy and damage in the context of elastoviscoplastic (EVP) fluids. Theoretical results on the Drucker-Prager plasticity criterion are completed in order to use it for general EVP fluids. Preliminary results with the proposed BEVP model are very encouraging. Indeed, the model allows both the representation of the pre-failure, failure and post-failure behavior of quasi-brittle materials. Future works will consider applications in geosciences, namely sea ice flows and earthquake modeling. Finally, the new theoretical results on Drucker-Prager plasticity presented in this paper open new paths for the mathematical modeling of a large class of materials that presents microstructure of particles with frictional contacts 

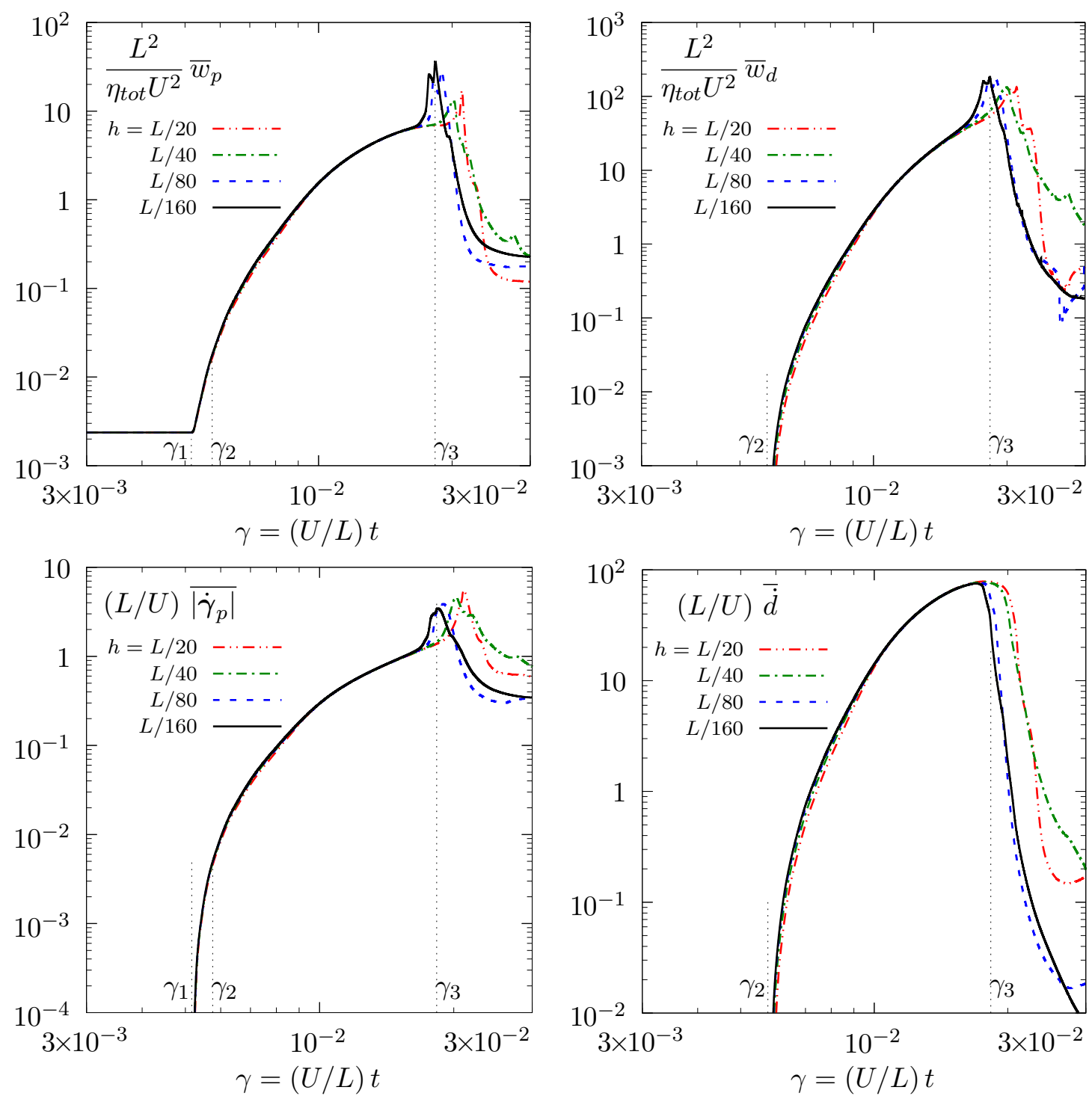

Figure 6: Averaged value of (top-left) the viscoplastic dissipation $w_{p} ;$ (top-right) the damage dissipation $w_{d}$; (bottom-left) the viscoplastic deformation rate $\left|\dot{\gamma}_{p}\right| ;$ (bottom-right) the damage rate. 

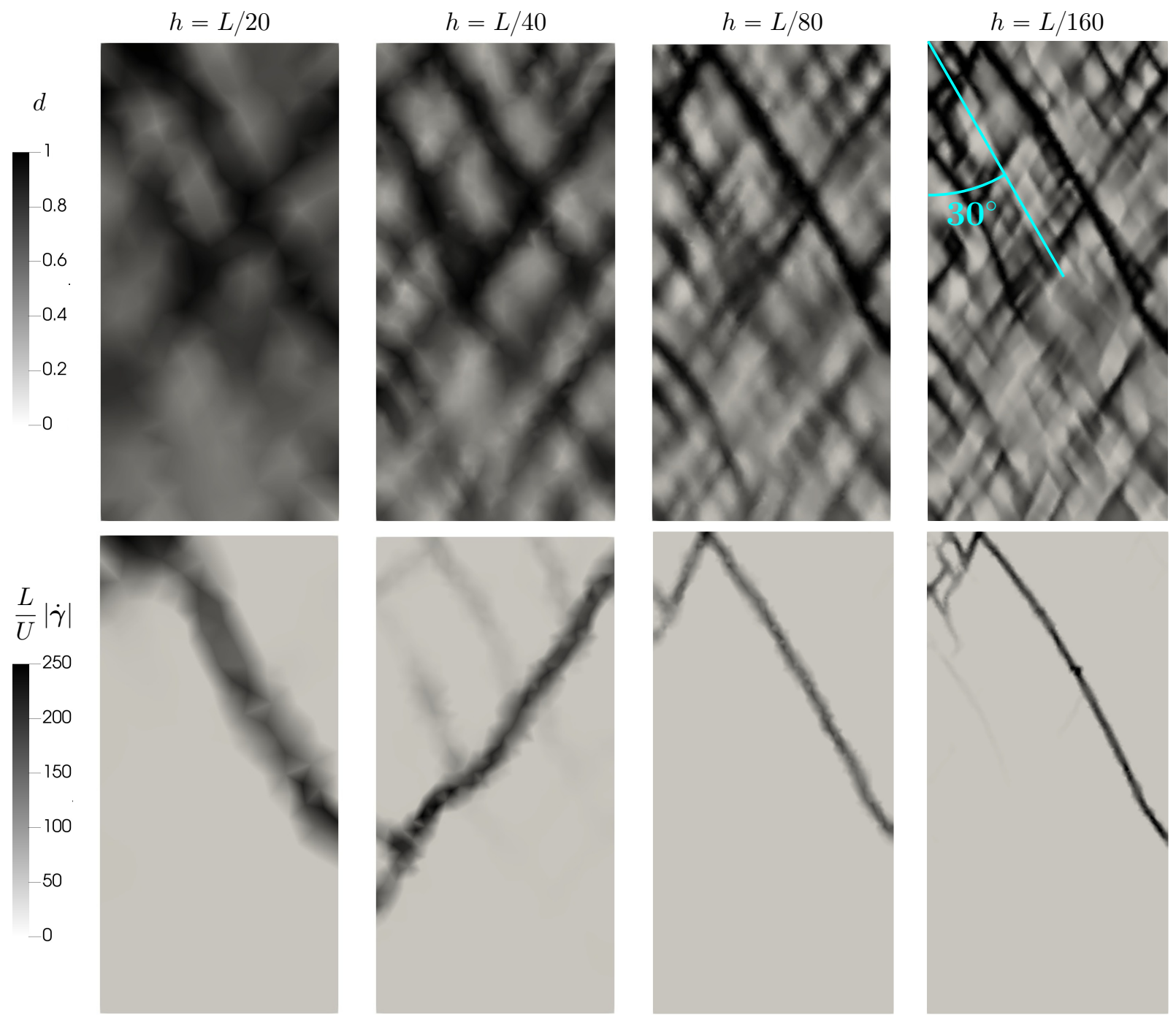

Figure 7: Isocontours of the solution for increasing mesh resolution: (top) damage $d$ at the end of the failure regime $\left(\gamma=\gamma_{3}\right)$; (bottom) norm of the deformation rate $|\dot{\gamma}|$ at the end of the process $(\gamma=0.03)$. 

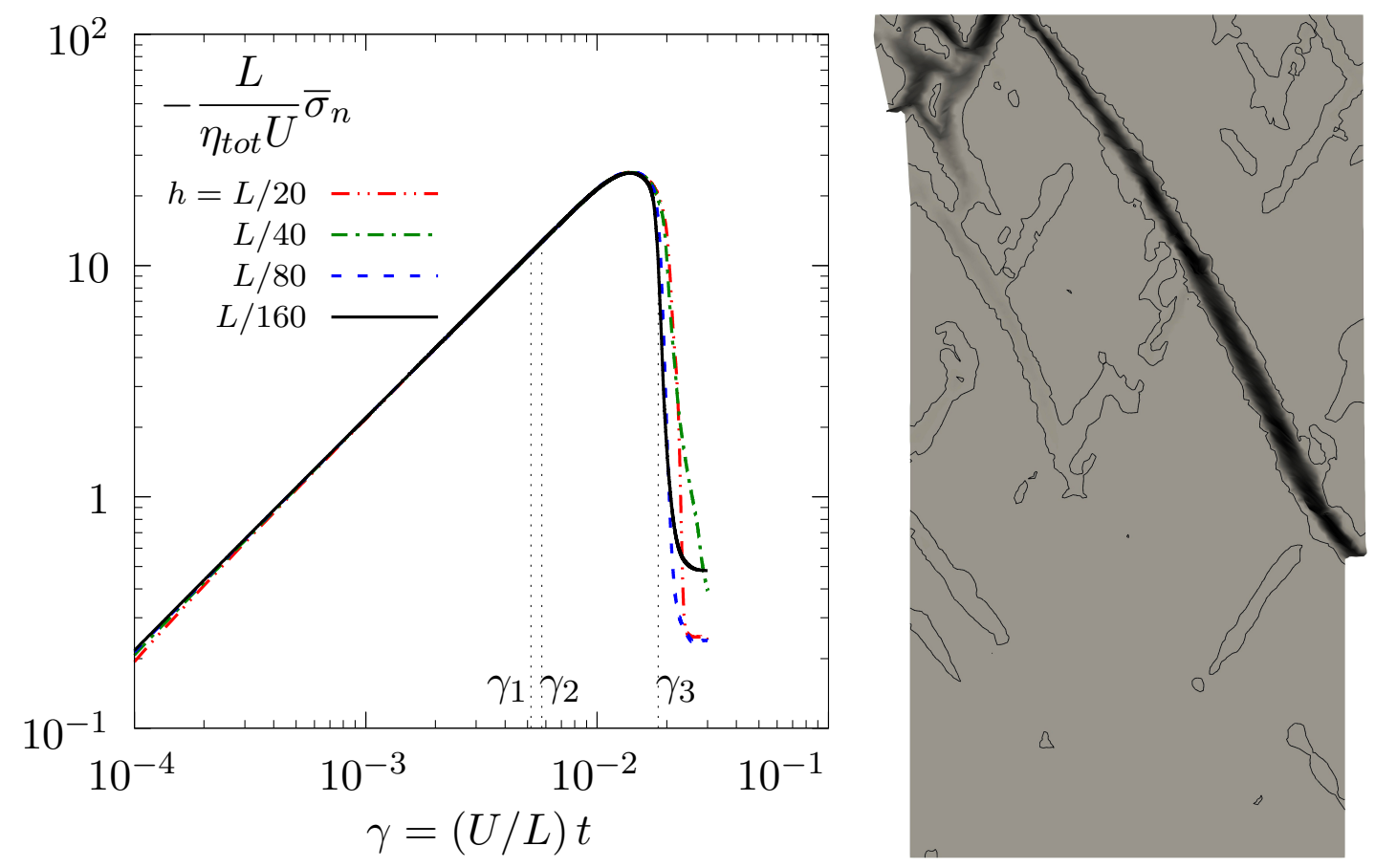

Figure 8: (left) Averaged value of normal stress on the top boundary ; (right) On the deformed geometry, separation line of the solid/fluid together with isocontours of $|\dot{\gamma}|$ at the end of the process $(\gamma=0.03)$. 
and large scale rearrangements, e.g. dry or wet granular flows, concentrated suspensions and rocks and cements undergoing large and unbounded deformations.

Acknowledgments - A preliminary version of this work was presented at the 8th viscoplastic fluid workshop at Cambridge in 2019. The author would like to thank all the participants for very stimulating discussions. The author would also thank Jérôme Weiss and Véronique Dansereau for thorough discussions about potential applications to sea ice modeling and Gilles Daviet for fruitful discussions on optimization methods applied to the Drucker-Prager criterion.

\section{References}

[1] L. R. Alejano and A. Bobet. Drucker-Prager criterion. Rock Mech. Rock Eng., 45(6):995-999, 2012 .

[2] D. Amitrano, J.-R. Grasso, and D. Hantz. From diffuse to localised damage through elastic interaction. Geophys. Res. Lett., 26:2109-2112, 1999.

[3] T. Barker, D. G. Schaeffer, P. Bohorquez, and J. M. N. T. Gray. Well-posed and ill-posed behaviour of the $\mu(I)$-rheology for granular flows. J. Fluid Mech., 779:794-818, 2015.

[4] H. A. Barnes. Thixotropy - a review. J. Non-Newt. Fluid Mech., 70(1):1-33, 1997.

[5] F. Bautista, J. M. de Santos, J. E. Puig, and O. Manero. Understanding thixotropic and antithixotropic behavior of viscoelastic micellar solutions and liquid crystalline dispersions. I. The model. J. Non-Newt. Fluid Mech., 80(2-3):93-113, 1999.

[6] A. N. Beris and B. J. Edwards. Thermodynamics of flowing systems with internal microstructure. Oxford University Press, UK, 1994.

[7] P. P. Bhat, M. Pasquali, and O. A. Basaran. Beads-on-string formation during filament pinch-off: dynamics with the PTT model for non-affine motion. J. Non-Newt. Fluid Mech., 159(1-3):64-71, 2009.

[8] V. Dansereau, J. Weiss, P. Saramito, and P. Lattes. A Maxwell-elasto-brittle rheology for sea ice modelling. Cryosphere, 10:1339-1359, 2016.

[9] V. Dansereau, J. Weiss, P. Saramito, P. Lattes, and E. Coche. Ice bridges and ridges in the Maxwell-EB sea ice rheology. The Cryosphere, 11:2033-2058, 2017.

[10] G. Daviet. Modèles et algorithmes pour la simulation du contact frottant dans les matériaux complexes. Application aux milieux fibreux et granulaires. $\mathrm{PhD}$ thesis, Université de Grenoble, 2016 .

[11] G. Daviet and F. Bertails-Descoubes. Nonsmooth simulation of dense granular flows with pressure-dependent yield stress. J. Non-Newt. Fluid Mech., 234:15-35, 2016.

[12] P. R. de Souza Mendes. Modeling the thixotropic behavior of structured fluids. J. Non-Newt. Fluid Mech., 164:66-75, 2009.

[13] C. J. Dimitriou and G. H. McKinley. A comprehensive constitutive law for waxy crude oil: a thixotropic yield stress fluid. Soft Matt., 10(35):6619-6644, 2014.

[14] D. C. Drucker and W. Prager. Soil mechanics and plastic analysis or limit design. Quart. Appl. Math., 10(2):157-165, 1952.

[15] I. Ekeland and R. Temam. Convex analysis and variational problems. SIAM, Philadelphia, USA, 1999. 
[16] F. Erdogan and G. C. Sih. On the crack extension in plates under plane loading and transverse shear. J. Basic Eng., 85(4):519-525, 1963.

[17] A. G. Fredrickson. A model for the thixotropy of suspensions. AIChe J., 16(3):436-441, 1970.

[18] I. Frigaard and A. Renteria. Stability of flows with the BMP model in the yield stress limit. Korea-Australia Rheology J., 31(4):211-228, 2019.

[19] C. Geuzaine and J.-F. Remacle. Gmsh reference manual, 2021. http://geuz.org/gmsh/ doc/texinfo/gmsh.pdf.

[20] R. J. Gordon and W. R. Schowalter. Anisotropic fluid theory: a different approach to the dumbbell theory of dilute polymer solutions. J. Rheol., 16:79-97, 1972.

[21] A. A. Griffith. The phenomena of rupture and flow in solids. Phil. Trans. R. Soc. Lond. A, 221(582-593):163-198, 1921.

[22] M. E. Gurtin, E. Fried, and L. Anand. The mechanics and thermodynamics of continua. Cambridge university press, 2010.

[23] B. Halphen and Q. S. NGuyen. Sur les matériaux standards généralisés. J. Méca., 14:39-63, 1975.

[24] N. R. Hansen and H. L. Schreyer. A thermodynamically consistent framework for theories of elastoplasticity coupled with damage. Int. J. Solids Struct., 31(3):359-389, 1994.

[25] M. J. Heap and D. R. Faulkner. Quantifying the evolution of static elastic properties as crystalline rock approaches failure. Int. J. Rock Mech. Min. Sci., 45(4):564-573, 2008.

[26] M. J. Heap, S. Vinciguerra, and P. G. Meredith. The evolution of elastic moduli with increasing crack damage during cyclic stressing of a basalt from Mt. Etna volcano. Tectonophysics, 471(1-2):153-160, 2009.

[27] M. Hjiaj, J. Fortin, and G. de Saxcé. A complete stress update algorithm for the nonassociated Drucker-Prager model including treatment of the apex. Int. J. Eng. Sci., 41(10):1109-1143, 2003.

[28] M. Hütter, M. Grmela, and H. C. Öttinger. What is behind the plastic strain rate? Rheol. Acta, 48(7):769-778, 2009.

[29] J. C. Jaeger, N. G. W. Cook, and R. Zimmerman. Fundamentals of rock mechanics. Blackwell, fourth edition, 2007.

[30] C. S. Jog. A concise proof of the representation theorem for fourth-order isotropic tensors. J. Elast., 85(2):119-124, 2006.

[31] P. Jop, Y. Forterre, and O. Pouliquen. A constitutive law for dense granular flows. Nature, 441:727-730, 2006.

[32] J. W. Ju. Isotropic and anisotropic damage variables in continuum damage mechanics. $J$. Eng. Mech., 116(12):2764-2770, 1990.

[33] L. M. Kachanov. Foundations of the theory of plasticity. Elsevier, 1971.

[34] F. Kang and S. Zhong-Ci. Mathematical theory of elastic structures. Springer, 1996.

[35] R. G. Larson and Y. Wei. A review of thixotropy and its rheological modeling. J. Rheol., 63(3):477-501, 2019.

[36] J. Lemaitre. A continuous damage mechanics model for ductile fracture. J. Eng. Mat. Tech., 107(1):83-89, 1985. 
[37] J. Lemaitre. A course on damage mechanics. Springer, second edition, 1996.

[38] A. I. Leonov. Nonequilibrium thermodynamics and rheology of viscoelastic polymer media. Rheol. Acta, 15(2):85-98, 1976.

[39] O. Manero, J. H. Pérez-López, J. I. Escalante, J. E. Puig, and F. Bautista. A thermodynamic approach to rheology of complex fluids: the generalized BMP model. J. Non-Newt. Fluid Mech., 146(1-3):22-29, 2007.

[40] G. A. Maugin. The thermomechanics of plasticity and fracture. Cambridge University Press, UK, 1992.

[41] J. C. Maxwell. On the dynamical theory of gases. Phil. Trans. R. Soc. Lond., 157:49-88, 1867.

[42] J. Mewis and N. J. Wagner. Thixotropy. Adv. Colloid Interf. Sci., 147:214-227, 2009.

[43] J.-J. Moreau. Fonctionnelles convexes. Séminaire Jean Leray, 2:1-108, 1966. https://eudml. org/doc/112529.

[44] S. Murakami. Continuum damage mechanics: a continuum mechanics approach to the analysis of damage and fracture. Springer, 2012.

[45] J. G. Oldroyd. Rectilinear flow of non-Bingham plastic solids and non-Newtonian viscous liquids. II. Math. Proc. Cambridge Phil. Soc., 47(02):410-418, 1951.

[46] N. Phan-Thien, M. Newberry, and R. I. Tanner. Non-linear oscillatory flow of a soft solid-like viscoelastic material. J. Non-Newt. Fluid Mech., 92(1):67-80, 2000.

[47] R. T. Rockafellar. Convex analysis. Princeton university press, NJ, USA, 1970.

[48] P. Saramito. Efficient simulation of nonlinear viscoelastic fluid flows. J. Non Newt. Fluid Mech., 60:199-223, 1995.

[49] P. Saramito. A new constitutive equation for elastoviscoplastic fluid flows. J. Non-Newt. Fluid Mech., 145(1):1-14, 2007.

[50] P. Saramito. A new elastoviscoplastic model based on the Herschel-Bulkley viscoplasticity. J. Non-Newt. Fluid Mech., 158(1-3):154-161, 2009.

[51] P. Saramito. Complex fluids: modelling and algorithms. Springer, 2016.

[52] P. Saramito. Efficient C++ finite element computing with Rheolef. CNRS and LJK, 2018. http://cel.archives-ouvertes.fr/cel-00573970.

[53] P. Saramito, V. Dansereau, and J. Weiss. Linking bulk modulus to an unilateral damage yield criterion: a thermodynamic modeling approach. to appear, 2021.

[54] P. Saramito and A. Wachs. Progress in numerical simulation of yield stress fluid flows. J. Rheol., 56(3):211-230, 2017.

[55] C. Tang. Numerical simulation of progressive rock failure and associated seismicity. Int. J. Rock Mech. Min. Sci., 34(2):249-261, 1997.

[56] J. Weiss and E. M. Schulson. Coulombic faulting from the grain scale to the geophysical scale: lessons from ice. J. Phys. D, 42(21):214017, 2009.

[57] S. Zapperi, A. Vespignani, and H. E. Stanley. Plasticity and avalanche behaviour in microfracturing phenomena. Nature, 388:658-660, 1997. 


\section{Appendix A. Convex analysis applied to viscoplasticity}

The aim of this appendix is to present the complete proof of theorem 2, page 2, in a self-contained way. This appendix starts with several well-known results from convex analysis due to Rockafellar [47] and Moreau [43]. Next, new results are established concerning von Mises and DruckerPrager plasticity criteria. These new results extend previous works from Saxcé and coworkers [27] and Daviet [10, app. A] on Drucker-Prager plasticity.

\section{Appendix A.1. Notations and definitions}

Let $N \geqslant 1$ be the physical space dimension. The space of symmetric real $N \times N$ matrix is denoted by $\mathbb{R}_{s}^{N \times N}$. Its scalar product is $\boldsymbol{\delta}: \boldsymbol{\tau}$ for all $\boldsymbol{\delta}, \boldsymbol{\tau} \in \mathbb{R}_{s}^{N \times N}$ and $|\boldsymbol{\delta}|=(\boldsymbol{\delta}: \boldsymbol{\delta})^{\frac{1}{2}}$ is the associated norm.

Definition 4 (convex set).

$A$ set $C \subset \mathbb{R}^{N \times N}$ is convex when

$$
\theta \boldsymbol{\delta}_{1}+(1-\theta) \boldsymbol{\delta}_{2} \in C, \quad \forall \boldsymbol{\delta}_{1}, \boldsymbol{\delta}_{2} \in C, \quad \forall \theta \in[0,1]
$$

The set $C$ is strictly convex if and only if

$$
\left.\theta \boldsymbol{\delta}_{1}+(1-\theta) \boldsymbol{\delta}_{2} \in \operatorname{int}(C), \quad \forall \boldsymbol{\delta}_{1}, \boldsymbol{\delta}_{2} \in C, \quad \boldsymbol{\delta}_{1} \neq \boldsymbol{\delta}_{2}, \quad \forall \theta \in\right] 0,1[
$$

where $\operatorname{int}(S)=S \backslash \partial S$ denotes the interior of $S$.

Definition 5 (convex function).

A function $\varphi: \mathbb{R}^{N \times N} \rightarrow \overline{\mathbb{R}}$ is convex if and only if

$$
\varphi\left(\theta \boldsymbol{\delta}_{1}+(1-\theta) \boldsymbol{\delta}_{2}\right) \leqslant \theta \varphi\left(\boldsymbol{\delta}_{1}\right)+(1-\theta) \varphi\left(\boldsymbol{\delta}_{2}\right), \quad \forall \boldsymbol{\delta}_{1}, \boldsymbol{\delta}_{2} \in \mathbb{R}^{N \times N}, \quad \forall \theta \in[0,1]
$$

The function $\varphi$ is strictly convex if and only if

$$
\left.\varphi\left(\theta \boldsymbol{\delta}_{1}+(1-\theta) \boldsymbol{\delta}_{2}\right)<\theta \varphi\left(\boldsymbol{\delta}_{1}\right)+(1-\theta) \varphi\left(\boldsymbol{\delta}_{2}\right), \quad \forall \boldsymbol{\delta}_{1}, \boldsymbol{\delta}_{2} \in \mathbb{R}^{N \times N}, \quad \boldsymbol{\delta}_{1} \neq \boldsymbol{\delta}_{2}, \quad \forall \theta \in\right] 0,1[
$$

Definition 6 (proper function).

For any function $\varphi: \mathbb{R}^{N \times N} \rightarrow \overline{\mathbb{R}}$, let

$$
\operatorname{dom}(\varphi)=\left\{\boldsymbol{\delta} \in \mathbb{R}^{N \times N} / \varphi(\boldsymbol{\delta})<\infty\right\}
$$

Then, $\varphi$ is said to be proper if and only if

$$
\operatorname{dom}(\varphi) \neq \varnothing \quad \text { and } \varphi(\boldsymbol{\delta}) \neq-\infty, \delta \in \mathbb{R}^{N \times N}
$$

Definition 7 (closed function).

For any function $\varphi: \mathbb{R}^{N \times N} \rightarrow \overline{\mathbb{R}}$, let us introduce the epigraph of $\varphi$, defined by

$$
\operatorname{epi}(\varphi)=\left\{(\boldsymbol{\delta}, z) \in \mathbb{R}^{N \times N} \times \mathbb{R} / \varphi(\boldsymbol{\delta}) \leqslant z\right\}
$$

Then, $\varphi$ is said to be closed if and only if the set epi $(\varphi)$ is closed.

Definition 8 (lower semi-continuous function).

A function $\varphi: \mathbb{R}^{N \times N} \rightarrow \overline{\mathbb{R}}$ is said to be lower semi-continuous at $\boldsymbol{\delta}_{0} \in \mathbb{R}^{N \times N}$ if and only if

$$
\forall \varepsilon>0, \exists \alpha>0 \text { such that }\left|\boldsymbol{\delta}-\boldsymbol{\delta}_{0}\right|<\alpha \Rightarrow \varphi(\boldsymbol{\delta}) \geqslant \varphi\left(\boldsymbol{\delta}_{0}\right)-\varepsilon, \quad \forall \boldsymbol{\delta} \in \mathbb{R}^{N \times N}
$$

Proposition 9 (lower semi-continuous).

The following propositions are equivalent

- $\varphi$ is lower semi-continuous 
- $\varphi$ is closed

- the set $\left\{\boldsymbol{\delta} \in \mathbb{R}^{N \times N} / \varphi(\boldsymbol{\delta}) \leqslant z\right\}$ is closed for all $z \in \mathbb{R}$.

Proof: See [43], paragraph 4.a.

Definition 10 (indicator).

Let $C \subset \mathbb{R}_{s}^{N \times N}$. Then, the indicator to $C$, denoted by $\mathscr{I}_{C}$ is defined for all $\boldsymbol{\delta} \in \mathbb{R}_{s}^{N \times N}$ by

$$
\mathscr{I}_{C}(\boldsymbol{\delta})= \begin{cases}0 & \text { when } \boldsymbol{\delta} \in C \\ +\infty & \text { when } \boldsymbol{\delta} \notin C\end{cases}
$$

Proposition 11 (indicator).

$$
\begin{aligned}
\mathscr{I}_{C} \text { is convex } & \Longleftrightarrow C \text { is convex } \\
\mathscr{I}_{C} \text { is proper } & \Longleftrightarrow C \neq \varnothing \\
\mathscr{I}_{C} \text { is closed } & \Longleftrightarrow C \text { is closed }
\end{aligned}
$$

\section{Appendix A.2. Subdifferentials}

Lemma 12 (differentiable convex function). Let $E \subset \mathbb{R}^{N \times N}$ and $\varphi: E \rightarrow \mathbb{R}$ be a differentiable convex function. Then

$$
\varphi\left(\boldsymbol{\delta}_{0}\right)+\frac{\partial \varphi}{\partial \boldsymbol{\delta}}\left(\boldsymbol{\delta}_{0}\right):\left(\boldsymbol{\delta}-\boldsymbol{\delta}_{0}\right) \leqslant \varphi(\boldsymbol{\delta}), \quad \forall \boldsymbol{\delta}, \boldsymbol{\delta}_{0} \in E
$$

Proof: See [15, p. 24], proposition 5.4.
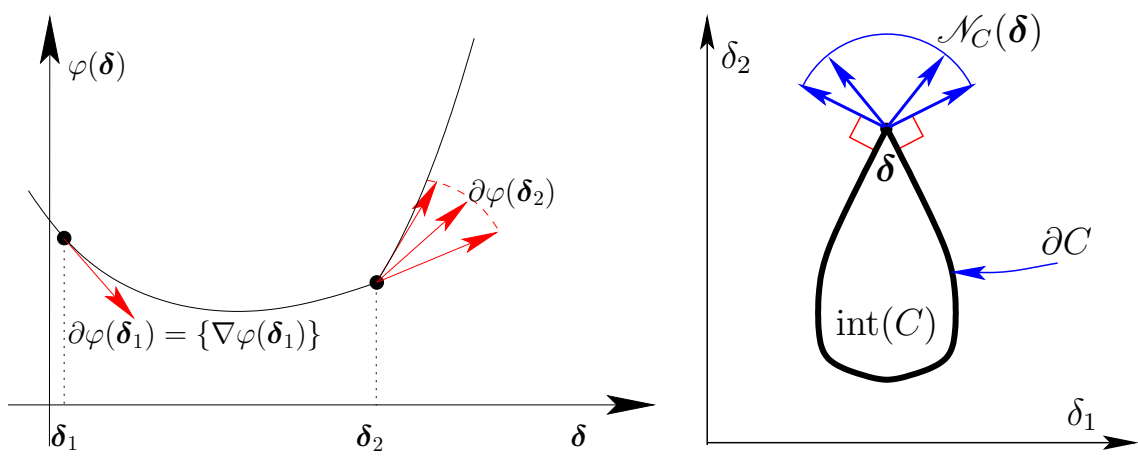

Figure A.9: (left) Subdifferential of a convex function. (right) Normal cone $\mathscr{N}_{C}$ to a convex set $C$.

Definition 13 (subdifferential).

Let $\varphi: \mathbb{R}^{N \times N} \rightarrow \overline{\mathbb{R}}$ be a convex function and $\boldsymbol{\delta}_{0} \in \mathbb{R}^{N \times N}$. If there exists $\boldsymbol{\tau} \in \mathbb{R}^{N \times N}$ such that

$$
\varphi\left(\boldsymbol{\delta}_{0}\right)+\boldsymbol{\tau}:\left(\boldsymbol{\delta}-\boldsymbol{\delta}_{0}\right) \leqslant \varphi(\boldsymbol{\delta}), \quad \forall \boldsymbol{\delta} \in \mathbb{R}^{N \times N}
$$

then $\boldsymbol{\tau}$ is a subdifferential of $\varphi$ at $\boldsymbol{\delta}_{0}$.

The subdifferential at $\boldsymbol{\delta}_{0}$, denoted by $\partial \varphi\left(\boldsymbol{\delta}_{0}\right)$, is the set of all subdifferentials at $\boldsymbol{\delta}_{0}$.

It interprets as a generalization of the usual derivative and it coincides with the convex envelop of all directional derivatives (see Fig. A.9.left). 
Theorem 3 (subdifferential of a sum).

Let $\varphi_{1}, \varphi_{2}: \mathbb{R}^{N \times N} \rightarrow \overline{\mathbb{R}}$ be two convex functions.

If there exists $\boldsymbol{\delta}_{0} \in \operatorname{dom}\left(\varphi_{1}\right) \cap \operatorname{dom}\left(\varphi_{2}\right)$ such that $\varphi_{1}$ is continuous at $\boldsymbol{\delta}_{0}$, then

$$
\partial\left(\varphi_{1}+\varphi_{2}\right)=\partial \varphi_{1}+\partial \varphi_{2}
$$

Proof: See [43], proposition 10.7, page 62. It always holds $\partial\left(\varphi_{1}+\varphi_{2}\right) \subset \partial \varphi_{1}+\partial \varphi_{2}$, but the additional condition is required for the equality to be achieved.

Corollary 14 (subdifferential of sum).

Let $\varphi, \psi: \mathbb{R}^{N \times N} \rightarrow \overline{\mathbb{R}}$ be two convex functions with $\varphi$ proper and closed.

If $\operatorname{int}(\operatorname{dom}(\varphi)) \cap \operatorname{dom}(\psi) \neq \varnothing$ then

$$
\partial(\varphi+\psi)=\partial \varphi+\partial \psi
$$

Proof: From theorem 3.

Corollary 15 (subdifferentials and equality).

Let $\varphi, \psi: \mathbb{R}^{N \times N} \rightarrow \overline{\mathbb{R}}$ be two convex functions such that $\partial \varphi(\delta) \subset \partial \psi(\delta)$ for all $\boldsymbol{\delta} \in \mathbb{R}^{N \times N}$. Then $\varphi$ and $\psi$ differ from a finite constant.

Proof: See [43], paragraph 10.j, page 70.

Proposition 16 (subdifferential and affine map).

Let $\mathbb{F}: \mathbb{R}^{N \times N} \rightarrow \mathbb{R}^{N \times N}$ be and affine map, i.e. $\mathbb{F}(\boldsymbol{\delta})=\mathbb{A}: \boldsymbol{\delta}+\boldsymbol{F}_{*}$, for all $\boldsymbol{\delta} \in \mathbb{R}^{N \times N}$ and where $\mathbb{A}$ is a fourth-order tensor and $\boldsymbol{F}_{*} \in \mathbb{R}^{N \times N}$. Let $\varphi: \mathbb{R}^{N \times N} \rightarrow \mathbb{R}$ be a convex function.

- Then, for all $\boldsymbol{\delta} \in \mathbb{R}^{N \times N}$

$$
\partial(\varphi \circ \mathbb{F})(\boldsymbol{\delta}) \supset \mathbb{A}^{T}: \partial \varphi(\mathbb{F}(\boldsymbol{\delta}))
$$

where $\mathbb{A}^{T}$ denotes the transpose of $\mathbb{A}$.

- Moreover, if $\varphi$ is proper and closed, and if there exists $\boldsymbol{\delta}_{*} \in \operatorname{dom}(\varphi \circ \mathbb{F})$ such that $\varphi$ is continuous at $\mathbb{F}\left(\boldsymbol{\delta}_{*}\right)$, then, for all $\boldsymbol{\delta} \in \mathbb{R}^{N \times N}$

$$
\partial(\varphi \circ \mathbb{F})(\boldsymbol{\delta})=\mathbb{A}^{T}: \partial \varphi(\mathbb{F}(\boldsymbol{\delta}))
$$

Proof: See [10], page 215, property A.12.

Proposition 17 (indicator to deviatoric and spherical matrix). Let us consider the following sets:

$$
\begin{aligned}
\operatorname{ker}(\operatorname{tr}) & =\left\{\boldsymbol{\delta} \in \mathbb{R}^{N \times N} ; \operatorname{tr} \boldsymbol{\delta}=0\right\} \\
\operatorname{ker}(\mathbf{d e v}) & =\left\{\boldsymbol{\tau} \in \mathbb{R}^{N \times N} ; \operatorname{dev} \boldsymbol{\tau}=0\right\}=\{p \boldsymbol{I} ; p \in \mathbb{R}\}
\end{aligned}
$$

Then

$$
\begin{aligned}
& \mathcal{I}_{\mathrm{ker}(\mathrm{tr})}^{*}=\mathcal{I}_{\mathrm{ker}(\text { dev })} \text { and } \mathcal{I}_{\mathrm{ker}(\mathrm{dev})}^{*}=\mathcal{I}_{\mathrm{ker}(\mathrm{tr})} \\
& \partial \mathcal{I}_{\text {ker }(\operatorname{tr})}=\operatorname{ker}(\mathbf{d e v}) \text { and } \partial \mathcal{I}_{\text {ker }(\mathbf{d e v})}=\operatorname{ker}(\operatorname{tr})
\end{aligned}
$$

Proof: Observe first that both $\operatorname{ker}(\operatorname{tr})$ and $\mathcal{I}_{\text {ker }(\mathbf{d e v})}$ are convex cones, as introduced in definition 20. Then, from proposition 23, the conjugate is the indicator to the polar, and from by definition 22 of the polar: $(\operatorname{ker} t r)^{\circ}=\operatorname{ker}(\mathbf{d e v})$ and $(\operatorname{ker} \mathbf{d e v})^{\circ}=\operatorname{ker}(\operatorname{tr})$.

Next, from proposition 26.c, we have $\partial \mathcal{I}_{\text {ker(tr) }}=\mathscr{N}_{\text {ker(tr) }}$ and, from definition 24, we easily check that $\mathscr{N}_{\operatorname{ker}(\operatorname{tr})}=\operatorname{ker}(\mathbf{d e v})$. Finally, the last computation of $\partial \mathcal{I}_{\mathrm{ker}(\mathbf{d e v})}$ is similar. 
Appendix A.3. Convex conjugate

Definition 18 (convex conjugate).

Let $\varphi: \mathbb{R}^{N \times N} \rightarrow \overline{\mathbb{R}}$ be a convex function. The convex conjugate of $\varphi$ also called the Legendre transformation of $\varphi$, denoted by $\varphi^{*}$, is defined for all $\boldsymbol{\tau} \in \mathbb{R}^{N \times N}$ by

$$
\varphi^{*}(\boldsymbol{\tau})=\sup _{\boldsymbol{\delta} \in \mathbb{R}^{N \times N}} \boldsymbol{\delta}: \boldsymbol{\tau}-\varphi(\boldsymbol{\delta})
$$

Proposition 19 (convex conjugate).

The convex conjugate $\varphi^{*}$ of a convex function $\varphi$ is always convex and closed. Moreover, if $\varphi$ is proper and closed, then $\varphi^{* *}=\varphi$ i.e. it is equal to its biconjugate.

Proof: See [43], paragraphs 6.b and 6.d.

Theorem 4 (Fenchel-Young relations).

For any convex function $\varphi: \mathbb{R}^{N \times N} \rightarrow \overline{\mathbb{R}}$, and all $\boldsymbol{\delta}, \boldsymbol{\tau} \in \mathbb{R}^{N \times N}$, we have

$$
\varphi(\boldsymbol{\delta})+\varphi(\boldsymbol{\tau}) \geqslant \boldsymbol{\delta}: \boldsymbol{\tau}
$$

Moreover, if $\varphi$ is proper and closed:

$$
\varphi(\boldsymbol{\delta})+\varphi(\boldsymbol{\tau})=\boldsymbol{\delta}: \boldsymbol{\tau} \Longleftrightarrow \boldsymbol{\tau} \in \partial \varphi(\boldsymbol{\delta}) \Longleftrightarrow \boldsymbol{\delta} \in \partial \varphi^{*}(\boldsymbol{\tau})
$$

Proof: From the definition 18 of the convex conjugate:

$$
\varphi(\boldsymbol{\delta})+\varphi^{*}(\boldsymbol{\tau})=\varphi(\boldsymbol{\delta})+\sup _{\boldsymbol{\mu} \in \mathbb{R}^{N \times N}}(\boldsymbol{\mu}: \boldsymbol{\tau}-\varphi(\boldsymbol{\mu})) \geqslant \varphi(\boldsymbol{\delta})+(\boldsymbol{\delta}: \boldsymbol{\tau}-\varphi(\boldsymbol{\delta}))=\boldsymbol{\delta}: \boldsymbol{\tau}
$$

Then

$$
\begin{aligned}
\varphi(\boldsymbol{\delta})+\varphi^{*}(\boldsymbol{\tau})=\boldsymbol{\delta}: \boldsymbol{\tau} & \Longleftrightarrow \varphi^{*}(\boldsymbol{\tau})=\boldsymbol{\delta}: \boldsymbol{\tau}-\varphi(\boldsymbol{\delta}) \\
& \Longleftrightarrow \boldsymbol{\mu}: \boldsymbol{\tau}-\varphi(\boldsymbol{\mu}) \leqslant \boldsymbol{\delta}: \boldsymbol{\tau}-\varphi(\boldsymbol{\delta}), \quad \forall \boldsymbol{\mu} \in \mathbb{R}^{N \times N} \\
& \Longleftrightarrow \varphi(\boldsymbol{\delta})+(\boldsymbol{\mu}-\boldsymbol{\delta}): \boldsymbol{\tau} \leqslant \varphi(\boldsymbol{\mu}), \quad \forall \boldsymbol{\mu} \in \mathbb{R}^{N \times N} \\
& \Longleftrightarrow \boldsymbol{\tau} \in \partial \varphi(\boldsymbol{\delta})
\end{aligned}
$$

The second part of the equivalence is obtained, when $\varphi$ is proper and closed, by replacing $\varphi$ by $\varphi^{*}$ and using $\varphi^{* *}=\varphi$ from proposition 19 .

Appendix A.4. Convex and normal cones

Definition 20 (convex cone).

$A$ subset $K \subset \mathbb{R}^{N \times N}$ is a convex cone if and only if $a_{1} \boldsymbol{\delta}_{1}+a_{2} \boldsymbol{\delta}_{2} \in K$ for all $\boldsymbol{\delta}_{1}, \boldsymbol{\delta}_{2} \in K$ and $a_{1}, a_{2} \geqslant 0$.

Proposition 21 (convex cone).

Let $K \subset \mathbb{R}^{N \times N}$ be convex cone. Then $0 \in K$ if and only if $K \neq \varnothing$.

Proof: Assume that $K \neq \varnothing$. Then, from the definition of the convex cone and taking zero coefficients, we obtain $0 \in K$.

Definition 22 (polar and dual cones).

For all convex cone $K \subset \mathbb{R}^{N \times N}$, we define the polar cone $K^{\circ}$ and the dual cone $K^{*}$ by

$$
\begin{aligned}
& K^{\circ}=\left\{\boldsymbol{\tau} \in \mathbb{R}^{N \times N} ; \boldsymbol{\delta}: \boldsymbol{\tau} \leqslant 0, \forall \boldsymbol{\delta} \in K\right\} \\
& K^{*}=-K^{\circ}
\end{aligned}
$$


Proposition 23 (convex conjugate of the indicator to a convex cone). Let $K \neq \varnothing$ be a convex cone. Then

$$
\left(\mathcal{I}_{K}\right)^{*}=\mathcal{I}_{K^{\circ}}
$$

Proof: Following the definition 18 of the convex conjugate, we have, for all $\boldsymbol{\delta}, \boldsymbol{\tau} \in \mathbb{R}^{N \times N}$ :

$$
\left(\mathcal{I}_{K}\right)^{*}(\boldsymbol{\tau})=\sup _{\boldsymbol{\delta} \in \mathbb{R}^{N \times N}}\left(\boldsymbol{\delta}: \boldsymbol{\tau}-\mathcal{I}_{K}(\boldsymbol{\delta})\right)=\sup _{\boldsymbol{\delta} \in K} \boldsymbol{\delta}: \boldsymbol{\tau}
$$

As $K$ is a non-empty cone, then $0 \in K$, from proposition 21 , and then, from the previous relation, $\left(\mathcal{I}_{K}\right)^{*}(\boldsymbol{\tau}) \geqslant 0$. Moreover, if $\boldsymbol{\delta}_{0} \in K$ such that $\boldsymbol{\delta}_{0}: \boldsymbol{\tau}>0$, then, for all $\beta \geqslant 0$, we can choose $\alpha=\beta /\left(\boldsymbol{\delta}_{0}: \boldsymbol{\tau}\right)$ and we have both $\left(\alpha \boldsymbol{\delta}_{0}\right): \boldsymbol{\tau}=\beta \geqslant 0$ and $\alpha \boldsymbol{\delta}_{0} \in K$. By taking the supremum on any $\beta \geqslant 0$, we obtain $\left(\mathcal{I}_{K}\right)^{*}(\boldsymbol{\tau})=\infty$. This means

$$
\left(\mathcal{I}_{K}\right)^{*}(\boldsymbol{\tau})=\left\{\begin{array}{ll}
\infty & \text { when } \exists \boldsymbol{\delta}_{0} \in K / \boldsymbol{\delta}_{0}: \boldsymbol{\tau}>0 \\
0 & \text { othewise }
\end{array}\right\}=\left\{\begin{array}{ll}
\infty & \text { when } \boldsymbol{\tau} \in K^{\circ} \\
0 & \text { othewise }
\end{array}\right\}=\mathcal{I}_{K^{\circ}}
$$

from the definition 22 of the polar.

Definition 24 (normal cone).

Let $C \subset \mathbb{R}_{s}^{N \times N}$ be a convex set. Then, the normal cone to $C$, denoted by $\mathscr{N}_{C}$ is defined for all $\boldsymbol{\delta} \in \mathbb{R}_{s}^{N \times N}$ by (see Fig. A.9.right):

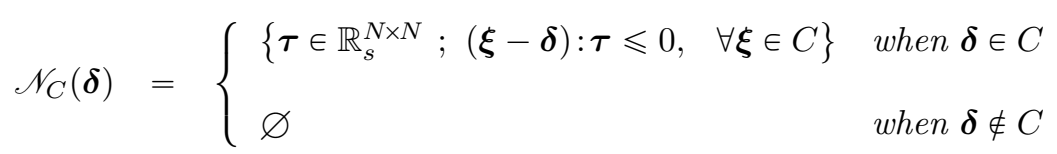

Remark 25 (normal cone).

The name normal cone takes its origin from the fact that it belongs to the class of convex cones, which possesses interesting properties with respect to the convex conjugate.

We can easily check that $\mathscr{N}_{C}(\boldsymbol{\delta})=\{0\}$ when $\boldsymbol{\delta} \in \operatorname{int}(C)$. Conversely, when $\boldsymbol{\delta} \in \partial C$, the normal cone $\mathscr{N}_{C}(\boldsymbol{\delta})$ interprets as the cone of outward normals to $C$, as shown on Fig. A.9.right.

Proposition 26 (normal cone).

Let $C \subset \mathbb{R}_{s}^{N \times N}$ be a convex set and any $\boldsymbol{\delta} \in \mathbb{R}_{s}^{N \times N}$. We have:

1. $0 \in \mathscr{N}_{C}(\boldsymbol{\delta}) \Longleftrightarrow \boldsymbol{\delta} \in C$

2. if $C \neq \varnothing$ then $\mathscr{N}_{C}(\boldsymbol{\delta})=\partial \mathscr{I}_{C}(\boldsymbol{\delta})$

3. if $\boldsymbol{\delta} \in \operatorname{int}(C)$ then $\mathscr{N}_{C}(\boldsymbol{\delta})=\{0\}$

Proof: 1. is trivial from the definition of the normal cone.

2. if $C \neq \varnothing$ then $\mathscr{I}_{C}$ is proper. Assume first $\boldsymbol{\delta} \notin C$. Then $\mathscr{N}_{C}(\boldsymbol{\delta})=\partial \mathscr{I}_{C}(\boldsymbol{\delta})=\varnothing$. Next, assume $\boldsymbol{\delta} \in C$. Then

$$
\begin{aligned}
\boldsymbol{\tau} \in \partial \mathscr{I}_{C}(\boldsymbol{\delta}) & \Longleftrightarrow \mathscr{I}_{C}(\boldsymbol{\xi}) \geqslant \mathscr{I}_{C}(\boldsymbol{\delta})+(\boldsymbol{\xi}-\boldsymbol{\delta}): \boldsymbol{\tau}, \quad \forall \boldsymbol{\xi} \in \mathbb{R}_{s}^{N \times N} \\
& \Longleftrightarrow \mathscr{I}_{C}(\boldsymbol{\xi}) \geqslant(\boldsymbol{\xi}-\boldsymbol{\delta}): \boldsymbol{\tau}, \quad \forall \boldsymbol{\xi} \in \mathbb{R}_{s}^{N \times N} \\
& \Longleftrightarrow 0 \geqslant(\boldsymbol{\xi}-\boldsymbol{\delta}): \boldsymbol{\tau}, \quad \forall \boldsymbol{\xi} \in C \\
& \Longleftrightarrow \boldsymbol{\tau} \in \mathscr{N}_{C}(\boldsymbol{\delta})
\end{aligned}
$$

3. Let $\boldsymbol{\delta} \in \operatorname{int}(C)$. There exists $\varepsilon>0$ such that $\mathscr{I}_{C}(\boldsymbol{\delta})$ is zero on the closed ball $B(\boldsymbol{\delta}, \varepsilon)$. Then $\mathscr{I}_{C}(\boldsymbol{\delta})$ is Gâteaux-differentiable at $\boldsymbol{\delta}$ and $\nabla \mathscr{I}_{C}(\boldsymbol{\delta})=0$. Then $\partial \mathscr{I}_{C}(\boldsymbol{\delta})=\{0\}$.

Proposition 27 (normal cone to a convex cone).

Let $K$ be a convex cone. Then, the normal cone $\mathscr{N}_{K}$ admits the following expression, for all $\boldsymbol{\delta} \in K$ :

$$
\mathscr{N}_{K}(\boldsymbol{\delta})= \begin{cases}K^{\circ} \cap\{\boldsymbol{\delta}\}^{\perp} & \text { when } \boldsymbol{\delta} \in K \\ \varnothing & \text { when } \boldsymbol{\delta} \notin K\end{cases}
$$


Proof: The second case holds for any normal cone on any convex set. Let us prove the first one. Let $\boldsymbol{\delta} \in K$ and $\boldsymbol{\tau} \in \mathscr{N}_{K}(\boldsymbol{\delta})$. By definition, we have

$$
(\boldsymbol{\xi}-\boldsymbol{\delta}): \boldsymbol{\tau} \leqslant 0, \quad \boldsymbol{\xi} \in K
$$

Choosing $\boldsymbol{\xi}=0 \in K$ we get $-\boldsymbol{\delta}: \boldsymbol{\tau} \leqslant 0$. Next, choosing $\boldsymbol{\xi}=2 \boldsymbol{\delta} \in K$ we get also $\boldsymbol{\delta}: \boldsymbol{\tau} \leqslant 0$. Then $\boldsymbol{\delta}: \boldsymbol{\tau}=0$ or equivalently $\boldsymbol{\tau} \in\{\boldsymbol{\delta}\}^{\perp}$. Moreover, for all $\boldsymbol{\zeta}=0 \in K$, we have $\boldsymbol{\delta}+\boldsymbol{\zeta}=0 \in K$ and then $\boldsymbol{\zeta}: \boldsymbol{\tau} \leqslant 0$ i.e. $\boldsymbol{\tau} \in K^{\circ}$. Thus, we have $\mathscr{N}_{K}(\boldsymbol{\delta}) \subset K^{\circ} \cap\{\boldsymbol{\delta}\}^{\perp}$. Now, let us prove the reciprocal inclusion. Let $\boldsymbol{\tau} \in K^{\circ} \cap\{\boldsymbol{\delta}\}^{\perp}$ and $\boldsymbol{\xi} \in K$. Then $\boldsymbol{\delta}: \boldsymbol{\tau}=0$ since $\boldsymbol{\tau} \in\{\boldsymbol{\delta}\}^{\perp}$ and $\boldsymbol{\xi}: \boldsymbol{\tau} \leqslant 0$ for any $\boldsymbol{\xi} \in K$ since $\boldsymbol{\tau} \in K^{\circ}$. Then $\boldsymbol{\xi}: \boldsymbol{\tau}-\boldsymbol{\delta}: \boldsymbol{\tau} \leqslant 0$ for any $\boldsymbol{\xi} \in K$. By definition, it means $\boldsymbol{\tau} \in \mathscr{N}_{K}(\boldsymbol{\delta})$ and then $\mathscr{N}_{K}(\boldsymbol{\delta})=K^{\circ} \cap\{\boldsymbol{\delta}\}^{\perp}$.

Proposition 28 (bipolar and bidual of a convex cone).

For any non-empty closed convex cone $K$ we have

$$
K^{* *}=K^{\circ \circ}=K
$$

Proof:

$$
\begin{aligned}
\boldsymbol{\delta} \in K^{\circ \circ} & \Longleftrightarrow \delta \in \mathscr{N}_{K^{\circ}}(0) \text { from proposition } 27 \\
& \Longleftrightarrow \delta \in \partial \mathcal{I}_{K^{\circ}}(0) \text { from proposition 26.c } \\
& \Longleftrightarrow \delta \in \partial\left(\mathcal{I}_{K}\right)^{*}(0) \text { from proposition } 23 \\
& \Longleftrightarrow 0 \in \partial\left(\mathcal{I}_{K}\right)^{* *}(\boldsymbol{\delta}) \text { from theorem } 4 \\
& \Longleftrightarrow 0 \in \partial \mathcal{I}_{K}(\boldsymbol{\delta}) \text { from proposition 19 } \\
& \Longleftrightarrow \delta \in \mathscr{N}_{K}(\boldsymbol{\delta}) \text { from proposition 26.c } \\
& \Longleftrightarrow \delta \text { from proposition 26.b }
\end{aligned}
$$

Proposition 29 (conic complementarity).

For any non-empty closed convex cone $K$ and any $\boldsymbol{\delta}, \boldsymbol{\tau} \in \mathbb{R}^{N \times N}$, we have

$$
K \ni \boldsymbol{\delta} \perp \boldsymbol{\tau} \in K^{\circ} \Longleftrightarrow \boldsymbol{\tau} \in \mathscr{N}_{K}(\boldsymbol{\delta}) \Longleftrightarrow \boldsymbol{\delta} \in \mathscr{N}_{K^{\circ}}(\boldsymbol{\tau})
$$

where $\boldsymbol{\delta} \perp \boldsymbol{\tau}$ denotes $\boldsymbol{\delta}: \boldsymbol{\tau}=0$.

Proof:

$$
\begin{aligned}
\boldsymbol{\tau} \in \mathscr{N}_{K}(\boldsymbol{\delta}) & \Longleftrightarrow \boldsymbol{\tau} \in \partial \mathcal{I}_{K}(\boldsymbol{\delta}) \text { from proposition 26.c } \\
& \Longleftrightarrow \mathcal{I}_{K}(\boldsymbol{\delta})+\mathcal{I}_{K^{*}}(\boldsymbol{\tau})=\boldsymbol{\delta}: \boldsymbol{\tau} \text { from theorem } 4 \\
& \Longleftrightarrow \mathcal{I}_{K}(\boldsymbol{\delta})+\mathcal{I}_{K^{\circ}}(\boldsymbol{\tau})=\boldsymbol{\delta}: \boldsymbol{\tau}
\end{aligned}
$$

The equality is only possible on the effective domain of the left-hand-side, on which $\mathcal{I}_{K}(\boldsymbol{\delta})+\mathcal{I}_{K^{\circ}}(\boldsymbol{\tau})=0$. This means

$$
\mathcal{I}_{K}(\boldsymbol{\delta})+\mathcal{I}_{K^{\circ}}(\boldsymbol{\tau})=\boldsymbol{\delta}: \boldsymbol{\tau} \Longleftrightarrow \boldsymbol{\delta} \in K \text { and } \boldsymbol{\tau} \in K^{\circ} \text { and } \boldsymbol{\delta}: \boldsymbol{\tau}=0
$$

The rightmost equivalence follows from proposition 28 .

Appendix A.5. Von Mises plasticity criterion

Lemma 30 (subdifferential of the matrix norm).

The subdifferential of the function $\varphi: \mathbb{R}_{s}^{N \times N} \rightarrow \mathbb{R}$ is defined for all $\boldsymbol{\delta} \in \mathbb{R}_{s}^{N \times N}$ by

$$
\varphi(\boldsymbol{\delta})=|\boldsymbol{\delta}|
$$

writes

$$
\partial \varphi(\boldsymbol{\delta})= \begin{cases}\left\{\boldsymbol{\tau}=\frac{\boldsymbol{\delta}}{|\boldsymbol{\delta}|}\right\} & \text { when } \boldsymbol{\delta} \neq 0 \\ \left\{\boldsymbol{\tau} \in \mathbb{R}_{s}^{N \times N} /|\boldsymbol{\tau}| \leqslant 1\right\} & \text { otherwise }\end{cases}
$$


Proof: We have $\varphi_{0}(\boldsymbol{\delta})=f \circ g(\boldsymbol{\delta})$ with $f(\xi)=\sqrt{\xi}$, for all $x \in \mathbb{R}$, and $g(\boldsymbol{\delta})=|\boldsymbol{\delta}|^{2}$. Observe that $f^{\prime}(\xi)=1 /(2 \sqrt{\xi})$ and $g^{\prime}(\boldsymbol{\delta})=2 \boldsymbol{\delta}$. Assume first that $\boldsymbol{\delta} \neq 0$. Then, $\varphi$ is differentiable in $\boldsymbol{\delta}$ and

$$
\nabla \varphi(\boldsymbol{\delta})=f^{\prime} \circ g(\boldsymbol{\delta}) g^{\prime}(\boldsymbol{\delta})=\boldsymbol{\delta} /|\boldsymbol{\delta}|
$$

Next, assume $\boldsymbol{\delta}=0$ and let us show that $\partial \varphi(0)=\left\{\boldsymbol{\tau} \in \mathbb{R}_{s}^{N \times N} /|\boldsymbol{\tau}| \leqslant 1\right\}$. The proof of this result is done in two steps. We first assume that $|\boldsymbol{\tau}| \leqslant 1$ and show that then $\boldsymbol{\tau} \in \partial \varphi(0)$. Next, we will show the reciprocal.

step 1: assume $|\boldsymbol{\tau}| \leqslant 1$. By definition of the subdifferential:

$$
\begin{aligned}
\boldsymbol{\tau} \in \partial \varphi(0) & \Longleftrightarrow \varphi(0)+\boldsymbol{\tau}: \boldsymbol{\mu} \leqslant \varphi(\boldsymbol{\mu}), \quad \forall \boldsymbol{\mu} \in \mathbb{R}_{s}^{N \times N} \\
& \Longleftrightarrow \boldsymbol{\tau}: \boldsymbol{\mu} \leqslant|\boldsymbol{\mu}|, \quad \forall \boldsymbol{\mu} \in \mathbb{R}_{s}^{N \times N}
\end{aligned}
$$

From the Cauchy-Schwartz inequality $\boldsymbol{\tau}: \boldsymbol{\mu} \leqslant|\boldsymbol{\tau}||\boldsymbol{\mu}| \leqslant|\boldsymbol{\mu}|$ by assumption, and then $|\boldsymbol{\tau}| \leqslant 1 \Rightarrow \boldsymbol{\tau} \in \partial \varphi(0)$.

step 2: let us turn to the reciprocal. By contraposition:

$$
\begin{aligned}
\boldsymbol{\tau} \in \partial \varphi(0) & \Longrightarrow|\boldsymbol{\tau}| \leqslant 1 \\
& \boldsymbol{\tau} \notin \partial \varphi(0) \Longleftarrow|\boldsymbol{\tau}|>1
\end{aligned}
$$

By definition of the subdifferential:

$$
\begin{aligned}
\boldsymbol{\tau} \in \partial \varphi(0) & \Longleftrightarrow \varphi(0)+\boldsymbol{\tau}:(\boldsymbol{\mu}-0) \leqslant \varphi(\boldsymbol{\mu}), \forall \boldsymbol{\mu} \in \mathbb{R}_{s}^{N \times N} \\
& \Longleftrightarrow \boldsymbol{\tau}: \boldsymbol{\mu} \leqslant|\boldsymbol{\mu}|, \forall \boldsymbol{\mu} \in \mathbb{R}_{s}^{N \times N} \\
\boldsymbol{\tau} \notin \partial \varphi(0) & \Longleftrightarrow \exists \boldsymbol{\mu} \in \mathbb{R}_{s}^{N \times N} / \boldsymbol{\tau}: \boldsymbol{\mu}>|\boldsymbol{\mu}|
\end{aligned}
$$

Assume $|\boldsymbol{\tau}|>1$. We have $\boldsymbol{\tau} \neq 0 \boldsymbol{\mu}=\boldsymbol{\tau} /|\boldsymbol{\tau}|$. Observe that $|\boldsymbol{\mu}|=1$ and then

$$
\boldsymbol{\tau}: \boldsymbol{\mu}-|\boldsymbol{\mu}|=\frac{\boldsymbol{\tau}: \boldsymbol{\tau}}{|\boldsymbol{\tau}|}-1=|\boldsymbol{\tau}|-1>0
$$

Finally $\boldsymbol{\tau} \in \partial \varphi(0) \Longrightarrow|\boldsymbol{\tau}| \leqslant 1$ and the proof is complete.

Definition 31 (von Mises dissipation potential).

The von Mises dissipation potential $\varphi_{m}: \mathbb{R}_{s}^{N \times N} \rightarrow \mathbb{R}$ is defined for all $\boldsymbol{\delta} \in \mathbb{R}_{s}^{N \times N}$ by

$$
\varphi_{m}(\boldsymbol{\delta})=\sigma_{y}|\operatorname{dev} \boldsymbol{\delta}|
$$

where $\sigma_{y} \geqslant 0$ is the yield stress.

Proposition 32 (subdifferential of the von Mises dissipation potential).

Let $\varphi_{m}$ denotes the von Mises viscoplastic dissipation potential, as introduced in definition 31. Its subdifferential expresses, for all $\boldsymbol{\delta} \in \mathbb{R}_{s}^{N \times N}$, as

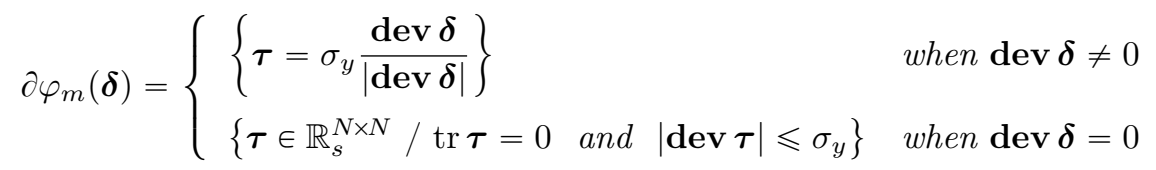

Proof: Observe that the von Mises potential writes also as $\varphi_{m}(\boldsymbol{\delta})=\sigma_{y} \varphi(\operatorname{dev} \boldsymbol{\delta})$ where $\varphi(\boldsymbol{\delta})=|\boldsymbol{\delta}|$ is the matrix norm. Then, applying proposition 16 and lemma 30 , we get

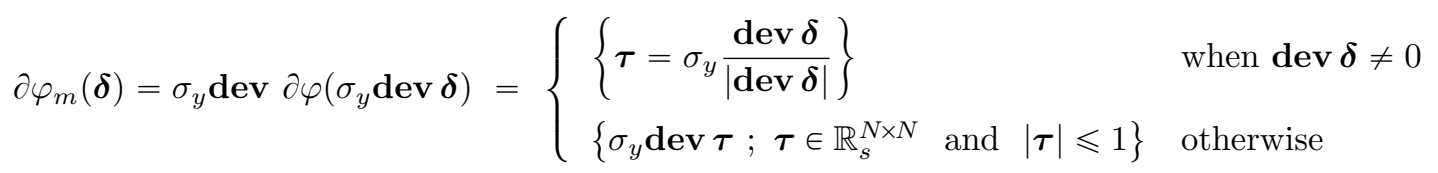

which leads to (A.3b) after rearrangements. 
Definition 33 (von Mises set).

For all $\sigma_{y} \geqslant 0$, the von Mises set is defined by

$$
B_{\sigma_{y}}=\left\{\boldsymbol{\tau} \in \mathbb{R}_{s}^{N \times N} ;|\operatorname{dev} \boldsymbol{\tau}| \leqslant \sigma_{y}\right\}
$$

Note that the von Mises set is a convex cone, that corresponds to a vertical band of width $\sigma_{y}$ in the deviatoric-trace stress plane representation.

Proposition 34 (von Mises set).

For all $\sigma_{y} \geqslant 0$, we have

$$
\left(\mathcal{I}_{-B_{\sigma_{y}}}\right)^{*}=\mathcal{I}_{\text {ker(tr) }}+\varphi_{m}
$$

Proof: From corollary 15, the equality could be shown from the equality of the subdifferentials. From definition 24 and proposition 26, a necessary condition for $\partial \mathscr{I}_{\operatorname{ker}(\operatorname{tr})}(\boldsymbol{\delta})$ to be non-empty is $\boldsymbol{\delta} \in \operatorname{ker}(\operatorname{tr})$ i.e. $\operatorname{tr} \boldsymbol{\delta}=0$. Next, proposition 17, gives $\partial \mathscr{I}_{\operatorname{ker}(\operatorname{tr})}(\boldsymbol{\delta})=\operatorname{ker}(\mathbf{d e v})$.

- forward inclusion: $\partial\left(\mathscr{I}_{-B_{\sigma_{y}}}\right)^{*} \subset \operatorname{ker}(\mathbf{d e v})+\partial \varphi_{m}$

Let $\boldsymbol{\tau} \in \partial\left(\mathscr{I}_{-B_{\sigma_{y}}}\right)^{*}(\boldsymbol{\delta})$ or equivalently $\boldsymbol{\delta} \in \partial \mathscr{I}_{-B_{\sigma_{y}}}(\boldsymbol{\tau})=\mathscr{N}_{-B_{\sigma_{y}}}(\boldsymbol{\tau})$ where we have used the Fenchel-Young theorem 4 and proposition 26. From definition 24, for $\mathscr{N}_{-B_{\sigma_{y}}}(\boldsymbol{\tau})$ to be non-empty, we necessarily have $\boldsymbol{\tau} \in-B_{\sigma_{y}}$ i.e., by definition $40,|\boldsymbol{d e v} \boldsymbol{\tau}| \leqslant \sigma_{y}$. Expanding definition 24 of a normal cone, we have

$$
\begin{aligned}
& \operatorname{tr} \boldsymbol{\delta}=0 \\
& |\operatorname{dev} \boldsymbol{\tau}| \leqslant \sigma_{y} \\
& (\boldsymbol{\xi}-\boldsymbol{\tau}): \boldsymbol{\delta} \leqslant 0, \quad \forall \boldsymbol{\xi} /|\mathbf{d e v} \boldsymbol{\xi}| \leqslant \sigma_{y}
\end{aligned}
$$

First, observe that $\frac{\operatorname{tr} \boldsymbol{\tau}}{N} \boldsymbol{I} \in \operatorname{ker}(\mathbf{d e v})$. Assume first that $\operatorname{dev} \boldsymbol{\delta}=0$, which means from (A.4) that $\boldsymbol{\delta}=0$, then, from proposition 32, we obtain $\operatorname{dev} \boldsymbol{\tau} \in \partial \varphi_{m}(\boldsymbol{\delta})$. Next, assume $\operatorname{dev} \boldsymbol{\delta} \neq 0$. Suppose, by reductio ad absurdum that $\boldsymbol{\tau} \notin \partial \varphi_{m}(\boldsymbol{\delta})$. Since we necessarily have $|\boldsymbol{\tau}|=\sigma_{y}$ for (A.6) to be satisfied, there exists $\boldsymbol{\delta}_{2}$ such that $\operatorname{dev} \boldsymbol{\tau}=\sigma_{y} \frac{\operatorname{dev} \boldsymbol{\delta}_{2}}{\left|\operatorname{dev} \boldsymbol{\delta}_{2}\right|}$ and $\left(\operatorname{dev} \boldsymbol{\delta}_{2}\right):(\operatorname{dev} \boldsymbol{\delta})<\left|\operatorname{dev} \boldsymbol{\delta}_{2}\right||\operatorname{dev} \boldsymbol{\delta}|$ i.e. $\operatorname{dev} \boldsymbol{\delta}$ and $\operatorname{dev} \boldsymbol{\delta}_{2}$ are not aligned. Then, choosing $\operatorname{dev} \boldsymbol{\xi}=\sigma_{y} \frac{\operatorname{dev} \boldsymbol{\delta}}{|\operatorname{dev} \boldsymbol{\delta}|}$ in (A.6) leads to $\left(\operatorname{dev} \boldsymbol{\delta}_{2}\right):(\operatorname{dev} \boldsymbol{\delta}) \geqslant\left|\operatorname{dev} \boldsymbol{\delta}_{2}\right||\operatorname{dev} \boldsymbol{\delta}|$ which is impossible. Then $\boldsymbol{\tau} \in \partial \varphi_{m}(\boldsymbol{\delta})$ and the forward inclusion is complete.

- backward inclusion: $\partial\left(\mathscr{I}_{-B_{\sigma_{y}}}\right)^{*} \supset \operatorname{ker}(\mathbf{d e v})+\partial \varphi_{m}$

Let any $\boldsymbol{\tau} \in \operatorname{ker}(\mathbf{d e v})+\partial \varphi_{m}(\boldsymbol{\delta})$ with $\frac{\operatorname{tr} \boldsymbol{\tau}}{N} \boldsymbol{I} \in \operatorname{ker}(\mathbf{d e v})$ and $\operatorname{dev} \boldsymbol{\tau} \in \partial \varphi_{m}(\boldsymbol{\delta})$. Then, from proposition 32, we have $|\operatorname{dev} \boldsymbol{\tau}| \leqslant \sigma_{y}$ and thus, by definition 40 we obtain $\boldsymbol{\tau} \in B_{\sigma_{y}}$. From definition 24, note also that $\mathscr{N}_{-B_{\sigma_{y}}}(\boldsymbol{\tau}) \neq \varnothing$. Assume first that $\operatorname{dev} \boldsymbol{\delta} \neq 0$. Then, from proposition 32 , and since $\boldsymbol{\tau} \in \partial \varphi_{m}(\boldsymbol{\delta})$ we get $\operatorname{dev} \boldsymbol{\tau}=\sigma_{y} \frac{\operatorname{dev} \boldsymbol{\delta}}{|\operatorname{dev} \boldsymbol{\delta}|}$. Note that $\boldsymbol{\tau}: \boldsymbol{\delta}=\sigma_{y}|\operatorname{dev} \boldsymbol{\delta}|$. For all $\boldsymbol{\xi}$ such that $|\operatorname{dev} \boldsymbol{\xi}| \leqslant \sigma_{y}$ we have

$$
\begin{aligned}
& \xi: \delta=(\operatorname{dev} \xi):(\operatorname{dev} \delta) \leqslant|\operatorname{dev} \xi||\operatorname{dev} \delta| \leqslant \sigma_{y}|\operatorname{dev} \delta|=\tau: \delta \\
\Longleftrightarrow & (\xi-\tau): \delta \leqslant 0
\end{aligned}
$$

Assume next that $\operatorname{dev} \boldsymbol{\delta}=0$. Since we also have $\operatorname{tr} \boldsymbol{\delta}=0$ we get $\boldsymbol{\delta}=0$ and (A.7) is also trivially satisfied. It means that $\boldsymbol{\delta} \in \mathscr{N}_{-B_{\sigma_{y}}}(\boldsymbol{\tau})$ or equivalently $\boldsymbol{\tau} \in \partial\left(\mathscr{I}_{-B_{\sigma_{y}}}\right)^{*}(\boldsymbol{\delta})$ where we have used the Fenchel-Young theorem 4 and proposition 26. Then the proof of the subdifferentials equality is complete. 
Appendix A.6. Drucker-Prager plasticity criterion

Definition 35 (Drucker-Prager cone).

For all $\mu \in[0, \infty]$, the Drucker-Prager cone, denoted by $K_{\mu}$ is the second-order cone defined by:

$$
K_{\mu}= \begin{cases}\operatorname{ker}(\mathbf{d e v}) & \text { when } \mu=0 \\ \left\{\boldsymbol{\tau} \in \mathbb{R}_{s}^{N \times N} ;|\operatorname{dev} \boldsymbol{\tau}| \leqslant \frac{\mu \operatorname{tr} \boldsymbol{\tau}}{\sqrt{N}}\right\} & \text { when } \mu \in] 0, \infty[ \\ \operatorname{ker}(\operatorname{tr}) & \text { when } \mu=\infty\end{cases}
$$

Proposition 36 (Drucker-Prager cone is convex).

For all $\mu \in[0, \infty]$, the Drucker-Prager cone $K_{\mu}$ is a convex cone.

Proof: Consider any $\boldsymbol{\tau}_{1}, \boldsymbol{\tau}_{2} \in K_{\mu}$ and $\beta_{1}, \beta_{2} \geqslant 0$. Assume first $\mu \in[0, \infty[$. We have:

$$
\begin{aligned}
\left|\operatorname{dev}\left(\beta_{1} \boldsymbol{\tau}_{1}+\beta_{2} \boldsymbol{\tau}_{2}\right)\right| & \leqslant \beta_{1}\left|\operatorname{dev} \boldsymbol{\tau}_{1}\right|+\beta_{2}\left|\operatorname{dev} \boldsymbol{\tau}_{2}\right| \\
& \leqslant \frac{\beta_{1} \mu \operatorname{tr} \boldsymbol{\tau}_{1}}{\sqrt{N}}+\frac{\beta_{2} \mu \operatorname{tr} \boldsymbol{\tau}_{2}}{\sqrt{N}} \\
& =\frac{\mu \operatorname{tr}\left(\beta_{1} \boldsymbol{\tau}_{1}+\beta_{2} \boldsymbol{\tau}_{2}\right)}{\sqrt{N}}
\end{aligned}
$$

and then $\beta_{1} \boldsymbol{\tau}_{1}+\beta_{2} \boldsymbol{\tau}_{2} \in K_{\mu}$. Next, assume $\mu=\infty$. We have:

$$
\operatorname{tr}\left(\beta_{1} \boldsymbol{\tau}_{1}+\beta_{2} \boldsymbol{\tau}_{2}\right)=\beta_{1} \operatorname{tr} \boldsymbol{\tau}_{1}+\beta_{2} \operatorname{tr} \boldsymbol{\tau}_{2}=0
$$

and then $\beta_{1} \boldsymbol{\tau}_{1}+\beta_{2} \boldsymbol{\tau}_{2} \in K_{\infty}$. Then, from definition $20, K_{\mu}$ is a convex cone for all $\mu \in[0, \infty]$.

Proposition 37 (polar and dual Drucker-Prager cones).

For all $\mu \in[0, \infty]$, the polar and dual of the Drucker-Prager cone $K_{\mu}$ are respectively:

$$
\begin{aligned}
& K_{\mu}^{\circ}=-K_{\frac{1}{\mu}} \\
& K_{\mu}^{*}=K_{\frac{1}{\mu}}
\end{aligned}
$$

Proof: When $\mu=0$, from proposition 17 we have $(\operatorname{ker} \mathbf{d e v})^{\circ}=\operatorname{ker}(\operatorname{tr})$ and from definition 35 we deduce immediately $K_{0}^{\circ}=-K_{\infty}$. Tacking the polar of the previous relation and using proposition 28, we get $K_{\infty}^{\circ}=-K_{0}^{\circ}=-K_{0}$ which completes the proof for $\mu=\infty$. The rest of the proof is devoted to the case $\mu \in] 0, \infty[$.

- forward inclusion $K_{\mu}^{\circ} \subset-K_{\frac{1}{\mu}}$.

Let $\boldsymbol{\delta} \in K_{\mu}^{\circ}$ and let us prove that $\boldsymbol{\delta} \in-K_{\frac{1}{\mu}}$. If $\boldsymbol{\delta}=0$ then $\boldsymbol{\delta} \in-K_{\frac{1}{\mu}}$. Next, assume $\boldsymbol{\delta} \neq 0$. By definition 22, for all $\boldsymbol{\tau} \in K_{\mu}$, the inequality $\delta: \tau \leqslant 0$ holds. Then, let us choose:

$$
\boldsymbol{\tau}=\frac{\operatorname{dev} \boldsymbol{\delta}}{|\operatorname{dev} \boldsymbol{\delta}|}+\frac{1}{\sqrt{N} \mu} \boldsymbol{I}
$$

such that $|\operatorname{dev} \boldsymbol{\tau}|=\frac{\mu \operatorname{tr} \boldsymbol{\tau}}{\sqrt{N}}$ and then $\boldsymbol{\tau} \in K_{\mu}$. With this choice, we get:

$$
\begin{aligned}
\delta: \tau \leqslant 0 & \Longleftrightarrow \frac{(\operatorname{tr} \boldsymbol{\delta})(\operatorname{tr} \boldsymbol{\tau})}{N}+(\operatorname{dev} \boldsymbol{\delta}):(\operatorname{dev} \boldsymbol{\tau}) \leqslant 0 \text { by expansion } \\
& \Longleftrightarrow|\operatorname{dev} \boldsymbol{\delta}| \leqslant-\frac{\operatorname{tr} \boldsymbol{\delta}}{\sqrt{N} \mu} \\
& \Longleftrightarrow \delta \in-K_{\frac{1}{\mu}}
\end{aligned}
$$


- backward inclusion $K_{\mu}^{\circ} \supset-K_{\frac{1}{\mu}}$.

Conversely, let $\boldsymbol{\delta} \in-K_{\frac{1}{\mu}}$ and let us prove that $\boldsymbol{\delta} \in K_{\mu}^{\circ}$. For all $\boldsymbol{\tau} \in K_{\mu}$ we have:

$$
\begin{aligned}
& 0 \leqslant|\operatorname{dev} \boldsymbol{\delta}| \leqslant-\frac{\operatorname{tr} \boldsymbol{\delta}}{\sqrt{N} \mu} \\
& 0 \leqslant|\operatorname{dev} \boldsymbol{\tau}| \leqslant \frac{\mu \operatorname{tr} \boldsymbol{\tau}}{\sqrt{N}}
\end{aligned}
$$

Then

$$
\begin{aligned}
\boldsymbol{\delta}: \boldsymbol{\tau} & =(\operatorname{dev} \boldsymbol{\delta}):(\operatorname{dev} \boldsymbol{\tau})+\frac{(\operatorname{tr} \boldsymbol{\delta})(\operatorname{tr} \boldsymbol{\tau})}{N} \text { by expansion in deviatoric and spherical parts } \\
& \leqslant|\operatorname{dev} \boldsymbol{\delta}||\operatorname{dev} \boldsymbol{\tau}|+\frac{(\operatorname{tr} \boldsymbol{\delta})(\operatorname{tr} \boldsymbol{\tau})}{N} \text { from the Cauchy-Schwartz inequality } \\
& \leqslant-\frac{\operatorname{tr} \boldsymbol{\delta}}{\sqrt{N} \mu} \times \frac{\mu \operatorname{tr} \boldsymbol{\tau}}{\sqrt{N}}+\frac{(\operatorname{tr} \boldsymbol{\delta})(\operatorname{tr} \boldsymbol{\tau})}{N} \text { since } \boldsymbol{\delta} \in-K_{\frac{1}{\mu}} \text { and } \boldsymbol{\tau} \in K_{\mu} \\
& =0
\end{aligned}
$$

By definition 22, it means that $\delta \in K_{\mu}^{\circ}$. Thus, we have $K_{\mu}^{\circ}=-K_{\frac{1}{\mu}}$ for all $\mu \in[0, \infty]$. Finally, from definition $22,\left(K_{\mu}\right)^{*}=-\left(K_{\mu}\right)^{\circ}=K_{\frac{1}{\mu}}$ and the proof is complete.

Corollary 38 (conjugate of the indicator to the Drucker-Prager cone).

For all $\mu \in[0, \infty]$, we have

$$
\left(\mathscr{I}_{-K_{\mu}}\right)^{*}=\mathscr{I}_{K_{\frac{1}{\mu}}}
$$

As a consequence, for all $\boldsymbol{\delta}, \boldsymbol{\tau} \in \mathbb{R}_{s}^{N \times N}$,

$$
\begin{aligned}
-\boldsymbol{\tau} \in \partial \mathscr{I}_{K_{\frac{1}{\mu}}}(\boldsymbol{\delta}) & \Longleftrightarrow-\boldsymbol{\delta} \in \partial \mathscr{I}_{K_{\mu}}(\boldsymbol{\tau}) \\
& \Longleftrightarrow K_{\frac{1}{\mu}} \ni \boldsymbol{\delta} \text { and } \boldsymbol{\delta}: \boldsymbol{\tau}=0 \text { and } \boldsymbol{\tau} \in K_{\mu}
\end{aligned}
$$

Proof: From corollary 15, the equality could be shown from the equality of the differentials. The equality of the differentials $\partial\left(\mathscr{I}_{-K_{\mu}}\right)^{*}=\partial \mathscr{I}_{K_{\frac{1}{\mu}}}$ is obtained by using the Fenchel-Young theorem 4 , and then successively propositions $23,37^{\mu}$ and 26 . The consequence is then deduced from proposition 29 .

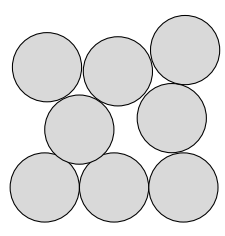

sticking

$\boldsymbol{\delta}=0$

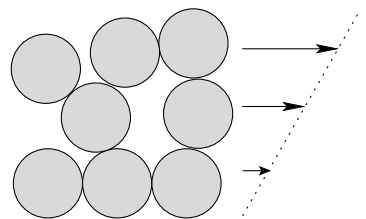

sliding

$\boldsymbol{\delta} \neq 0, \boldsymbol{\tau} \neq 0$

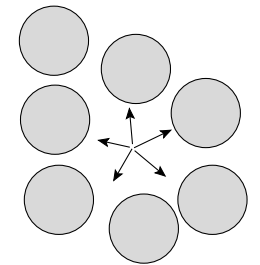

loosing contacts $\boldsymbol{\tau}=0$

Figure A.10: Disjunctive formulation of the Drucker-Prager plasticity condition $\boldsymbol{\tau} \in \partial_{K_{\frac{1}{\mu}}}(\boldsymbol{\delta})$.

Proposition 39 (disjunctive formulation).

For all $\mu \in] 0, \infty\left[\right.$, and all $\boldsymbol{\delta}, \boldsymbol{\tau} \in \mathbb{R}_{s}^{N \times N}$, we have $\boldsymbol{\tau} \in \partial \mathscr{I}_{K_{\frac{1}{\mu}}}(\boldsymbol{\delta})$, or equivalently $-\boldsymbol{\delta} \in \partial \mathscr{I}_{K_{\mu}}(-\boldsymbol{\tau})$, 
if and only if one of the three condition is satisfied (see Fig. A.10):

$$
\begin{aligned}
& \text { (i) sticking: } \quad|\operatorname{dev} \boldsymbol{\tau}| \leqslant-\frac{\mu \operatorname{tr} \boldsymbol{\tau}}{\sqrt{N}} \text { and } \delta=0 \\
& \text { (ii) sliding: } \left.\quad \begin{array}{rl}
\operatorname{dev} \boldsymbol{\tau} & =-\frac{\mu \operatorname{tr} \boldsymbol{\tau}}{\sqrt{N}} \frac{\operatorname{dev} \boldsymbol{\delta}}{|\operatorname{dev} \boldsymbol{\delta}|} \\
\operatorname{tr} \boldsymbol{\tau} & \leqslant 0
\end{array}\right\} \text { and }|\operatorname{dev} \boldsymbol{\delta}|=\frac{\operatorname{tr} \boldsymbol{\delta}}{\sqrt{N \mu}} \neq 0 \\
& \text { (iii) losing contact: } \quad \boldsymbol{\tau}=0 \text { and }|\operatorname{dev} \boldsymbol{\delta}|<\frac{\operatorname{tr} \boldsymbol{\delta}}{\sqrt{N} \mu}
\end{aligned}
$$

When $\mu=0: \quad \boldsymbol{\tau} \in \partial \mathscr{I}_{K_{\infty}}(\boldsymbol{\delta}) \Longleftrightarrow \operatorname{tr} \boldsymbol{\delta}=0$ and $\operatorname{dev} \boldsymbol{\tau}=0$.

When $\mu=\infty: \boldsymbol{\tau} \in \partial \mathscr{I}_{K_{0}}(\boldsymbol{\delta}) \Longleftrightarrow \operatorname{dev} \boldsymbol{\delta}=0$ and $\operatorname{tr} \boldsymbol{\tau}=0$.

Proof: The case $\mu \in\{0, \infty\}$ is a direct consequence of definition 35 and proposition 38, so let assume $\mu \in] 0, \infty\left[\right.$. From proposition $38, \boldsymbol{\tau} \in \partial \mathscr{I}_{K_{\frac{1}{\mu}}}(\boldsymbol{\delta})$ if and only if $K_{\frac{1}{\mu}} \ni \boldsymbol{\delta}$ and $\boldsymbol{\delta}: \boldsymbol{\tau}=0$ and $-\boldsymbol{\tau} \in K_{\mu}$ i.e.

$$
\left\{\begin{array}{l}
|\operatorname{dev} \boldsymbol{\delta}| \leqslant \frac{\operatorname{tr} \boldsymbol{\delta}}{\sqrt{N} \mu} \\
(\operatorname{dev} \boldsymbol{\delta}):(\operatorname{dev} \boldsymbol{\tau})+\frac{(\operatorname{tr} \boldsymbol{\delta})(\operatorname{tr} \boldsymbol{\tau})}{N}=0 \\
|\operatorname{dev} \boldsymbol{\tau}| \leqslant-\frac{\mu \operatorname{tr} \boldsymbol{\tau}}{\sqrt{N}}
\end{array}\right.
$$

It is easy to check, from the definition of the Drucker-Prager cone, that any of the three case of the disjunctive formulation is a sufficient condition for $\boldsymbol{\tau} \in \partial \mathscr{I}_{K_{\frac{1}{\mu}}}(\boldsymbol{\delta})$. So, let us turn to show that it is a necessary condition.

- Let us first assume $\boldsymbol{\delta}=0$. Then (A.9c) directly gives the sticking case (A.8a).

- Next, assume $\boldsymbol{\delta} \neq 0$ and $\operatorname{tr} \boldsymbol{\delta}=\sqrt{N} \mu|\operatorname{dev} \boldsymbol{\delta}|$. Then $\operatorname{dev} \boldsymbol{\delta} \neq 0$. From (A.9b) and (A.9c), we have:

$$
\begin{aligned}
(\operatorname{dev} \boldsymbol{\delta}):(\operatorname{dev} \boldsymbol{\tau}) & =-\frac{(\operatorname{tr} \boldsymbol{\delta})(\operatorname{tr} \boldsymbol{\tau})}{N} \text { from (A.9b) } \\
& \geqslant|\operatorname{dev} \boldsymbol{\delta}||\operatorname{dev} \boldsymbol{\tau}| \text { from (A.9a) and (A.9c) }
\end{aligned}
$$

and from the Cauchy-Schwartz inequality, the equality occurs. Thus, there exists a constant $k \in \mathbb{R}$ such that $\operatorname{dev} \boldsymbol{\tau}=k \operatorname{dev} \boldsymbol{\delta}$. From (A.9b) and since $\operatorname{dev} \boldsymbol{\delta} \neq 0$, we obtain an expression for $k$

$$
k=-\frac{(\operatorname{tr} \boldsymbol{\delta})(\operatorname{tr} \boldsymbol{\tau})}{N|\operatorname{dev} \boldsymbol{\delta}|^{2}}=-\frac{\sqrt{N} \mu \operatorname{tr} \boldsymbol{\tau}}{N|\operatorname{dev} \boldsymbol{\delta}|} \text { since } \operatorname{tr} \boldsymbol{\delta}=\sqrt{N} \mu|\operatorname{dev} \boldsymbol{\delta}|
$$

Note that $k \geqslant 0$ since $\operatorname{tr} \tau \leqslant 0$ from (A.9c) and finally, the sliding case (A.8b) is complete.

- Finally, assume $\boldsymbol{\delta} \neq 0$ and $\operatorname{tr} \boldsymbol{\delta} \neq \sqrt{N} \mu|\operatorname{dev} \boldsymbol{\delta}|$. From (A.9a), we then have $\operatorname{tr} \boldsymbol{\delta}>\sqrt{N} \mu|\operatorname{dev} \boldsymbol{\delta}|$ and thus $\operatorname{tr} \boldsymbol{\delta} \neq 0$. From (A.9c), we obtain $\operatorname{tr} \boldsymbol{\tau} \leqslant 0$ and suppose, by reductio ad absurdum that $\operatorname{tr} \tau<0$. Then, from the Cauchy-Schwartz inequality:

$$
(\operatorname{dev} \boldsymbol{\delta}):(\operatorname{dev} \boldsymbol{\tau}) \leqslant|\operatorname{dev} \boldsymbol{\delta}||\operatorname{dev} \boldsymbol{\tau}|<-\frac{\operatorname{tr} \boldsymbol{\delta}}{\sqrt{N} \mu} \frac{\mu \operatorname{tr} \boldsymbol{\tau}}{\sqrt{N}}=-\frac{(\operatorname{tr} \boldsymbol{\delta})(\operatorname{tr} \boldsymbol{\tau})}{N}
$$

This implies $\boldsymbol{\delta}: \boldsymbol{\tau}<0$ which is in contradiction with (A.9b). Then $\operatorname{tr} \boldsymbol{\tau}=0$. Again from (A.9c) we obtain $\operatorname{dev} \boldsymbol{\tau}=0$ and finally $\boldsymbol{\tau}=0$. Then, the losing contact case (A.8c) is complete. 


\section{Appendix A.7. Translated Drucker-Prager plasticity criterion}

This criterion extends the original Drucker-Prager one [14] by incorporating the cohesion $\sigma_{y} \geqslant 0$ (see e.g. [1]).

Definition 40 (translated Drucker-Prager cone). For all $\mu \in[0, \infty]$ and $\sigma_{y} \geqslant 0$, the translated Drucker-Prager cone, denoted by $T_{\mu, \sigma_{y}}$, is defined by:

$$
T_{\mu, \sigma_{y}}= \begin{cases}\left\{\boldsymbol{\tau} \in \mathbb{R}_{s}^{N \times N} ;|\operatorname{dev} \boldsymbol{\tau}| \leqslant \sigma_{y}\right\}=B_{\sigma_{y}} & \text { when } \mu=0 \\ \left\{\boldsymbol{\tau} \in \mathbb{R}_{s}^{N \times N} ;|\operatorname{dev} \boldsymbol{\tau}| \leqslant \sigma_{y}+\frac{\mu \operatorname{tr} \boldsymbol{\tau}}{\sqrt{N}}\right\} & \text { when } \mu \in] 0, \infty[ \\ \left\{\boldsymbol{\tau} \in \mathbb{R}_{s}^{N \times N} ; \operatorname{tr} \boldsymbol{\tau}=0\right\}=\operatorname{ker}(\operatorname{tr}) & \text { when } \mu=\infty\end{cases}
$$

Proposition 41 (translated Drucker-Prager cone).

For all $\mu \in[0, \infty]$ and $\sigma_{y} \geqslant 0$, the translated Drucker-Prager cone $T_{\mu, \sigma_{y}}$ is a convex set and the dual of its indicator function writes: for all $\boldsymbol{\delta} \in \mathbb{R}_{s}^{N \times N}$ :

$$
\left(\mathscr{I}_{-T_{\mu, \sigma_{y}}}\right)^{*}(\boldsymbol{\delta})= \begin{cases}\mathscr{I}_{\operatorname{ker}(\operatorname{tr})}(\boldsymbol{\delta})+\varphi_{m}(\boldsymbol{\delta}) & \text { when } \mu=0 \\ \mathscr{I}_{K_{\frac{1}{\mu}}}(\boldsymbol{\delta})+\frac{\sigma_{y}}{\sqrt{N} \mu} \operatorname{tr} \boldsymbol{\delta} & \text { when } \mu \in] 0, \infty[ \\ \mathscr{I}_{\operatorname{ker}(\mathbf{d e v})}(\boldsymbol{\delta}) & \text { when } \mu=\infty\end{cases}
$$

Proof: Note that when $\sigma_{y}=0$, we have $T_{\mu, \sigma_{y}}=K_{\mu}$ which is a convex set and the result is given by proposition 38 . Thus, the present result extends proposition 38 .

Let us turn now to the general case $\sigma_{y} \geqslant 0$. The convexity of $T_{\mu, \sigma_{y}}$ is established by using an argument similar to those of the proof of proposition 36. For establishing the main result, we then successively consider the three cases $\mu=0, \mu=\infty$ and $\mu \in] 0, \infty\left[\right.$. When $\mu=0$, since $T_{0, \sigma_{y}}=$ $B_{\sigma_{y}}$, the result is directly obtained from proposition 34 . When $\mu=\infty$, from definition 35 , we have $-T_{\infty, \sigma_{y}}=\operatorname{ker}(\operatorname{tr})$ and then, from proposition 17 we get $\left(\mathscr{I}_{-T_{\infty, \sigma_{y}}}\right)^{*}=\mathscr{I}_{\operatorname{ker}(\operatorname{tr})}^{*}=\mathscr{I}_{\operatorname{ker}(\mathbf{d e v})}$ and then, the proof is also complete when $\mu=\infty$. The rest of the proof is dedicated to the case $\mu \in] 0, \infty[$.

From corollary 15, the equality could be shown from the equality of the differentials. Assume first that $\delta \notin K_{\frac{1}{\mu}}$ then both $\partial \mathscr{I}_{K_{1}}$ and $\partial\left(\mathscr{I}_{-T_{\mu, \sigma_{y}}}\right)^{*}$ are empty and the equality is satisfied. Then, assume $\boldsymbol{\delta} \in K_{\frac{1}{\mu}}$ such that $\partial \mathscr{I}_{K_{\frac{1}{\mu}}}^{{ }^{\bar{\mu}}}(\boldsymbol{\delta}) \neq \varnothing$. For the equality, we successively prove the forward and reverse inclusions of the subdifferentials.

- forward inclusion: $\partial\left(\mathscr{I}_{-T_{\mu, \sigma_{y}}}\right)^{*} \subset \partial \mathscr{I}_{K_{\frac{1}{\mu}}}+\frac{\sigma_{y}}{\sqrt{N} \mu} \boldsymbol{I}$

Let $\boldsymbol{\tau} \in \partial\left(\mathscr{I}_{-T_{\mu, \sigma_{y}}}\right)^{*}(\boldsymbol{\delta})$ or equivalently $\boldsymbol{\delta} \in \partial \mathscr{I}_{-T_{\mu, \sigma_{y}}}(\boldsymbol{\tau})=\mathscr{N}_{-T_{\mu, \sigma_{y}}}(\boldsymbol{\tau})$ where we have used the Fenchel-Young theorem 4 and proposition 26. From definition 24, we necessarily have $\boldsymbol{\tau} \in-T_{\mu, \sigma_{y}}$ for $\mathscr{N}_{-T_{\mu, \sigma_{y}}}(\boldsymbol{\tau})$ to be non-empty. Expanding $\boldsymbol{\delta} \in K_{\frac{1}{\mu}}, \boldsymbol{\tau} \in-T_{\mu, \sigma_{y}}$ and definition 24 of a normal cone, we have

$$
\left\{\begin{array}{l}
|\operatorname{dev} \boldsymbol{\delta}| \leqslant \frac{\operatorname{tr} \boldsymbol{\delta}}{\sqrt{N} \mu} \\
|\operatorname{dev} \boldsymbol{\tau}| \leqslant-\frac{\mu \operatorname{tr} \boldsymbol{\tau}}{\sqrt{N}}+\sigma_{y} \\
(\boldsymbol{\xi}-\boldsymbol{\tau}): \boldsymbol{\delta} \leqslant 0, \quad \forall \boldsymbol{\xi} /|\operatorname{dev} \boldsymbol{\xi}| \leqslant-\frac{\mu \operatorname{tr} \boldsymbol{\xi}}{\sqrt{N}}+\sigma_{y}
\end{array}\right.
$$


By definition 40 of $T_{\mu, \sigma_{y}}$, note that $\boldsymbol{\tau} \in-T_{\mu, \sigma_{y}}$ is equivalent to $\boldsymbol{\tau}-\frac{\sigma_{y}}{\sqrt{N} \mu} \boldsymbol{I} \in-K_{\mu}$. Next, let us expand

$$
\begin{aligned}
\left(\boldsymbol{\tau}-\frac{\sigma_{y}}{\sqrt{N} \mu} \boldsymbol{I}\right): \boldsymbol{\delta}= & (\operatorname{dev} \boldsymbol{\tau}):(\operatorname{dev} \boldsymbol{\delta})+\frac{(\operatorname{tr} \boldsymbol{\tau})(\operatorname{tr} \boldsymbol{\delta})}{N}-\frac{\sigma_{y}}{\sqrt{N} \mu} \operatorname{tr} \boldsymbol{\delta} \\
& \leqslant|\operatorname{dev} \boldsymbol{\tau}||\mathbf{d e v} \boldsymbol{\delta}|+\frac{(\operatorname{tr} \boldsymbol{\tau})(\operatorname{tr} \boldsymbol{\delta})}{N}-\frac{\sigma_{y}}{\sqrt{N} \mu} \operatorname{tr} \boldsymbol{\delta} \\
& \text { from the Cauchy-Schwartz inequality } \\
& \leqslant\left(-\frac{\mu \operatorname{tr} \boldsymbol{\tau}}{\sqrt{N}}+\sigma_{y}\right) \frac{\operatorname{tr} \boldsymbol{\delta}}{\sqrt{N} \mu}+\frac{(\operatorname{tr} \boldsymbol{\tau})(\operatorname{tr} \boldsymbol{\delta})}{N}-\frac{\sigma_{y}}{\sqrt{N} \mu} \operatorname{tr} \boldsymbol{\delta} \\
& \text { from (A.11) and (A.12) } \\
& =0
\end{aligned}
$$

Conversely, choosing $\boldsymbol{\xi}=\frac{\sigma_{y}}{\sqrt{N} \mu} \boldsymbol{I} \in-T_{\mu, \sigma_{y}}$ in (A.13) leads to $\left(\boldsymbol{\tau}-\frac{\sigma_{y}}{\sqrt{N} \mu} \boldsymbol{I}\right): \boldsymbol{\delta} \leqslant 0$ and then $\left(\boldsymbol{\tau}-\frac{\sigma_{y}}{\sqrt{N} \mu} \boldsymbol{I}\right): \boldsymbol{\delta}=0$. Then $\boldsymbol{\tau}-\frac{\sigma_{y}}{\sqrt{N} \mu} \boldsymbol{I} \in-K_{\mu} \cap\{\boldsymbol{\delta}\}^{\perp}=\left(K_{\frac{1}{\mu}}\right)^{\circ} \cap\{\boldsymbol{\delta}\}^{\perp}=\partial \mathscr{I}_{K_{\frac{1}{\mu}}}(\boldsymbol{\delta})$ where we have used propositions 26, 27 and 37. This means that $\boldsymbol{\tau} \in \partial \mathscr{I}_{K_{\frac{1}{\mu}}}(\boldsymbol{\delta})+\frac{\sigma_{y}}{\sqrt{N} \mu} \boldsymbol{I}$ and the forward inclusion is complete.

- backward inclusion: $\partial\left(\mathscr{I}_{-T_{\mu, \sigma_{y}}}\right)^{*} \supset \partial_{K_{\frac{1}{\mu}}}+\frac{\sigma_{y}}{\sqrt{N} \mu} \boldsymbol{I}$

Let any $\boldsymbol{\tau} \in \partial \mathscr{I}_{K_{\frac{1}{\mu}}}(\boldsymbol{\delta})+\frac{\sigma_{y}}{\sqrt{N} \mu} \boldsymbol{I}$. We have

$$
\left\{\begin{array}{l}
\boldsymbol{\delta} \in K_{\frac{1}{\mu}} \\
\boldsymbol{\tau}-\frac{\sigma_{y}}{\sqrt{N} \mu} \boldsymbol{I} \in \partial \mathscr{I}_{K_{\frac{1}{\mu}}}(\boldsymbol{\delta})=\mathscr{N}_{K_{\frac{1}{\mu}}}(\boldsymbol{\delta})=\left(K_{\frac{1}{\mu}}\right)^{\circ} \cap\{\boldsymbol{\delta}\}^{\perp}=-K_{\mu} \cap\{\boldsymbol{\delta}\}^{\perp}
\end{array}\right.
$$

where we have used propositions 26, 27 and 37. Expanding definition 35, this writes equivalently

$$
\left\{\begin{array}{c}
|\operatorname{dev} \boldsymbol{\delta}| \leqslant \frac{\operatorname{tr} \boldsymbol{\delta}}{\sqrt{N} \mu} \\
|\operatorname{dev} \boldsymbol{\tau}| \leqslant-\frac{\mu \operatorname{tr} \boldsymbol{\tau}}{\sqrt{N}}+\sigma_{y} \\
\boldsymbol{\delta}: \boldsymbol{\tau}=\frac{\sigma_{y}}{\sqrt{N} \mu} \operatorname{tr} \boldsymbol{\delta}
\end{array}\right.
$$

Let any $\boldsymbol{\xi} \in-T_{\mu, \sigma_{y}}$. From definition 40 of $T_{\mu, \sigma_{y}}$, we have

$$
|\operatorname{dev} \boldsymbol{\xi}| \leqslant-\frac{\mu \operatorname{tr} \boldsymbol{\xi}}{\sqrt{N}}+\sigma_{y}
$$


Then, expanding

$$
\begin{aligned}
(\boldsymbol{\xi}-\boldsymbol{\tau}): \boldsymbol{\delta}= & \boldsymbol{\xi}: \boldsymbol{\delta}-\frac{\sigma_{y}}{\sqrt{N} \mu} \operatorname{tr} \boldsymbol{\delta} \operatorname{from}(\mathrm{A} .16) \\
= & (\operatorname{dev} \boldsymbol{\xi}):(\operatorname{dev} \boldsymbol{\delta})+\frac{(\operatorname{tr} \boldsymbol{\xi})(\operatorname{tr} \boldsymbol{\delta})}{N}-\frac{\sigma_{y}}{\sqrt{N} \mu} \operatorname{tr} \boldsymbol{\delta} \\
\leqslant & |\operatorname{dev} \boldsymbol{\xi}||\operatorname{dev} \boldsymbol{\delta}|+\frac{(\operatorname{tr} \boldsymbol{\xi})(\operatorname{tr} \boldsymbol{\delta})}{N}-\frac{\sigma_{y}}{\sqrt{N} \mu} \operatorname{tr} \boldsymbol{\delta} \text { from the Cauchy-Schwartz inequality } \\
\leqslant & |\operatorname{dev} \boldsymbol{\xi}| \frac{\operatorname{tr} \boldsymbol{\delta}}{\sqrt{N} \mu}+\frac{(\operatorname{tr} \boldsymbol{\xi})(\operatorname{tr} \boldsymbol{\delta})}{N}-\frac{\sigma_{y}}{\sqrt{N} \mu} \operatorname{tr} \boldsymbol{\delta} \text { from (A.14) } \\
\leqslant & \left(-\frac{\mu \operatorname{tr} \boldsymbol{\xi}}{\sqrt{N}}+\sigma_{y}\right) \frac{\operatorname{tr} \boldsymbol{\delta}}{\sqrt{N} \mu}+\frac{(\operatorname{tr} \boldsymbol{\xi})(\operatorname{tr} \boldsymbol{\delta})}{N}-\frac{\sigma_{y}}{\sqrt{N} \mu} \operatorname{tr} \boldsymbol{\delta} \\
& \quad \text { from }(\mathrm{A} .17) \text { and since } \operatorname{tr} \boldsymbol{\delta} \geqslant 0 \text { from }(\mathrm{A} .14) \\
= & 0
\end{aligned}
$$

Thus $(\boldsymbol{\xi}-\boldsymbol{\tau}): \boldsymbol{\delta} \leqslant 0$ for all $\boldsymbol{\xi} \in-T_{\mu, \sigma_{y}}$ while (A.15) means that $\boldsymbol{\tau} \in-T_{\mu, \sigma_{y}}$. Recalling the definition 24 of the normal cone, this means that $\delta \in \mathscr{N}_{-T_{\mu, \sigma_{y}}}(\boldsymbol{\tau})$. From proposition 26, we have $\mathscr{N}_{-T_{\mu, \sigma_{y}}}=\partial \mathscr{I}_{-T_{\mu, \sigma_{y}}}$ and then $\boldsymbol{\delta} \in \partial \mathscr{I}_{-T_{\mu, \sigma_{y}}}(\boldsymbol{\tau})$ or, thanks to the Fenchel-Young theorem $4, \boldsymbol{\tau} \in \partial\left(\mathscr{I}_{-T_{\mu, \sigma_{y}}}\right)^{*}(\boldsymbol{\delta})$ which proves the backward inclusion and then completes the proof when $\mu \in] 0, \infty[$.

\section{Appendix A.8. Viscoplastic Drucker-Prager potential}

Proof: of theorem 2, page 9 .

Let $\boldsymbol{\delta} \in \partial \phi_{p}^{*}(\boldsymbol{\tau})$ or equivalently $\boldsymbol{\tau} \in \partial \phi_{p}(\boldsymbol{\delta})$, thanks to the the Fenchel-Young theorem 4. Then, from the definition (6e) of $\phi_{p}$, we have $\boldsymbol{\tau} \in 2 \eta \boldsymbol{\delta}+\partial\left(\mathscr{I}_{-T_{\mu, \sigma y}}\right)^{*}(\boldsymbol{\delta})$.

Assume first $\mu \in] 0, \infty\left[\right.$. Using proposition 41 , for expanding $\left(\mathscr{I}_{-T_{\mu, \sigma_{y}}}\right)^{*}$, we get

$$
\boldsymbol{\tau}-2 \eta \boldsymbol{\delta}-\frac{\sigma_{y}}{\sqrt{N} \mu} \boldsymbol{I} \in \partial \mathscr{I}_{K_{\frac{1}{\mu}}}(\boldsymbol{\delta})
$$

Next, let us turn to the expansion of $\partial \mathscr{I}_{K_{\frac{1}{\mu}}}$ by using proposition 39: the three cases of the disjunction are successively considered.

- sticking. Injecting (A.18a) in (A.8a) and rearranging, we get

$$
\left\{\begin{array}{l}
\boldsymbol{\delta}=0 \\
\sigma_{y}-\frac{\mu \operatorname{tr} \boldsymbol{\tau}}{\sqrt{N}} \geqslant|\operatorname{dev} \boldsymbol{\tau}|
\end{array}\right.
$$

From (A.18b), the subdifferential $\partial \phi_{p}^{*}(\boldsymbol{\tau})=\{0\}$. Since it contains exactly one element, $\phi_{p}^{*}$ is differentiable in that case and $\nabla \phi_{p}^{*}(\boldsymbol{\tau})=0$. Let us check that the right-hand-side of (6f) coincides with the expected result. Using (A.18c) we obtain $\sigma_{y}-\frac{\mu \operatorname{tr} \boldsymbol{\tau}}{\sqrt{N}} \geqslant|\operatorname{dev} \boldsymbol{\tau}| \geqslant 0 \geqslant-\mu^{2}|\operatorname{dev} \boldsymbol{\tau}|$ and then $(6 \mathrm{~g})$ gives $\kappa_{\mu, \sigma_{c}}(\boldsymbol{\tau})=0$. Finally (6f) leads to $\nabla \phi_{p}^{*}(\boldsymbol{\tau})=0$ which is the expected result since $\boldsymbol{\delta}=0$.

- sliding. Injecting (A.18a) in (A.8b), we get, after rearrangements

$$
\left\{\begin{array}{l}
\operatorname{dev} \boldsymbol{\tau}=\left\{2 \eta+\frac{1}{|\operatorname{dev} \boldsymbol{\delta}|}\left(\sigma_{y}-\frac{\mu}{\sqrt{N}} \operatorname{tr}(\boldsymbol{\tau}-2 \eta \boldsymbol{\delta})\right)\right\} \operatorname{dev} \boldsymbol{\delta} \\
\operatorname{tr} \boldsymbol{\delta}=\sqrt{N} \mu|\operatorname{dev} \boldsymbol{\delta}| \neq 0 \\
\sigma_{y}-\frac{\mu}{\sqrt{N}} \operatorname{tr}(\boldsymbol{\tau}-2 \eta \boldsymbol{\delta}) \geqslant 0
\end{array}\right.
$$


Let us take the norm of (A.18d). Using (A.18f) for solving the sign, we obtain successively

$$
\begin{aligned}
|\operatorname{dev} \boldsymbol{\tau}| & =2 \eta|\operatorname{dev} \boldsymbol{\delta}|+\sigma_{y}-\frac{\mu \operatorname{tr}(\boldsymbol{\tau}-2 \eta \boldsymbol{\delta})}{\sqrt{N}} \\
& =2 \eta\left(1+\mu^{2}\right)|\operatorname{dev} \boldsymbol{\delta}|+\sigma_{y}-\frac{\mu \operatorname{tr} \boldsymbol{\tau}}{\sqrt{N}} \text { from (A.18e) } \\
\Longleftrightarrow|\operatorname{dev} \boldsymbol{\delta}| & =\frac{1}{2 \eta\left(1+\mu^{2}\right)}\left(|\operatorname{dev} \boldsymbol{\tau}|-\sigma_{y}+\frac{\mu \operatorname{tr} \boldsymbol{\tau}}{\sqrt{N}}\right)
\end{aligned}
$$

Note that (A.18e) leads to $|\operatorname{dev} \boldsymbol{\delta}|>0$ that also expresses as a condition upon $\boldsymbol{\tau}$ only as

$$
\sigma_{y}-\frac{\mu \operatorname{tr} \boldsymbol{\tau}}{\sqrt{N}}<|\operatorname{dev} \boldsymbol{\tau}|
$$

Replacing the previous expression of $|\operatorname{dev} \boldsymbol{\delta}|$ in (A.18e), we get

$$
\operatorname{tr} \boldsymbol{\delta}=\frac{\sqrt{N} \mu}{2 \eta\left(1+\mu^{2}\right)}\left(|\operatorname{dev} \boldsymbol{\tau}|-\sigma_{y}+\frac{\mu \operatorname{tr} \boldsymbol{\tau}}{\sqrt{N}}\right)
$$

The condition (A.18f) could now be expressed in terms of $\boldsymbol{\tau}$ only by replacing the previous expression of $\operatorname{tr} \boldsymbol{\delta}$. After rearrangements, we get

$$
\sigma_{y}-\frac{\mu \operatorname{tr} \boldsymbol{\tau}}{\sqrt{N}} \geqslant-\mu^{2}|\operatorname{dev} \boldsymbol{\tau}|
$$

Note that satisfying together (A.18g) and (A.18h) implies $|\operatorname{dev} \boldsymbol{\tau}| \neq 0$. Observe from (A.18d) that $\operatorname{dev} \boldsymbol{\tau}$ and $\operatorname{dev} \boldsymbol{\delta}$ are two collinear tensors and then

$$
\operatorname{dev} \boldsymbol{\delta}=|\operatorname{dev} \boldsymbol{\delta}| \frac{\operatorname{dev} \boldsymbol{\tau}}{|\operatorname{dev} \boldsymbol{\tau}|}=\frac{1}{2 \eta\left(1+\mu^{2}\right)}\left(|\operatorname{dev} \boldsymbol{\tau}|-\sigma_{y}+\frac{\mu \operatorname{tr} \boldsymbol{\tau}}{\sqrt{N}}\right) \frac{\operatorname{dev} \boldsymbol{\tau}}{|\operatorname{dev} \boldsymbol{\tau}|}
$$

Grouping the two previous expressions yields

$$
\boldsymbol{\delta}=\operatorname{dev} \boldsymbol{\delta}+\frac{\operatorname{tr} \boldsymbol{\delta}}{N} \boldsymbol{I}=\frac{1}{2 \eta\left(1+\mu^{2}\right)}\left(|\operatorname{dev} \boldsymbol{\tau}|-\sigma_{y}+\frac{\mu \operatorname{tr} \boldsymbol{\tau}}{\sqrt{N}}\right)\left(\frac{\operatorname{dev} \boldsymbol{\tau}}{|\operatorname{dev} \boldsymbol{\tau}|}+\frac{\mu}{\sqrt{N}} \boldsymbol{I}\right)
$$

Thus, the subdifferential $\partial \phi_{p}^{*}(\boldsymbol{\tau})$ contains exactly one element, i.e. $\phi_{p}^{*}$ is differentiable in the sliding case also. Let us check that the right-hand-side of (6f) coincides with the expected result. Using the inequalities (A.18g) and (A.18h), we obtain successively from (6g)-(6h) that $\xi_{\mu, \sigma_{y}}(\boldsymbol{\tau})=\frac{\mu \operatorname{tr} \boldsymbol{\tau}}{\sqrt{N}}-\mu^{2}|\operatorname{dev} \boldsymbol{\tau}|$ and $\kappa_{\mu, \sigma_{y}}(\boldsymbol{\tau})=\left(|\operatorname{dev} \boldsymbol{\tau}|-\sigma_{y}+\frac{\mu \operatorname{tr} \boldsymbol{\tau}}{\sqrt{N}}\right) /|\operatorname{dev} \boldsymbol{\tau}|>0$. Finally (6f) furnishes an expression of $\nabla \phi_{p}^{*}(\boldsymbol{\tau})$ that coincides with (A.18i).

- losing contact. Injecting (A.18a) in (A.8c), we get

$$
\left\{\begin{array}{l}
\boldsymbol{\tau}-2 \eta \boldsymbol{\delta}-\frac{\sigma_{y}}{\sqrt{N} \mu} \boldsymbol{I}=0 \\
|\operatorname{dev} \boldsymbol{\delta}|<\frac{\operatorname{tr} \boldsymbol{\delta}}{\sqrt{N} \mu}
\end{array}\right.
$$

and (A.18j) yields

$$
\boldsymbol{\delta}=\frac{1}{2 \eta}\left(\boldsymbol{\tau}-\frac{\sigma_{y}}{\sqrt{N} \mu} \boldsymbol{I}\right)
$$

Thus, $\partial \phi_{p}^{*}(\boldsymbol{\sigma})$ contains exactly one element and $\phi_{p}^{*}$ is differentiable. The condition (A.18k) expresses equivalently in terms of $\boldsymbol{\tau}$ as

$$
\sigma_{y}-\frac{\mu \operatorname{tr} \boldsymbol{\tau}}{\sqrt{N}}<-\mu^{2}|\operatorname{dev} \boldsymbol{\tau}|
$$


Let us check again that the right-hand-side of (6f) coincides with the expected result. Using $\left(\right.$ A.18m), we obtain from $(6 \mathrm{~g})-(6 \mathrm{~h})$ that $\xi_{\mu, \sigma_{y}}(\boldsymbol{\tau})=\sigma_{y}$ and $\kappa_{\mu, \sigma_{y}}(\boldsymbol{\tau})=1+\mu^{2}$. Finally (6f) furnishes an expression of $\nabla \phi_{p}^{*}(\boldsymbol{\tau})$ that coincides with (A.18i).

For all these three cases, $\partial \phi_{p}^{*}(\boldsymbol{\tau})$ contains exactly one element and thus $\phi_{p}^{*}$ is globally differentiable. The elevation view on Fig. 3.bottom-left shows that $\kappa_{\mu, \sigma_{y}}$ is continuous except at the junction between the three cones and is differentiable except along the cone boundaries. At this junction, the second factor in (6f) vanishes and finally, $\nabla \phi_{p}^{*}$ is continuous everywhere.

\section{Appendix B. Numerical resolution}

\section{Appendix B.1. Problem simplification}

Before its numerical resolution, the full problem (5a)-(5d) is simplified as:

$(Q)$ : find the elastic deformation $\boldsymbol{\gamma}_{e}$, the damage $d$ and the velocity $\boldsymbol{u}$ satisfying

$$
\left\{\begin{aligned}
\frac{\partial \boldsymbol{\gamma}_{e}}{\partial t}+ & \nabla \phi_{p}^{*}\left([d] ; \mathbb{A}(d) \boldsymbol{\gamma}_{e}\right)-D(\boldsymbol{u})=0 \\
\frac{\partial d}{\partial t}= & \nabla \phi_{d}^{*}\left(\left[\boldsymbol{\gamma}_{e}, d\right] ;\left\{-\mathbb{A}^{\prime}(d) \boldsymbol{\gamma}_{e}\right\}: \boldsymbol{\gamma}_{e}\right) \\
& -\operatorname{div}\left(2 \eta_{s} D(\boldsymbol{u})+\mathbb{A}(d) \boldsymbol{\gamma}_{e}\right)=\boldsymbol{f}
\end{aligned}\right.
$$

Note that, in (B.1a), the upper-convected tensor derivative is replaced by a simple time derivative. Correspondingly, in (B.1b), the Lagrange derivative of the damage is also replaced by a simple time derivative. In the conservation of momentum (B.1c), the inertia terms are neglected, since only slow flows are considered here. Finally, the original incompressibility constraint (5d) is not considered here and the corresponding Lagrange multiplier, the pressure $p$, disappears in (B.1c). All these simplifications are very classical when only small deformations are considered, which is the case for the uniaxial compression benchmark considered here. The system (B.1a)-(B.1c) is closed by suitable initial and boundary conditions.

Such simplifications allow us to focus on the interplay between the main nonlinearities introduced by the two dissipation potentials $\phi_{p}$ and $\phi_{d}$, expressed by (6e) and (6k), respectively, while the elasticity operator $\mathbb{A}$ is given by (6a). Note that the numerical procedure developed in this appendix could be easily adapted to others choices of the dissipation potentials and to the reintroduction of incompressibility and full time derivatives. While the final numerical algorithm is relatively simple, its correct derivation requires some technical computations.

\section{Appendix B.2. Dimensionless procedure}

Let $L$ and $U$ be characteristic length and velocity, respectively. The characteristic time is $L / U$ and the characteristic stress is $\left(\eta_{s}+\eta_{0}\right) U / L$. The dimensionless variables and unknown are denoted with tildes and defined by

$$
\tilde{x}=\frac{x}{L}, \quad \tilde{t}=\frac{U t}{L}, \quad \tilde{\boldsymbol{u}}=\frac{\boldsymbol{u}}{U}, \quad \tilde{\boldsymbol{\sigma}}=\frac{L \boldsymbol{\sigma}}{\left(\eta_{s}+\eta_{0}\right) U}, \quad \tilde{w}=\frac{L^{2}}{\left(\eta_{s}+\eta_{0}\right) U^{2}} w
$$

while $d$ and $\gamma_{e}$, which are already dimensionless, are unchanged. In the rest of this appendix, only dimensionless variables are considered. Thus, for simplicity and since there is no ambiguity, tildes are omitted on the dimensionless variables.

Recall that $E, \eta$ and $\sigma_{y}$ are defined by (6c), (6i) and (6j) respectively, and all of them present a singular factor $1-d$. Thus, in expression(6f) of $\nabla \phi_{p}^{*}$, both the numerator and the denominator involve this $1-d$ factor that is zero at the limit of a fully damaged material. The expression of $\nabla \phi_{p}^{*}$ could be nicely extended by continuity at this limit: for this, all expressions, both in 
the numerator and the denominator, are divided by $1-d$, and the corresponding coefficients are denoted with a hat. Relation (3d) writes in dimensionless form:

$$
\begin{array}{ll} 
& \partial_{t} \boldsymbol{\gamma}_{e}+\frac{\kappa_{\mu, \gamma_{y}}\left(\hat{\boldsymbol{\sigma}}_{e}\right)}{2 \alpha W e\left(1+\mu^{2}\right)}\left(\hat{\boldsymbol{\sigma}}_{e}-\frac{\xi_{\mu, \gamma_{y}}\left(\hat{\boldsymbol{\sigma}}_{e}\right)}{\sqrt{N} \mu} \boldsymbol{I}\right)=D(\boldsymbol{u}) \\
\text { where } \quad & \hat{\boldsymbol{\sigma}}_{e}=\widehat{\mathbb{A}}(d) \boldsymbol{\gamma}_{e}=2 \widehat{G}(d) \boldsymbol{\gamma}_{e}+\hat{\lambda}(d)\left(\operatorname{tr} \boldsymbol{\gamma}_{e}\right) \boldsymbol{I} \\
& \hat{\lambda}(d)=\frac{\nu(d)}{(1+\nu(d))(1-2 \nu(d))} \text { and } \hat{G}(d)=\frac{1}{2(1+\nu(d))}
\end{array}
$$

Recall that, from (6d), the Poisson ratio writes $\nu(d)=\nu_{0}+\left(\nu_{1}-\nu_{0}\right) d$. The dimensionless numbers involved in the previous equations are given by

$$
W e=\frac{U\left(\eta_{s}+\eta_{0}\right)}{L E_{0}} \text { and } \gamma_{y}=\frac{\sigma_{y 0}}{E_{0}}, \quad \alpha=\frac{\eta_{0}}{\eta_{s}+\eta_{0}}
$$

Here, $W e$ is the Weissenberg number, $\gamma_{y}$ is a plastic yield deformation and $\alpha$ is a viscosity ratio. Note that the usual Bingham number, defined by $B i=L \sigma_{y 0} /\left(U\left(\eta_{s}+\eta_{0}\right)\right)$ is such that $\gamma_{y}=B i W e$. Relation (3e) writes in dimensionless form:

$$
\frac{\partial d}{\partial t}=\frac{(1-d) \kappa_{\mu, \gamma_{c}}\left(\hat{\boldsymbol{\sigma}}_{e}\right)}{2 W e_{d}\left(1+\mu^{2}\right)}\left(-\mathbb{A}^{\prime}(d) \boldsymbol{\gamma}_{e}\right): \boldsymbol{\gamma}_{e}
$$

with the dimensionless elasticity operator $\mathbb{A}(d)=(1-1) \widehat{\mathbb{A}}(d)$ and where the dimensionless numbers are defined by

$$
W e_{d}=\frac{U \eta_{d}}{L E_{0}} \text { and } \gamma_{c}=\frac{\sigma_{c}}{E_{0}}
$$

Here, $W e_{d}$ is a damage-related Weissenberg number, and $\gamma_{c}$ is a damage yield deformation. Conversely, by introducing a damage related Bingham dimensionless number $B i_{d}=L \sigma_{c} /\left(\eta_{d} U\right)$, we have $\gamma_{c}=W e_{d} B i_{d}$. The dimensionless Cauchy stress writes:

$$
\boldsymbol{\sigma}=2(1-\alpha) D(\boldsymbol{u})+\frac{(1-d)}{W e} \widehat{\boldsymbol{\sigma}}_{e}
$$

and the dimensionless dissipation writes

$$
\begin{aligned}
& w_{p}=2(1-\alpha)|D(\boldsymbol{u})|^{2}+\frac{(1-d)}{\alpha W e^{2}} \frac{\kappa_{\mu, \gamma_{y}}\left(\hat{\boldsymbol{\sigma}}_{e}\right)}{2\left(1+\mu^{2}\right)} \hat{\boldsymbol{\sigma}}_{e}:\left(\hat{\boldsymbol{\sigma}}_{e}-\frac{\xi_{\mu, \gamma_{y}}\left(\hat{\boldsymbol{\sigma}}_{e}\right)}{\sqrt{N \mu} \boldsymbol{I})}\right. \\
& w_{d}=\frac{(1-d)}{W e W e_{d}} \frac{\kappa_{\mu, \gamma_{c}}\left(\hat{\boldsymbol{\sigma}}_{e}\right)}{2\left(1+\mu^{2}\right)}\left\{\left(-\mathbb{A}^{\prime}(d) \boldsymbol{\gamma}_{e}\right): \boldsymbol{\gamma}_{e}\right\}^{2}
\end{aligned}
$$

Finally, the present model contains eight independent dimensionless numbers: $W e, W e_{d}, \gamma_{y} \gamma_{c}$, $\mu, \nu_{0}, \nu_{1}$ and $\alpha$.

\section{Appendix B.3. Implicit time discretization and fixed-point algorithm}

Let $\Delta t>0$ be the dimensionless time step and $t_{n}=n \Delta t, n \geqslant 0$. The two constitutive equations are discretized with respect to time by using a fully implicit first order scheme. At time step $t_{n}$, $n \geqslant 1$, assume that $\gamma_{e, n-1}, \mathbf{u}_{n-1}$ and $d_{n-1}$ are known. Then, the first order time discretization of the problem leads to compute $\gamma_{e, n}, d_{n}$ and $\mathbf{u}_{n}$ by a fixed-point inner loop for solving the nonlinearities. Let $k$ denotes the index of this inner loop. The fixed point algorithm writes:

- When $k=0$, let $\left(\gamma_{e, n, 0}, d_{n, 0}, \boldsymbol{u}_{n, 0}\right)=\left(\gamma_{e, n-1}, d_{n-1}, \boldsymbol{u}_{n-1}\right)$ 
- When $k \geqslant 1$, assume that $\left(\gamma_{e, n, k-1}, d_{n, k-1}, \mathbf{u}_{n, k-1}\right)$ are known.

step 1: find $\gamma_{e, n, k}$ and $\boldsymbol{u}_{n, k}$ such that

$$
\left\{\begin{array}{l}
\frac{\boldsymbol{\gamma}_{e, n, k}-\boldsymbol{\gamma}_{e, n-1}}{\Delta t}+\mathbb{V}\left(d_{n, k-1}, \boldsymbol{\gamma}_{e, n, k-1} ; \boldsymbol{\gamma}_{e, n, k}\right)-D\left(\boldsymbol{u}_{n, k}\right)=0 \\
-\operatorname{div}\left(2(1-\alpha) D\left(\boldsymbol{u}_{n, k}\right)+\frac{(1-d)}{W e} \widehat{\mathbb{A}}\left(d_{n, k-1}\right) \boldsymbol{\gamma}_{e, n, k}\right)=\boldsymbol{f}\left(t_{n}\right) \\
\boldsymbol{u}_{n, k}=\boldsymbol{u}_{\Gamma}\left(t_{n}\right) \text { on } \partial \Omega
\end{array}\right.
$$

step 2: compute explicitly

$$
\begin{aligned}
Y_{n, k-1} & =\left(-\mathbb{A}^{\prime}\left(d_{n, k-1}\right) \gamma_{e, n, k}\right): \gamma_{e, n, k} \\
\breve{d}_{n, k} & =d_{n-1}+\frac{\Delta t\left(1-d_{n, k-1}\right) \kappa_{\mu, \gamma_{c}}\left(\widehat{\mathbb{A}}\left(d_{n, k-1}\right) \gamma_{e, n, k}\right)}{2 W e_{d}\left(1+\mu^{2}\right)} Y_{n, k-1} \\
d_{n, k} & =\max \left(0, \min \left(1, \breve{d}_{n, k}\right)\right)
\end{aligned}
$$

- The fixed point loop stops when the residual terms drops below a prescribed tolerance and then we set $\left(\gamma_{e, n}, d_{n}, \mathbf{u}_{n}\right)=\left(\gamma_{e, n, k}, d_{n, k}, \mathbf{u}_{n}\right)$.

In (B.2a), the notation $\mathbb{V}\left(d, \gamma_{e, n, k-1} ; \boldsymbol{\gamma}_{e, n, k}\right)$ stands for

$$
\begin{aligned}
\mathbb{V}\left(d_{n, k-1}, \gamma_{e, n, k-1} ; \gamma_{e, n, k}\right) & =\frac{\widehat{\kappa}_{n, k-1}}{2 \alpha W e\left(1+\mu^{2}\right)}\left(\widehat{\mathbb{A}}\left(d_{n, k-1}\right) \boldsymbol{\gamma}_{e, n, k}-\frac{\widehat{\xi}_{n, k-1}}{\sqrt{N} \mu} \boldsymbol{I}\right) \\
\text { where } \quad \widehat{\kappa}_{n, k-1} & =\kappa_{\mu, \gamma_{y}}\left(\widehat{\mathbb{A}}\left(d_{n, k-1}\right) \boldsymbol{\gamma}_{e, n, k-1}\right) \\
\widehat{\xi}_{n, k-1} & =\xi_{\mu, \gamma_{y}}\left(\widehat{\mathbb{A}}\left(d_{n, k-1}\right) \gamma_{e, n, k-1}\right)
\end{aligned}
$$

Observe that $\mathbb{V}(., . ;$.$) is affine with respect to its last variable: it represents a rearrangement of$ $\nabla \phi_{p}^{*}\left([d], \mathbb{A}(d) \boldsymbol{\gamma}_{e, n, k}\right)$ by re-balancing the evaluation of terms from the $k-1$ and $k$ iterations of the fixed point. Note that, after this splitting between $\gamma_{e, n, k-1}$ and $\gamma_{e, n, k}$, the consistency of the fixed point is still satisfied, i.e.:

$$
\mathbb{V}\left(d, \gamma_{e} ; \gamma_{e}\right)=\nabla \phi_{p}^{*}\left([d] ; \gamma_{e}\right), \quad \forall d \in[0,1] \text { and } \gamma_{e} \in \mathbb{R}_{s}^{N \times N}
$$

Observe that the first subproblem (B.2a)-(B.2c) is now linear: the two unknowns $\gamma_{e, n, k}$ and $\boldsymbol{u}_{n, k}$ are highlighted in blue for clarity. The second one (B.2d)-(B.2e) is now explicit. An additional projection on $[0,1]$ has been introduced, since, after time discretization, there is no more guaranty for $\breve{d}_{n, k}$ to belongs in $[0,1]$.

The constitutive (B.2a) and momentum (B.2b) equations are solved simultaneously for the elastic deformation $\gamma_{e, n, k}$ and the velocity $\boldsymbol{u}_{n, k}$, using the known damage $d_{n, k-1}$. Then, using (B.2d)(B.2f), the damage is re-evaluated as $d_{n, k}$, based on this value of $\gamma_{e, n, k}$.

The linear subproblem (B.2a)-(B.2c) is solved in two steps. First, (B.2a) writes equivalently as

$$
\left(1+2 \Delta t \breve{G}_{n, k-1}\right) \gamma_{e, n, k}+\Delta t\left(\check{\lambda}_{n, k-1}\left(\operatorname{tr} \gamma_{e, n, k}\right)-\check{\xi}_{n, k-1}\right) \boldsymbol{I}=\gamma_{e, n-1}+\Delta t D\left(\boldsymbol{u}_{n, k}\right)
$$

where $\quad \breve{G}_{n, k-1}=c_{n, k-1} \widehat{G}\left(d_{n, k-1}\right), \quad \check{\lambda}_{n, k-1}=c_{n, k-1} \hat{\lambda}\left(d_{n, k-1}\right), \quad \check{\xi}_{n, k-1}=c_{n, k-1} \frac{\widehat{\xi}_{n, k-1}}{\sqrt{N} \mu}$

and $\quad c_{n, k-1}=\frac{\widehat{\kappa}_{n, k-1}}{2 \alpha W e\left(1+\mu^{2}\right)}$ for convenience. 
Tacking the deviatoric and trace parts of the previous equation, it explicitly solves as

$$
\begin{aligned}
\operatorname{dev} \gamma_{e, n, k} & =a_{n, k-1} \operatorname{dev}\left(\boldsymbol{\gamma}_{e, n-1}+\Delta t D\left(\boldsymbol{u}_{n, k}\right)\right) \\
\operatorname{tr} \gamma_{e, n, k} & =b_{n, k-1}\left\{\operatorname{tr}\left(\gamma_{e, n-1}+\Delta t D\left(\boldsymbol{u}_{n, k}\right)\right)+N \Delta t \check{\xi}_{n, k-1}\right\} \\
\text { with } \quad a_{n, k-1} & =\left(1+2 \Delta t \check{G}_{n, k-1}\right)^{-1} \\
b_{n, k-1} & =\left(1+2 \Delta t\left(\check{G}_{n, k-1}+\frac{N}{2} \check{\lambda}_{n, k-1}\right)\right)^{-1} \\
\text { and then } \quad \gamma_{e, n, k} & =\operatorname{dev} \gamma_{e, n, k}+\left(\operatorname{tr} \gamma_{e, n, k}\right) \frac{\boldsymbol{I}}{N} \\
& =a_{n, k-1} \operatorname{dev}\left(\gamma_{e, n-1}+\Delta t D\left(\boldsymbol{u}_{n, k}\right)\right) \\
& +b_{n, k-1}\left\{\operatorname{tr}\left(\boldsymbol{\gamma}_{e, n-1}+\Delta t D\left(\boldsymbol{u}_{n, k}\right)\right)+N \Delta t \check{\xi}_{n, k-1}\right\} \frac{\boldsymbol{I}}{N}
\end{aligned}
$$

This explicit expression of $\gamma_{e, n, k}$ in terms of the unknown velocity $\boldsymbol{u}_{n, k}$ is then replaced in (B.2b), in order to obtain a problem for $\boldsymbol{u}_{n, k}$ only:

(S): find $\boldsymbol{u}_{n, k}$ such that

$$
\left\{\begin{aligned}
-\operatorname{div}\left(2 \eta_{1, n, k-1} \operatorname{dev} D\left(\boldsymbol{u}_{n, k}\right)+2 \eta_{2, n, k-1}\left(\operatorname{div} \boldsymbol{u}_{n, k}\right) \frac{\boldsymbol{I}}{N}\right) & =\boldsymbol{f}\left(t_{n}\right)+\operatorname{div} \boldsymbol{\chi}_{n, k-1} \\
\boldsymbol{u}_{n, k} & =\boldsymbol{u}_{\Gamma}\left(t_{n}\right) \text { on } \partial \Omega
\end{aligned}\right.
$$

where

$$
\begin{aligned}
& \eta_{1, n, k-1}= 1-\alpha+\frac{\left(1-d_{n, k-1}\right) \Delta t}{W e} a_{n, k-1} \widehat{G}\left(d_{n, k-1}\right) \\
& \eta_{2, n, k-1}=1-\alpha+\frac{\left(1-d_{n, k-1}\right) \Delta t}{W e} b_{n, k-1}\left(\widehat{G}\left(d_{n, k-1}\right)+\frac{N}{2} \widehat{\lambda}\left(d_{n, k-1}\right)\right) \\
& \chi_{n, k-1}=\frac{2\left(1-d_{n, k-1}\right)}{W e}\left\{\begin{array}{l}
a_{n, k-1} \widehat{G}\left(d_{n, k-1}\right) \operatorname{dev} \gamma_{e, n-1} \\
\left.+b_{n, k-1}\left(\widehat{G}\left(d_{n, k-1}\right)+\frac{N}{2} \widehat{\lambda}\left(d_{n, k-1}\right)\right)\left(\operatorname{tr} \gamma_{e, n-1}+N \Delta t \check{\xi}_{n, k-1}\right) \frac{\boldsymbol{I}}{N}\right\}
\end{array}\right.
\end{aligned}
$$

Remark that $\chi_{n, k-1}$ depends upon both $\operatorname{tr} \gamma_{e, n-1}$ and $\operatorname{tr} \gamma_{e, n, k-1}$ via $a_{n, k-1}$ and $b_{n, k-1}$. Assuming $\alpha<1$, observe that $\eta_{1, n, k-1}$ and $\eta_{2, n, k-1}$ are always strictly positive, even in the full damaged case $d_{n, k-1}=1$. Then, subproblem (B.5a)-(B.5b) is always well-posed: this elliptic system can be solved by completely standard methods (see e.g. [52, chap 2]). After this resolution, $\boldsymbol{u}_{n, k}$ is known and then $\gamma_{e, n, k}$ can be computed explicitly from (B.4). Finally, the right-hand-side of (B.2f) can therefore be evaluated: this leads to an explicit computation for $d_{n, k}$ that solves the second step of the fixed point loop.

\section{Appendix B.4. Space discretization}

Let us turn to the numerical resolution of subproblem (B.5a)-(B.5b). Consider the following bilinear form $a$ and linear form $\ell$, defined for all $\boldsymbol{u}, \boldsymbol{v} \in\left(H^{1}(\Omega)\right)^{N}$ by:

$$
\begin{aligned}
a_{n, k-1}(\boldsymbol{u}, \boldsymbol{v}) & =\int_{\Omega}\left(2 \eta_{1, n, k-1} \operatorname{dev}(D(\boldsymbol{u})): \operatorname{dev}(D(\boldsymbol{v}))+\frac{2 \eta_{2, n, k-1}}{N} \operatorname{div}(\boldsymbol{u}) \operatorname{div}(\boldsymbol{v})\right) \mathrm{d} \boldsymbol{x} \\
\ell_{n, k-1}(\boldsymbol{v}) & =\int_{\Omega}\left(\boldsymbol{f}\left(t_{n}\right) \cdot \boldsymbol{v}-\boldsymbol{\chi}_{n, k-1}: D(\boldsymbol{v})\right) \mathrm{d} \boldsymbol{x}
\end{aligned}
$$


For all $\boldsymbol{u}_{b} \in\left(H^{\frac{1}{2}}(\partial \Omega)\right)^{N}$, we also introduce the following function space:

$$
V\left(\boldsymbol{u}_{b}\right)=\left\{\boldsymbol{v} \in\left(H^{1}(\Omega)\right)^{N} ; \boldsymbol{v}=\boldsymbol{u}_{b} \text { on } \partial \Omega\right\}
$$

Then, the variational formulation of (B.5a)-(B.5b) writes: find $\boldsymbol{u}_{n, k} \in V\left(\boldsymbol{u}_{\Gamma}\left(t_{n}\right)\right)$ such that

$$
a_{n, k-1}\left(\boldsymbol{u}_{n, k}, \boldsymbol{v}\right)=\ell_{n, k-1}(\boldsymbol{v}), \quad \forall \boldsymbol{v} \in V(0)
$$

The space for the velocities $\left(H^{1}(\Omega)\right)^{N}$ is approximated by piecewise linear and continuous functions on a finite element mesh of the flow domain $\Omega$. Conversely, the elastic deformation $\gamma_{e}$ and the damage $d$ are approximated by piecewise constant functions. The numerical resolution is implemented by using the Rheolef finite element library [52]. 